\title{
Spinors of real type as polyforms and the generalized Killing equation
}

\author{
Vicente Cortés $^{1} \cdot$ Calin Lazaroiu $^{2,3} \cdot$ C. S. Shahbazi ${ }^{1}$
}

Received: 8 March 2020 / Accepted: 3 February 2021 / Published online: 19 March 2021

(c) The Author(s) 2021

\begin{abstract}
We develop a new framework for the study of generalized Killing spinors, where every generalized Killing spinor equation, possibly with constraints, can be formulated equivalently as a system of partial differential equations for a polyform satisfying algebraic relations in the Kähler-Atiyah bundle constructed by quantizing the exterior algebra bundle of the underlying manifold. At the core of this framework lies the characterization, which we develop in detail, of the image of the spinor squaring map of an irreducible Clifford module $\Sigma$ of real type as a real algebraic variety in the Kähler-Atiyah algebra, which gives necessary and sufficient conditions for a polyform to be the square of a real spinor. We apply these results to Lorentzian four-manifolds, obtaining a new description of a real spinor on such a manifold through a certain distribution of parabolic 2-planes in its cotangent bundle. We use this result to give global characterizations of real Killing spinors on Lorentzian four-manifolds and of fourdimensional supersymmetric configurations of heterotic supergravity. In particular, we find new families of Einstein and non-Einstein four-dimensional Lorentzian metrics admitting real Killing spinors, some of which are deformations of the metric of $\mathrm{AdS}_{4}$ space-time.
\end{abstract}

Keywords Spin geometry · Generalized Killing spinors · Spinor bundles · Lorentzian geometry

Mathematics Subject Classification Primary 53C27; Secondary 53C50

\footnotetext{
C. S. Shahbazi

carlos.shahbazi@uni-hamburg.de

Vicente Cortés

vicente.cortes@uni-hamburg.de

Calin Lazaroiu

lcalin@theory.nipne.ro

1 Department of Mathematics, University of Hamburg, Hamburg, Germany

2 Center for Geometry and Physics, Institute for Basic Science, Pohang, Republic of Korea

3 Department of Theoretical Physics, Horia Hulubei National Institute for Physics and Nuclear Engineering, Bucharest, Romania
} 


\section{Contents}

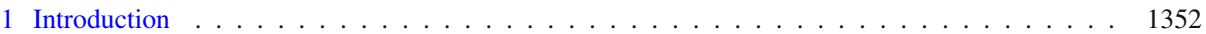

1.1 Background and context . . . . . . . . . . . . . . . . . . . . . 1352

1.2 Main results . . . . . . . . . . . . . . . . . . . . . . . . . . . . 1354

1.3 Open problems and further directions . . . . . . . . . . . . . . . . . . . . . . . . . . . . . . . . . . . . . . . . . . . . . . . . . . . . . . . . . . . .

1.4 Outline of the paper . . . . . . . . . . . . . . . . . . . . . . . . . 1357

2 Representing real vectors as endomorphisms in a paired vector space . . . . . . . . . . . . . . 1358

2.1 Tame endomorphisms and the squaring maps . . . . . . . . . . . . . . . . . . . . . . 1359

2.2 Admissible endomorphisms . . . . . . . . . . . . . . . . . . . . . . . . . . . . . . . . . . . . . . . . . . . . . . . . . . . . . . . . . . .

2.3 The manifold $\dot{\mathcal{Z}}$ and the projective squaring map . . . . . . . . . . . . . . . . . . 1361

2.4 Tamings of $\mathscr{B} \ldots \ldots \ldots \ldots \ldots \ldots \ldots \ldots$

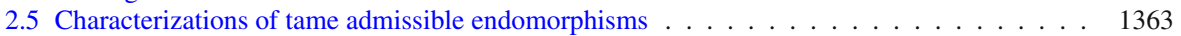

2.6 Two-dimensional examples . . . . . . . . . . . . . . . . . . . . . . . . . . . 1366

2.7 Including linear constraints . . . . . . . . . . . . . . . . . . . . . . . . . . . . . . 1368

3 From real spinors to polyforms . . . . . . . . . . . . . . . . . . . . . . . . . . . . 1368

3.1 Admissible pairings for irreducible real Clifford modules . . . . . . . . . . . . . . . . . . 1368

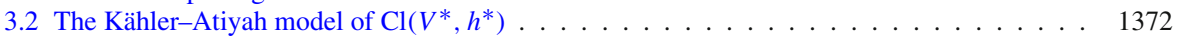

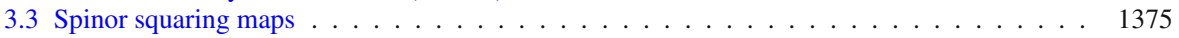

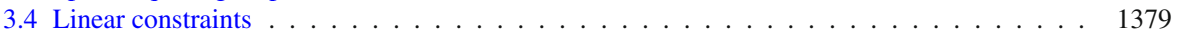

3.5 Real chiral spinors . . . . . . . . . . . . . . . . . . . . . . . . . . . . . . . . 1379

3.6 Low-dimensional examples . . . . . . . . . . . . . . . . . . . . . . . . . . . . . . . . . . . 1380

3.6 .1 Signature $(2,0) \ldots \ldots \ldots \ldots \ldots \ldots \ldots$

3.6 .2 Signature $(1,1) \ldots \ldots \ldots \ldots \ldots \ldots \ldots \ldots \ldots$

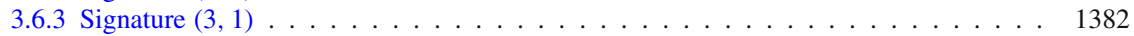

3.6 .4 Signature $(2,2) \ldots \ldots \ldots \ldots \ldots \ldots \ldots \ldots$

4 Constrained generalized Killing spinors of real type . . . . . . . . . . . . . . . . . . . 1386

4.1 Bundles of real simple Clifford modules . . . . . . . . . . . . . . . . . . . . . . . . . . . 1387

4.2 Paired spinor bundles . . . . . . . . . . . . . . . . . . . . . . . . . . . . . . . . . . . . 1389

4.3 Constrained generalized Killing spinors . . . . . . . . . . . . . . . . . . . . . . . . . . . 1391

4.4 Spinor squaring maps . . . . . . . . . . . . . . . . . . . . . . . . . . . . . . 1391

4.5 Description of constrained generalized Killing spinors as polyforms . . . . . . . . . . . . . . 1394

4.6 Real spinors on Lorentzian four-manifolds . . . . . . . . . . . . . . . . . . . . . . . . . . . 1396

4.7 Real spinors on globally hyperbolic Lorentzian four-manifolds . . . . . . . . . . . . . . . . 1397

5 Real Killing spinors on Lorentzian four-manifolds . . . . . . . . . . . . . . . . . . . . . . . . . 1399

5.1 Describing real Killing spinors through differential forms . . . . . . . . . . . . . . . . . . . 1400

5.2 The Pfaffian system and its consequences . . . . . . . . . . . . . . . . . . . . . . . 1401

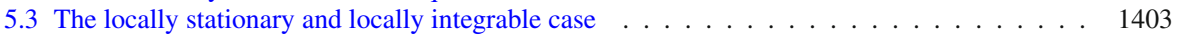

5.4 Special solutions from the Poincaré half-plane . . . . . . . . . . . . . . . . . . . . . . . 1406

6 Supersymmetric heterotic configurations . . . . . . . . . . . . . . . . . . . . . . . . . . . . . 1409

6.1 Supersymmetric heterotic configurations . . . . . . . . . . . . . . . . . . . . . . . . 1409

6.2 Characterizing supersymmetric heterotic configurations through differential forms . . . . . . 1410

6.3 Some examples . . . . . . . . . . . . . . . . . . . . . . . . . . . . . 1412

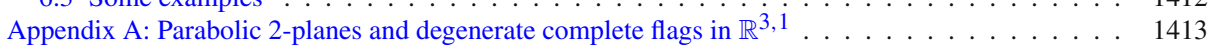

Appendix B: Heterotic supergravity in four Lorentzian dimensions . . . . . . . . . . . . . . . . . . 1414

References . . . . . . . . . . . . . . . . . . . . . . . . . . . . . 1417

\section{Introduction}

\subsection{Background and context}

Let $(M, g)$ be a pseudo-Riemannian manifold of signature $(p, q)$, equipped with a bundle of irreducible real Clifford modules $S$. If $(M, g)$ admits a spin structure, then $S$ carries a canonical connection $\nabla^{S}$ which lifts the Levi-Civita connection of $g$. This allows one to define the notions of parallel and Killing spinors, both of which were studied extensively in the literature $[8,16,58,77,88]$. Developments in supergravity and differential geometry (see 
references cited below) require the study of more general linear first-order partial differential equations for spinor fields. It is therefore convenient to develop a general framework which subsumes all such spinorial equations as special cases. In order to do this, we assume that $S$ is endowed with a fixed connection $\mathcal{D}: \Gamma(S) \rightarrow \Gamma\left(T^{*} M \otimes S\right.$ ) (which in practice will depend on various geometric structures on $(M, g)$ relevant to the specific problem under consideration) and consider the equation:

$$
\mathcal{D} \epsilon=0
$$

for a real spinor $\epsilon \in \Gamma(S)$. Solutions to this equation are called generalized Killing spinors with respect to $\mathcal{D}$ or simply $\mathcal{D}$-parallel spinors on $(M, g)$. We also consider linear constraints of the form:

$$
\mathcal{Q}(\epsilon)=0,
$$

where $Q \in \Gamma(\operatorname{Hom}(S, \mathcal{W} \otimes S))$, with $\mathcal{W}$ a vector bundle defined on $M$. Solutions $\epsilon \in \Gamma(M)$ of the system of Eqs. (1) and (2) are called constrained generalized Killing spinors on $(M, g)$.

The study of generalized Killing spinors can be motivated from various points of view, such as the theory of spinors on hypersurfaces $[9,23,29,33,78]$ or Riemannian geometry with torsion $[31,60]$. There is nowadays an extensive literature on the existence and properties of manifolds admitting generalized Killing spinors for specific connections $\mathcal{D}$ and in the presence of various spinorial structures, see for example $[2,3,32,55,57,59,76,79,80]$ and references therein.

Generalized Killing spinors play a fundamental role in supergravity and string theory $[45,84,85]$. They occur in these physics theories through the notion of "supersymmetric configuration", whose definition involves spinors parallel under a connection $\mathcal{D}$ on $S$ which is parameterized by geometric structures typically defined on fiber bundles, gerbes or Courant algebroids associated to $(M, g)[28,51,81]$. This produces the notion of supergravity Killing spinor equations - particular instances of (systems of) constrained generalized Killing spinor equations which are specific to the physics theory under consideration. Pseudo-Riemannian manifolds endowed with parameterizing geometric structures for which such equations admit non-trivial solutions are called supersymmetric configurations. They are called supersymmetric solutions if they also satisfy the equations of motion of the given supergravity theory. The study of supergravity Killing spinor equations was pioneered by Tod $[84,85]$ and later developed systematically in several references, including [7,10,11,19,27,40-43,63-66,70,71]. The study of supersymmetric solutions of supergravity theories provided an enormous boost to the subject of generalized Killing spinors and to spinorial geometry as a whole, which resulted in a large body of literature both in physics and mathematics, the latter of which is largely dedicated to the case of Euclidean signature in higher dimensional theories. We refer the reader to $[1,26,31,38,51,81]$ and references therein for more details and exhaustive lists of references.

Supergravity Killing spinor equations pose a number of new challenges when compared to simpler spinorial equations traditionally considered in the mathematics literature. First, supergravity Killing spinor equations must be studied for various theories and in various dimensions and signatures (usually Riemannian and Lorentzian), for real as well as complex spinors. In particular, this means that every single case in the modulo eight classification of real Clifford algebras must be considered, adding a layer of complexity to the problem. Second, such equations involve spinors parallel under non-canonical connections coupled to several other objects such as connections on gerbes, principal bundles or maps from the underlying manifold into a Riemannian manifold of special type. These objects, together with 
the underlying pseudo-Riemannian metric, must be treated as parameters of the supergravity Killing spinor equations, yielding a highly nontrivial non-linearly coupled system. Moreover, the formulation of supergravity theories relies on the Dirac-Penrose ${ }^{1}$ rather than on the Cartan approach to spinors. As a result, spinors appearing in such theories need not be associated to a spin structure or other a priory classical spinorial structure but involve the more general concept of a (real or complex) Lipschitz structure (see [35,67-69]). The latter naturally incorporates the 'R-symmetry' group of the theory and is especially well-adapted for geometric formulations of supergravity. Third, applications require the study of the moduli space of supersymmetric solutions of supergravity theories, involving the metric and all other geometric objects entering their formulation. This set-up yields remarkably nontrivial moduli problems for which the automorphism group(oid) of the system is substantially more complicated than the more familiar infinite-dimensional gauge group of automorphisms of a principal bundle or the diffeomorphism group of a compact manifold. Given these aspects, the study of supergravity Killing spinor equations and of moduli spaces of supersymmetric solutions of supergravity theories requires methods and techniques specifically dedicated to their understanding $[22,38,42,48,52-54,63,66-70]$. Developing such methods in a systematic manner is one of the goals of this article.

\subsection{Main results}

One approach to the study of supergravity Killing spinor equations is the so-called "method of bilinears" [42,84,85], which was successfully applied in various cases to simplify the local partial differential equations characterizing certain supersymmetric configurations and solutions. The idea behind this method is to consider the polyform constructed by taking the 'square' of the Killing spinor (instead of the spinor itself) and use the corresponding constrained generalized Killing spinor equations to extract a system of algebraic and partial differential equations for this polyform, thus producing necessary conditions for a constrained generalized Killing spinor to exist on $(M, g)$. These conditions can also be exploited to obtain information on the structure of supersymmetric solutions of the supergravity theory at hand. The main goal of the present work is to develop a framework inspired by these ideas aimed at investigating constrained generalized Killing spinors on pseudo-Riemannian manifolds by constructing a mathematical equivalence between real spinors and their polyform squares.

Whereas the fact that the 'square of a spinor' $[5,15,56]$ (see Definition 3.16 in Sect. 3) yields a polyform has been known for a long time (and the square of certain spinors with particularly nice stabilizers is well-known in specific—usually Riemannian—cases [15]), a proper mathematical theory to systematically characterize and compute spinor squares in every dimension and signature has been lacking so far. In this context, the fundamental questions to be addressed are $^{2}$ :

(1) What are the necessary and sufficient conditions for a polyform to be the square of a spinor, in every dimension and signature?

\footnotetext{
${ }^{1}$ When constructing such theories, one views spinors as sections of given bundles of Clifford modules. The existence of such bundles on the given space-time is postulated when writing down the theory, rather than deduced through the associated bundle construction from a specific classical spinorial structure assumed on to exist on that spacetime.

2 A systematic approach of this type was first used in references [64,65] for generalized Killing spinor equations in certain 8-dimensional flux compactifications of M-theory, using the Kähler-Atiyah bundle approach to such problems developed previously in $[63,66,70]$.
} 
(2) Can we (explicitly, if possible) translate constrained generalized Killing spinor equations into equivalent algebraic and partial differential equations for the square polyform?

In this work, we solve both questions for irreducible real spinors when the signature $(p, q)$ of the underlying pseudo-Riemannian manifold satisfies $p-q \equiv_{8} 0,2$, i.e. when the corresponding Clifford algebra is simple and of real type. We solve question (1) by fully characterizing the space of polyforms which are the (signed) square of spinors as the set of solutions of a system of algebraic equations which define a real affine variety in the space of polyforms. Every polyform solving this algebraic system can be written as the square of a real spinor which is determined up to a sign factor-and vice-versa. Following [63,66,70], the aforementioned algebraic system can be neatly written using the geometric product. The latter quantizes the wedge product, thereby deforming the exterior algebra to a unital associative algebra which is isomorphic to the Clifford algebra. This algebraic system can be considerably more complicated in indefinite signature than in the Euclidean case. On the other hand, we solve question (2) in the affirmative by reformulating constrained generalized Killing spinor equations on a spacetime $(M, g)$ of such signatures $(p, q)$ as an equivalent system of algebraic and partial differential equations for the square polyform. Altogether, this produces an equivalent reformulation of the constrained generalized Killing spinor problem as a more transparent and easier to handle system of partial differential equations for a polyform satisfying certain algebraic equations in the Kähler-Atiyah bundle of $(M, g)$. We believe that the framework developed in this paper is especially useful in pseudo-Riemannian signature and in higher dimensions, where the spin group does not act transitively on the unit sphere in spinor space and hence representation theory cannot be easily exploited to understand the square of a spinor in purely representation theoretic terms. One of our main results (see Theorem 4.26 for details and notation) is:

Theorem 1.1 Let $(M, g)$ be a connected, oriented and strongly spin pseudo-Riemannian manifold of signature $(p, q)$ and dimension $d=p+q$, such that $p-q \equiv_{8} 0,2$. Let $\mathcal{W}$ be a vector bundle on $M$ and $(S, \Gamma, \mathscr{B})$ be a paired real spinor bundle on $(M, g)$ whose admissible pairing has symmetry and adjoint types $\sigma, s \in\{-1,1\}$. Fix a connection $\mathcal{D}=\nabla^{S}-\mathcal{A}$ on $S$ (where $\mathcal{A} \in \Omega^{1}(M, E n d(S))$ ) and a morphism of vector bundles $\mathcal{Q} \in \Gamma(\operatorname{End}(S) \otimes \mathcal{W})$. Then there exists a nontrivial generalized Killing spinor $\epsilon \in \Gamma(S)$ with respect to the connection $\mathcal{D}$ which also satisfies the linear constraint $\mathcal{Q}(\epsilon)=0$ iff there exists a nowhere-vanishing polyform $\alpha \in \Omega(M)$ which satisfies the following algebraic and differential equations:

$$
\begin{gathered}
\alpha \diamond \beta \diamond \alpha=2^{\frac{d}{2}}(\alpha \diamond \beta)^{(0)} \alpha, \quad\left(\pi^{\frac{1-s}{2}} \circ \tau\right)(\alpha)=\sigma \alpha, \\
\nabla^{g} \alpha=\hat{\mathcal{A}} \diamond \alpha+\alpha \diamond\left(\pi^{\frac{1-s}{2}} \circ \tau\right)(\hat{\mathcal{A}}), \quad \hat{\mathcal{Q}} \diamond \alpha=0
\end{gathered}
$$

for every polyform $\beta \in \Omega(M)$, where $\hat{\mathcal{A}} \in \Omega^{1}\left(M, \wedge T^{*} M\right)$ and $\hat{\mathcal{Q}} \in \Gamma\left(\wedge T^{*} M \otimes \mathcal{W}\right)$ are the symbols of $\mathcal{A}$ and $\mathcal{Q}$ while $\pi, \tau$ are the canonical automorphism and anti-automorphism of the Kähler-Atiyah bundle $\left(\wedge T^{*} M, \diamond\right)$ of $(M, g)$. If $\epsilon$ is chiral of chirality $\mu \in\{-1,1\}$, then we have to add the condition:

$$
*(\pi \circ \tau)(\alpha)=\mu \alpha,
$$

where $*$ is the Hodge operator of $(M, g)$. Moreover, any such polyform $\alpha$ determines a nowhere-vanishing real spinor $\epsilon \in \Gamma(S)$, which is unique up to a sign and satisfies the constrained generalized Killing spinor equations with respect to $\mathcal{D}$ and $\mathcal{Q}$. 
When $(M, g)$ is a Lorenzian four-manifold, we say that a pair of nowhere-vanishing oneforms $(u, l)$ defined on $M$ is parabolic if $u$ and $l$ are mutually orthogonal with $u$ null and $l$ spacelike of unit norm. Applying the previous result, we obtain (see Theorem 4.32 and Sect. 4.6 for detail and terminology):

Theorem 1.2 Let $(M, g)$ be a connected and spin Lorentzian four-manifold of "mostly plus" signature such that $H^{1}\left(M, \mathbb{Z}_{2}\right)=0$ and $S$ be a real spinor bundle associated to the spin structure of $(M, g)$ (which is unique up to isomorphism). Then there exists a natural bijection between the set of global smooth sections of the projective bundle $\mathbb{P}(S)$ and the set of trivializable and co-oriented distributions $(\Pi, \mathcal{H})$ of parabolic 2-planes in $T^{*} M$. Moreover, there exist natural bijections between the following two sets:

(a) The set $\Gamma(\dot{S}) / \mathbb{Z}_{2}$ of sign-equivalence classes of nowhere-vanishing real spinors $\epsilon \in$ $\Gamma(S)$.

(b) The set of strong equivalence classes of parabolic pairs of one-forms $(u, l) \in \mathcal{P}(M, g)$.

In particular, the sign-equivalence class of a nowhere-vanishing spinor $\epsilon \in \Gamma(S)$ determines and is determined by a parabolic pair of one-forms $(u, l)$ considered up to transformations of the form $(u, l) \rightarrow(-u, l)$ and $l \rightarrow l+c u$ with $c \in \mathbb{R}$.

We use this result to characterize spin Lorentzian four-manifolds $(M, g)$ with $H^{1}(M, g)=$ 0 which admit real Killing spinors and supersymmetric bosonic heterotic configurations associated to "paired principal bundles" $(P, \mathfrak{c})$ over such a manifold through systems of partial differential equations for $u$ and $l$, which we explore in specific cases. Taking $(M, g)$ to be of signature $(3,1)$, we prove the following results (see Theorems 5.3 and 6.6), where $*$ and $\mathrm{d}^{*}$ denote the Hodge operator and codifferential of $(M, g)$ while $\nabla^{g}$ denotes the action of its Levi-Civita on covariant tensors:

Theorem $1.3(M, g)$ admits a nontrivial real Killing spinor with Killing constant $\frac{\lambda}{2} \in \mathbb{R}$ iff it admits a parabolic pair of one-forms $(u, l)$ which satisfies:

$$
\nabla^{g} u=\lambda u \wedge l, \quad \nabla^{g} l=\lambda(l \otimes l-g)+\kappa \otimes u
$$

for some $\kappa \in \Omega^{1}(M)$. In this case, $u^{\sharp} \in \mathfrak{X}(M)$ is a Killing vector field with geodesic integral curves.

Theorem 1.4 A bosonic heterotic configuration $(g, \varphi, H, A)$ of $(M, P, \mathfrak{c})$ is supersymmetric iff there exists a parabolic pair of one-forms $(u, l)$ which satisfies (here $\rho \stackrel{\text { def. }}{=} * H \in \Omega^{1}(M)$ ):

$$
\begin{aligned}
& \varphi \wedge u=*(\rho \wedge u), \varphi \wedge u \wedge l=-g^{*}(\rho, l) * u, \quad-g^{*}(\varphi, l) u=*(l \wedge u \wedge \rho), \\
& g^{*}(u, \varphi)=0, \quad g^{*}(u, \rho)=0, \quad g^{*}(\rho, \varphi)=0, \quad F_{A}=u \wedge \chi_{A}, \\
& \nabla^{g} u=\frac{1}{2} u \wedge \varphi, \quad \nabla^{g} l=\frac{1}{2} *(\rho \wedge l)+\kappa \otimes u, \quad d^{*} \rho=0
\end{aligned}
$$

for some one-form $\kappa \in \Omega^{1}(M)$ and some $\mathfrak{g}_{P}$-valued one-form $\chi_{A} \in \Omega^{1}\left(M, \mathfrak{g}_{P}\right)$ which is orthogonal to $u$. In this case, $u^{\sharp} \in \mathfrak{X}(M)$ is a Killing vector field.

Let $\mathbb{H}$ be the Poincaré half plane with coordinates $x \in \mathbb{R}, y \in \mathbb{R}_{>0}$. Using the last result above, we show (see Sect. 5.4) that the following one-parameter family of metrics defined on $\mathbb{R}^{2} \times \mathbb{H}$ :

$$
\mathrm{d} s_{g}^{2}=\mathcal{F}\left(\mathrm{d} x^{v}\right)^{2}+\frac{\mathrm{d} x^{v} \mathrm{~d} x^{u}}{y^{2}}+\frac{(\mathrm{d} x)^{2}+(\mathrm{d} y)^{2}}{\lambda^{2} y^{2}} \quad(\lambda \in \mathbb{R})
$$


(where $\mathbb{R}^{2}$ has Cartesian coordinates $x^{v}, x^{u}$ ) admits real Killing spinors for every $\mathcal{F} \in$ $C^{\infty}(\mathbb{H})$. We also show that these metrics are Einstein with Einstein constant $\Lambda=-3 \lambda^{2}$ when $\mathcal{F}$ has the form:

$$
\mathcal{F}=\left(a_{1}+a_{2} x\right)\left(a_{3} y+\frac{a_{4}}{y^{2}}\right) \text { or } \mathcal{F}=\left(a_{1} e^{c x}+a_{2} e^{-c x}\right)\left[a_{3} \mathrm{BY}(c y)+a_{4} \mathrm{BJ}(c y)\right],
$$

where BY and BJ are the spherical Bessel functions and $a_{1}, \ldots, a_{4} \in \mathbb{R}$. These give deformations of the $\mathrm{AdS}_{4}$ spacetime of cosmological constant $\Lambda$, which obtains for $a_{1}=a_{2}=$ $a_{3}=a_{4}=0$.

\subsection{Open problems and further directions}

Theorem 1.1 refers exclusively to real spinors in signature $p-q \equiv_{8} 0,2$, for which the irreducible Clifford representation map is an isomorphism. It would be interesting to extend this result to the remaining signatures, which encompass two main cases, namely real spinors of complex and quaternionic type (the latter of which can be reducible). This would yield a rich reformulation of the theory of real spinors through polyforms subject to algebraic constraints, which could be used to study generalized Killing spinors in all dimensions and signatures. It would also be interesting to extend Theorem 1.1 to other types of spinorial equations, such as those characterizing harmonic or twistor spinors and generalizations thereof, investigating if it is possible to develop an equivalent theory exclusively in terms of polyforms.

Some important signatures satisfy the condition $p-q \equiv_{8} 0,2$, most notably signatures $(2,0),(1,1),(3,1)$ and $(9,1)$. The latter two are especially relevant to supergravity theories and one could apply the formalism developed in this article to study moduli spaces of supersymmetric solutions in these cases. Several open problems of analytic, geometric and topological type exist regarding the heterotic system in four and ten Lorentzian dimensions, as the mathematical study of its Riemannian analogue shows [38]. Most problems related to existence, classification, construction of examples and moduli are open and give rise to interesting analytic and geometric questions on Lorentzian four-manifolds and ten-manifolds. In this direction, we hope that Appendix B can serve as a brief introduction to heterotic supergravity in four Lorentzian dimensions for mathematicians who may be interested in such questions. The local structure of ten-dimensional supersymmetric solutions to heterotic supergravity was explored in $[49,50,82]$, where that problem was reduced to a minimal set of partial differential equations on a local Lorentzian manifold of special type.

\subsection{Outline of the paper}

Section 2 gives the description of rank-one endomorphisms of a vector space which are (anti)symmetric with respect to a non-degenerate bilinear pairing assumed to be symmetric or skew-symmetric. Section 3 develops the algebraic theory of the square of a spinor culminating in Theorem 3.20, which characterizes it through a system of algebraic conditions in the Kähler-Atiyah algebra of the underlying quadratic vector space. In Sect. 4, we apply this to real spinors on pseudo-Riemannian manifolds of signature $(p, q)$ satisfying $p-q \equiv 8$ 0,2 , obtaining a complete characterization of generalized constrained Killing spinors as polyforms satisfying algebraic and partial differential equations which we list explicitly. Section 5 applies this theory to real Killing spinors in four Lorentzian dimensions, obtaining a new global characterization of such. In Sect. 6, we apply the same theory to the study of 
supersymmetric configurations of heterotic supergravity, whose mathematical formulation is explained briefly in an appendix.

Notations and conventions Throughout the paper, we use Einstein summation over repeated indices. We let $R^{\times}$denote the group of invertible elements of any commutative ring $R$. In particular, the multiplicative group of non-zero real numbers is denoted by $\mathbb{R}^{\times}=\mathbb{R}$. For any positive integer $n$, the symbol $\equiv_{n}$ denotes the equivalence relation of congruence of integers modulo $n$, while $\mathbb{Z}_{n}=\mathbb{Z} / n \mathbb{Z}$ denotes the corresponding congruence group. All manifolds considered in the paper are assumed to be smooth, connected and paracompact, while all fiber bundles are smooth. The set of globally-defined smooth sections of any fiber bundle $F$ defined on a manifold $M$ is denoted by $\Gamma(F)$. We denote by $G_{0}$ the connected component of the identity of any Lie group $G$. Given a vector bundle $S$ on a manifold $M$, the dual vector bundle is denoted by $S^{*}$ while the bundle of endomorphisms is denoted by $\operatorname{End}(S) \simeq S^{*} \otimes S$. The trivial real line bundle on $M$ is denoted by $\mathbb{R}_{M}$. The space of globally-defined smooth sections of $S$ is denoted by $\Gamma(S)$, while the set of those globally-defined smooth sections of $S$ which do not vanish anywhere on $M$ is denoted by $\Gamma(S)$. The complement of the origin in any $\mathbb{R}$-vector space $\Sigma$ is denoted by $\dot{\Sigma}$ while the complement of the image $0_{S}$ of the zero section of a vector bundle $S$ defined on a manifold $M$ is denoted by $\dot{S}$. The inclusion $\dot{\Gamma}(S) \subset \Gamma(S)$ is generally strict. If $A$ is any subset of the total space of $S$, we define:

$$
\Gamma(A) \stackrel{\text { def. }}{=}\left\{s \in \Gamma(S) \mid s_{m} \in A \cap S_{m} \quad \forall m \in M\right\} \subset \Gamma(S) .
$$

Notice the relation $\Gamma(S)=\Gamma(\dot{S})$. All pseudo-Riemannian manifolds $(M, g)$ are assumed to have dimension at least two and signature $(p, q)$ satisfying $p-q \equiv_{8} 0,2$; in particular, all Lorentzian four-manifolds have "mostly plus" signature $(3,1)$. For any pseudo-Riemannian manifold $(M, g)$, we denote by $\langle,\rangle_{g}$ the (generally indefinite) metric induced by $g$ on the

total exterior bundle $\Lambda(M) \stackrel{\text { def. }}{=} \wedge T^{*} M=\oplus_{k=0}^{\operatorname{dim} M} \wedge^{k} T^{*} M$. We denote by $\nabla^{g}$ the Levi-Civita connection of $g$ and use the same symbol for its action on tensors. The equivalence class of an element $\xi$ of an $\mathbb{R}$-vector space $\Sigma$ under the sign action of $\mathbb{Z}_{2}$ on $\Sigma$ is denoted by $\hat{\xi} \in \Sigma / \mathbb{Z}_{2}$ and called the sign equivalence class of $\xi$.

\section{Representing real vectors as endomorphisms in a paired vector space}

Let $\Sigma$ be an $\mathbb{R}$-vector space of positive even dimension $N \geq 2$, equipped with a nondegenerate bilinear pairing $\mathscr{B}: \Sigma \times \Sigma \rightarrow \mathbb{R}$, which we assume to be either symmetric or skew-symmetric. In this situation, the pair $(\Sigma, \mathscr{B})$ is called a paired vector space. We say that $\mathscr{B}($ or $(\Sigma, \mathscr{B}))$ has symmetry type $\sigma \in\{-1,1\}$ if:

$$
\mathscr{B}\left(\xi_{1}, \xi_{2}\right)=\sigma \mathscr{B}\left(\xi_{2}, \xi_{1}\right) \quad \forall \xi_{1}, \xi_{2} \in \Sigma \text {. }
$$

Thus $\mathscr{B}$ is symmetric if it has symmetry type +1 and skew-symmetric if it has symmetry type -1 . Let $(\operatorname{End}(\Sigma), 0)$ be the unital associative $\mathbb{R}$-algebra of linear endomorphisms of $\Sigma$, where $\circ$ denotes composition of linear maps. Given $E \in \operatorname{End}(\Sigma)$, let $E^{t} \in \operatorname{End}(\Sigma)$ denote the adjoint of $E$ taken with respect to $\mathscr{B}$, which is uniquely determined by the condition:

$$
\mathscr{B}\left(\xi_{1}, E\left(\xi_{2}\right)\right)=\mathscr{B}\left(E^{t}\left(\xi_{1}\right), \xi_{2}\right) \quad \forall \xi_{1}, \xi_{2} \in \Sigma
$$

The map $E \rightarrow E^{t}$ is a unital anti-automorphism of the $\mathbb{R}$-algebra $(\operatorname{End}(\Sigma), \circ)$. 


\subsection{Tame endomorphisms and the squaring maps}

Definition 2.1 An endomorphism $E \in \operatorname{End}(\Sigma)$ is called tame if its rank satisfies $\operatorname{rk}(E) \leq 1$.

Thus $E$ is tame iff it vanishes or is of unit rank. Let:

$$
\mathcal{T}:=\mathcal{T}(\Sigma) \stackrel{\text { def. }}{=}\{E \in \operatorname{End}(\Sigma) \mid \operatorname{rk}(E) \leq 1\} \subset \operatorname{End}(\Sigma)
$$

be the real determinantal variety of tame endomorphisms of $\Sigma$ and:

$$
\dot{\mathcal{T}}:=\dot{\mathcal{T}}(\Sigma) \stackrel{\text { def. }}{=} \mathcal{T} \backslash\{0\}=\{E \in \operatorname{End}(\Sigma) \mid \operatorname{rk}(E)=1\}
$$

be its open subset consisting of endomorphisms of rank one. We view $\mathcal{T}$ as a real affine variety of dimension $2 N-1$ in the vector space $\operatorname{End}(\Sigma) \simeq \mathbb{R}^{N^{2}}$ and $\dot{\mathcal{T}}$ as a semi-algebraic variety. Elements of $\mathcal{T}$ can be written as:

$$
E=\xi \otimes \beta,
$$

for some $\xi \in \Sigma$ and $\beta \in \Sigma^{*}$, where $\Sigma^{*}=\operatorname{Hom}(\Sigma, \mathbb{R})$ denotes the vector space dual to $\Sigma$. Notice that $\operatorname{tr}(E)=\beta(\xi)$. When $E \in \mathcal{T}$ is non-zero, the vector $\xi$ and the linear functional $\beta$ appearing in the relation above are non-zero and determined by $E$ up to transformations of the form $(\xi, \beta) \rightarrow\left(\lambda \xi, \lambda^{-1} \beta\right)$ with $\lambda \in \mathbb{R}^{\times}$. In particular, $\dot{\mathcal{T}}$ is a manifold diffeomorphic with the quotient $\left(\mathbb{R}^{N} \backslash\{0\}\right) \times\left(\mathbb{R}^{N} \backslash\{0\}\right) / \mathbb{R}^{\times}$, where $\mathbb{R}^{\times}$acts with weights +1 and -1 on the two copies of $\mathbb{R}^{N} \backslash\{0\}$.

Definition 2.2 The signed squaring maps of a paired vector space $(\Sigma, \mathscr{B})$ are the quadratic maps $\mathcal{E}_{ \pm}: \Sigma \rightarrow \mathcal{T}$ defined through:

$$
\mathcal{E}_{ \pm}(\xi)= \pm \xi \otimes \xi^{*} \quad \forall \xi \in \Sigma,
$$

where $\xi^{*} \stackrel{\text { def. }}{=} \mathscr{B}(-, \xi) \in \Sigma^{*}$ is the linear map dual to $\xi$ relative to $\mathscr{B}$. The map $\mathcal{E}_{+}$is called the positive squaring map of $(\Sigma, \mathscr{B})$, while $\mathcal{E}_{-}$is called the negative squaring map of $(\Sigma, \mathscr{B})$.

Let $\kappa \in\{-1,1\}$ be a sign factor and consider the open semi-algebraic set $\dot{\Sigma} \stackrel{\text { def. }}{=} \Sigma \backslash\{0\}$. Notice that $\mathcal{E}_{ \pm}(\xi)=0$ iff $\xi=0$, hence $\mathcal{E}_{ \pm}(\dot{\Sigma}) \subset \dot{\mathcal{T}}$. Let $\dot{\mathcal{E}}_{ \pm}: \dot{\Sigma} \rightarrow \dot{\mathcal{T}}$ be the restrictions of $\mathcal{E}_{ \pm}$to $\dot{\Sigma}$. The proof of the following lemma is immediate.

Lemma 2.3 For each $\kappa \in\{-1,1\}$, the restricted quadratic map $\dot{\mathcal{E}}_{\kappa}: \dot{\Sigma} \rightarrow \dot{\mathcal{T}}$ is two-to-one, namely:

$$
\dot{\mathcal{E}}_{\kappa}^{-1}\left(\left\{\kappa \xi \otimes \xi^{*}\right\}\right)=\{-\xi, \xi\} \quad \forall \xi \in \dot{\Sigma} .
$$

Moreover, we have $\mathcal{E}_{\kappa}(\xi)=0$ iff $\xi=0$ and hence $\mathcal{E}_{\kappa}$ is a real branched double cover of its image, which is ramified at the origin.

\subsection{Admissible endomorphisms}

The maps $\mathcal{E}_{ \pm}$need not be surjective. To characterize their images, we introduce the notion of admissible endomorphism. Let $(\Sigma, \mathscr{B})$ be a paired vector space of type $\sigma$.

Definition 2.4 An endomorphism $E$ of $\Sigma$ is called $\mathscr{B}$-admissible if it satisfies the conditions:

$$
E \circ E=\operatorname{tr}(E) E \text { and } E^{t}=\sigma E \text {. }
$$


Let:

$$
\mathcal{C}:=\mathcal{C}(\Sigma, \mathscr{B}) \stackrel{\text { def. }}{=}\left\{E \in \operatorname{End}(\Sigma) \mid E \circ E=\operatorname{tr}(E) E, E^{t}=\sigma E\right\}
$$

denote the real cone of $\mathscr{B}$-admissible endomorphisms of $\Sigma$.

Remark 2.5 Tame endomorphisms are not related to admissible endomorphisms in any simple way. A tame endomorphism need not be admissible, since it need not be (anti-)symmetric with respect to $\mathscr{B}$. An admissible endomorphism need not be tame, since it can have rank larger than one (as shown by a quick inspection of explicit examples in four dimensions).

Let:

$$
\mathcal{Z}:=\mathcal{Z}(\Sigma, \mathscr{B}) \stackrel{\text { def. }}{=} \mathcal{T}(\Sigma) \cap \mathcal{C}(\Sigma, \mathscr{B})
$$

denote the real cone of those endomorphisms of $E$ which are both tame and $\mathscr{B}$-admissible and consider the open set $\dot{\mathcal{Z}} \stackrel{\text { def. }}{=} \mathcal{Z} \backslash\{0\}$.

Lemma 2.6 We have:

$$
\mathcal{Z}=\operatorname{Im}\left(\mathcal{E}_{+}\right) \cup \operatorname{Im}\left(\mathcal{E}_{-}\right) \text {and } \operatorname{Im}\left(\mathcal{E}_{+}\right) \cap \operatorname{Im}\left(\mathcal{E}_{-}\right)=\{0\} .
$$

Hence an endomorphism $E \in \operatorname{End}(\Sigma)$ belongs to $\dot{\mathcal{Z}}$ iff there exists a non-zero vector $\xi \in \dot{\Sigma}$ and a sign factor $\kappa \in\{-1,1\}$ such that:

$$
E=\mathcal{E}_{\kappa}(\xi) .
$$

Moreover, $\kappa$ is uniquely determined by $E$ through this equation while $\xi$ is determined up to sign.

Proof Let $E \in \dot{\mathcal{Z}}$. Since $E$ has unit rank, there exists a non-zero vector $\xi \in \Sigma$ and a non-zero linear functional $\beta \in \Sigma^{*}$ such that $E=\xi \otimes \beta$. Since $\mathscr{B}$ is non-degenerate, there exists a unique non-zero $\xi_{0} \in \Sigma$ such that $\beta=\mathscr{B}\left(-, \xi_{0}\right)=\xi_{0}^{*}$. The condition $E^{t}=\sigma E$ amounts to:

$$
\mathscr{B}\left(-, \xi_{0}\right) \xi=\mathscr{B}(-, \xi) \xi_{0} .
$$

Since $\mathscr{B}$ is non-degenerate, there exists $\chi \in \Sigma$ such that $\mathscr{B}(\chi, \xi) \neq 0$, which by the previous equations also satisfies $\mathscr{B}\left(\chi, \xi_{0}\right) \neq 0$. Hence:

$$
\xi_{0}=\frac{\mathscr{B}\left(\chi, \xi_{0}\right)}{\mathscr{B}(\chi, \xi)} \xi=\frac{\mathscr{B}\left(\xi_{0}, \chi\right)}{\mathscr{B}(\xi, \chi)} \xi
$$

and:

$$
E=\frac{\mathscr{B}\left(\xi_{0}, \chi\right)}{\mathscr{B}(\xi, \chi)} \xi \otimes \xi^{*}
$$

Using the rescaling $\xi \mapsto \xi^{\prime} \stackrel{\text { def. }}{=}\left|\frac{\mathscr{B}(\xi 0, \chi)}{\mathscr{B}(\xi, \chi)}\right|^{\frac{1}{2}} \xi$, the previous relation gives $E=\kappa \xi^{\prime} \otimes\left(\xi^{\prime}\right)^{*} \in$ $\operatorname{Im}\left(\mathcal{E}_{\kappa}\right)$, where $\kappa \stackrel{\text { def. }}{=} \operatorname{sign}\left(\frac{\mathscr{B}\left(\xi_{0}, \chi\right)}{\mathscr{B}(\xi, \chi)}\right)$. This implies the inclusion $\mathcal{Z} \subseteq \operatorname{Im}\left(\mathcal{E}_{+}\right) \cup \operatorname{Im}\left(\mathcal{E}_{-}\right)$. Lemma 2.3 now shows that $\xi^{\prime}$ is unique up to sign. The inclusion $\operatorname{Im}\left(\mathcal{E}_{+}\right) \cup \operatorname{Im}\left(\mathcal{E}_{-}\right) \subseteq \mathcal{Z}$ follows by direct computation using the explicit form $E=\kappa \xi \otimes \xi^{*}$ of an endomorphism $E \in \operatorname{Im}\left(\mathcal{E}_{\kappa}\right)$, which implies:

$$
E \circ E=\mathscr{B}(\xi, \xi) E, \quad E^{t}=\sigma E, \operatorname{tr}(E)=\mathscr{B}(\xi, \xi) .
$$


Combining the two inclusions above gives $\mathcal{Z}=\operatorname{Im}\left(\mathcal{E}_{+}\right) \cup \operatorname{Im}\left(\mathcal{E}_{-}\right)$. The relation $\operatorname{Im}\left(\mathcal{E}_{+}\right) \cap$ $\operatorname{Im}\left(\mathcal{E}_{-}\right)=\{0\}$ follows immediately from Lemma 2.3 .

Definition 2.7 The signature $\kappa_{E} \in\{-1,1\}$ of an element $E \in \dot{\mathcal{Z}}$ with respect to $\mathscr{B}$ is the sign factor $\kappa$ determined by $E$ as in Lemma 2.6. When $E=0$, we set $\kappa_{E}=0$.

Remark 2.8 Notice that $\kappa_{-E}=-\kappa_{E}$ for all $E \in \mathcal{Z}$.

In view of the above, define:

$$
\mathcal{Z}_{ \pm}:=\mathcal{Z}_{ \pm}(\Sigma, \mathscr{B}) \stackrel{\text { def. }}{=} \operatorname{Im}\left(\mathcal{E}_{ \pm}\right) .
$$

Then:

$$
\mathcal{Z}_{-}=-\mathcal{Z}_{+}, \quad \mathcal{Z}=\mathcal{Z}_{+} \cup \mathcal{Z}_{-} \text {and } \mathcal{Z}_{+} \cap \mathcal{Z}_{-}=\{0\}
$$

Let $\mathbb{Z}_{2} \simeq\{-1,1\}$ act on $\Sigma$ and on $\mathcal{Z} \subset \operatorname{End}(E)$ by sign multiplication. Then $\mathcal{E}_{+}$and $\mathcal{E}_{-}$ induce the same map between the quotients (which is a bijection by Lemma 2.6). We denote this map by:

$$
\hat{\mathcal{E}}: \Sigma / \mathbb{Z}_{2} \stackrel{\sim}{\rightarrow} \mathcal{Z} / \mathbb{Z}_{2}
$$

Definition 2.9 The bijection (8) is called the class squaring map of $(\Sigma, \mathscr{B})$.

\subsection{The manifold $\dot{\mathcal{Z}}$ and the projective squaring map}

Given any endomorphism $A \in \operatorname{End}(\Sigma)$, define a (possibly degenerate) bilinear pairing $\mathscr{B}_{A}$ on $\Sigma$ through:

$$
\mathscr{B}_{A}\left(\xi_{1}, \xi_{2}\right) \stackrel{\text { def. }}{=} \mathscr{B}\left(\xi_{1}, A\left(\xi_{2}\right)\right) \quad \forall \xi_{1}, \xi_{2} \in \Sigma .
$$

Notice that $\mathscr{B}_{A}$ is symmetric iff $A^{t}=\sigma A$ and skew-symmetric iff $A^{t}=-\sigma A$.

Proposition 2.10 The open set $\dot{\mathcal{Z}}$ has two connected components, which are given by:

$$
\dot{\mathcal{Z}}_{+} \stackrel{\text { def. }}{=} \operatorname{Im}\left(\dot{\mathcal{E}}_{+}\right)=\operatorname{Im}\left(\mathcal{E}_{+}\right) \backslash\{0\} \text { and } \dot{\mathcal{Z}}_{-} \stackrel{\text { def. }}{=} \operatorname{Im}\left(\dot{\mathcal{E}}_{-}\right)=\operatorname{Im}\left(\mathcal{E}_{-}\right) \backslash\{0\},
$$

and satisfy:

$$
\begin{aligned}
& \dot{\mathcal{Z}}_{+}=\left\{E \in \mathcal{Z} \mid \kappa_{E}=+1\right\} \subset\left\{E \in \mathcal{Z} \mid \mathscr{B}_{E} \geq 0\right\}, \\
& \dot{\mathcal{Z}}_{-}=\left\{E \in \mathcal{Z} \mid \kappa_{E}=-1\right\} \subset\left\{E \in \mathcal{Z} \mid \mathscr{B}_{E} \leq 0\right\} .
\end{aligned}
$$

Moreover, the maps $\dot{\mathcal{E}}_{ \pm}: \dot{\Sigma} \rightarrow \dot{\mathcal{Z}}_{ \pm}$define principal $\mathbb{Z}_{2}$-bundles over $\dot{\mathcal{Z}}_{ \pm}$.

Proof By Lemma 2.6, we know that $\dot{\mathcal{Z}}=\dot{\mathcal{Z}}_{+} \cup \dot{\mathcal{Z}}_{-}$and $\dot{\mathcal{Z}}_{+} \cap \dot{\mathcal{Z}}_{-}=\emptyset$. The open set $\dot{\Sigma}$ is connected because $N=\operatorname{dim} \Sigma \geq 2$. Fix $\kappa \in\{-1,1\}$. Since the continuous map $\mathcal{E}_{\kappa}$ surjects onto $\dot{\mathcal{Z}}_{\kappa}$, it follows that $\dot{\mathcal{Z}}_{\kappa}$ is connected. Let $E \in \dot{\mathcal{Z}}_{\kappa}$. The pairing $\mathscr{B} E$ is symmetric since $E^{t}=\sigma E$. By Lemma 2.6, we have $E=\kappa \mathscr{B}\left(-, \xi_{0}\right) \xi_{0}$ for some non-zero $\xi_{0} \in \Sigma$ and hence:

$$
\mathscr{B} E(\xi, \xi)=\mathscr{B}(\xi, E(\xi))=\kappa\left|\mathscr{B}\left(\xi, \xi_{0}\right)\right|^{2} \quad \forall \xi \in \Sigma .
$$

Since $\xi_{0} \neq 0$ and $\mathscr{B}$ is non-degenerate, this shows that $\mathscr{B}_{E}$ is nontrivial and that it is positive-semidefinite when restricted to $\dot{\mathcal{Z}}_{+}$and negative-semidefinite when restricted to

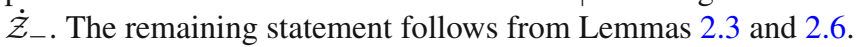


Proposition $2.11 \dot{\mathcal{Z}}(\Sigma, \mathscr{B})$ is a manifold diffeomorphic to $\mathbb{R}^{\times} \times \mathbb{R P}^{N-1}$ (where $N=\operatorname{dim} \Sigma$ ).

Proof Let $|\cdot|_{0}^{2}$ denotes the norm induced by any scalar product on $\Sigma$. Then the diffeomorphism:

$$
\dot{\mathcal{Z}} \stackrel{\sim}{\rightarrow} \mathbb{R}^{\times} \times \mathbb{R} \mathbb{P}^{N-1}, \quad \kappa \xi \otimes \xi^{*} \mapsto\left(\kappa|\xi|_{0}^{2},[\xi]\right)
$$

satisfies the desired properties.

The maps $\dot{\mathcal{E}}_{ \pm}: \dot{\Sigma} \rightarrow \operatorname{End}(\Sigma) \backslash\{0\}$ induce the same map:

$$
\mathbb{P} \mathcal{E}: \mathbb{P}(\Sigma) \rightarrow \mathbb{P}(\operatorname{End}(\Sigma)), \quad[\xi] \mapsto\left[\xi \otimes \xi^{*}\right],
$$

between the projectivizations $\mathbb{P}(\Sigma)$ and $\mathbb{P}(\operatorname{End}(\Sigma))$ of the real vector spaces $\Sigma$ and $\operatorname{End}(\Sigma)$. Setting $\mathbb{P} \mathcal{Z}(\Sigma, \mathscr{B}) \stackrel{\text { def. }}{=} \dot{\mathcal{Z}}(\Sigma, \mathscr{B}) / \mathbb{R}^{\times} \subset \mathbb{P E n d}(\Sigma)$, Proposition 2.11 gives:

Proposition 2.12 The map $\mathbb{P} \mathcal{E}: \mathbb{P}(\Sigma) \rightarrow \mathbb{P} \mathcal{Z}(\Sigma, \mathscr{B})$ is a diffeomorphism.

Definition 2.13 $\mathbb{P} \mathcal{E}: \mathbb{P}(\Sigma) \stackrel{\sim}{\rightarrow} \mathbb{P} \mathcal{Z}(\Sigma, \mathscr{B})$ is called the projective squaring map of $(\Sigma, \mathscr{B})$.

\subsection{Tamings of $\mathscr{B}$}

The bilinear form $\mathscr{B}_{A}$ defined in Eq. (9) is non-degenerate iff the endomorphism $A \in \operatorname{End}(\Sigma)$ is invertible. The following result is immediate:

Proposition 2.14 Let $\mathscr{B}^{\prime}$ be a non-degenerate symmetric pairing on $\Sigma$. Then there exists a unique endomorphism $A \in G L(E)$ (called the operator of $\mathscr{B}^{\prime}$ with respect to $\mathscr{B}$ ) such that $\mathscr{B}^{\prime}=\mathscr{B}_{A}$. Moreover, $A$ is invertible and satisfies $A^{t}=\sigma A$. Furthermore, the transpose $E^{T}$ of any endomorphism $E \in \operatorname{End}(\Sigma)$ with respect to $\mathscr{B}^{\prime}$ is given by:

$$
E^{T}=\left(A^{-1}\right)^{t} E^{t} A^{t}=A^{-1} E^{t} A
$$

and in particular we have $A^{T}=A^{t}=\sigma A$.

Remark 2.15 Replacing $\xi_{2}$ by $A^{-1} \xi_{2}$ in the relation $\mathscr{B}^{\prime}\left(\xi_{1}, \xi_{2}\right)=\mathscr{B}\left(\xi_{1}, A \xi_{2}\right)$ gives:

$$
\mathscr{B}\left(\xi_{1}, \xi_{2}\right)=\mathscr{B}^{\prime}\left(\xi_{1}, A^{-1} \xi_{2}\right),
$$

showing that $A^{-1}$ is the operator of $\mathscr{B}$ with respect to $\mathscr{B}^{\prime}$.

Definition 2.16 We say that $A \in \operatorname{End}(\Sigma)$ is a taming of $\mathscr{B}$ if $\mathscr{B}_{A}$ is a scalar product.

Let $A \in \operatorname{End}(\Sigma)$ be a taming of $\mathscr{B}$ and denote by $(-,-) \stackrel{\text { def. }}{=} \mathscr{B}_{A}$ the corresponding scalar product. Relation (11) shows that the matrix $\hat{B}$ of $\mathscr{B}$ with respect to a (, )-orthonormal basis $\left\{e_{1}, \ldots, e_{N}\right\}$ of $\Sigma$ is the inverse of the matrix $\hat{A}$ of $A$ in the the same basis. Distinguish the cases:

1. When $\mathscr{B}$ is symmetric, its operator $B$ with respect to (, ) and the taming $A=B^{-1}$ of $\mathscr{B}$ are (, )-symmetric and can be diagonalized by a $(-,-)$-orthogonal linear transformation of $\Sigma$. If $(p, q)$ is the signature of $\mathscr{B}$, Sylvester's theorem shows that we can choose the basis $\left\{e_{i}\right\}$ such that:

$$
\hat{A}=\operatorname{diag}(+1, \ldots,+1,-1, \ldots,-1),
$$

with $p$ positive and $q$ negative entries. With this choice, we have $A^{2}=$ Id. 
2. When $\mathscr{B}$ is skew-symmetric, we have:

$$
\mathscr{B}\left(\xi_{1}, \xi_{2}\right)=-\left(\xi_{1}, J\left(\xi_{2}\right)\right) \text {, i.e. }\left(\xi_{1}, \xi_{2}\right)=\mathscr{B}\left(\xi_{1}, J\left(\xi_{2}\right)\right),
$$

where $J$ is a $(-,-)$-compatible complex structure on $\Sigma$. This gives $A^{-1}=-J$ and hence $A=J$, which is antisymmetric with respect to both $(-,-)$ and $\mathscr{B}$. Setting $N=2 n$, we can choose $\left\{e_{1}, \ldots, e_{n}\right\}$ to be a basis of $\Sigma$ over $\mathbb{C}$ which is orthonormal with respect to the Hermitian scalar product defined by $(-,-)$ and $J$ and take $e_{n+i}=J e_{i}$ for all $i=1, \ldots, n$. Then the basis $\left\{e_{1}, \ldots, e_{N}\right\}$ over $\mathbb{R}$ is $(-,-)$-orthonormal while being a Darboux basis for $\mathscr{B}$ and we have:

$$
\hat{A}=\hat{J}=\left[\begin{array}{cc}
0 & I_{n} \\
-I_{n} & 0
\end{array}\right]
$$

where $I_{n}$ is the identity matrix of size $n$.

Proposition 2.17 Let $\mathscr{B}^{\prime}$ be a non-degenerate symmetric pairing on $\Sigma$, A be its operator with respect to $\mathscr{B}$ and $E \in \operatorname{End}(\Sigma)$ be an endomorphism of $\Sigma$. Then the endomorphism $E_{A} \stackrel{\text { def. }}{=} E \circ A \in \operatorname{End}(\Sigma)$ is $\mathscr{B}^{\prime}$-admissible iff the following relations hold:

$$
E^{t}=\sigma E \text { and } E \circ A \circ E=\operatorname{tr}(E \circ A) E .
$$

Proof Let ${ }^{T}$ denote transposition of endomorphisms with respect to $\mathscr{B}^{\prime}$. By definition, $E_{A}$ is $\mathscr{B}^{\prime}$-admissible if:

$$
E_{A}^{T}=E_{A} \quad \text { and } \quad E_{A}^{2}=\operatorname{tr}\left(E_{A}\right) E_{A} .
$$

Since $A$ is invertible, the second of these conditions amounts to the second relation in (12). On the other hand, we have:

$$
E_{A}^{T}=(E A)^{T}=A^{T} E^{T}=A^{t}=A^{t}\left(A^{-1}\right)^{t} E^{t} A^{t}=E^{t} A^{t}=\sigma E^{t} A,
$$

where we used Proposition 2.14. Hence the first condition in (13) is equivalent with $\sigma E^{t} A=$ $E A$, which in turn amounts to $E^{t}=\sigma E$ since $A$ is invertible.

\subsection{Characterizations of tame admissible endomorphisms}

The following gives an open subset of the cone $\mathcal{C}$ of admissible endomorphisms which consists of rank one elements.

Proposition 2.18 Let $E \in \mathcal{C}(\Sigma, \mathscr{B})$ be a $\mathscr{B}$-admissible endomorphism of $\Sigma$. If $\operatorname{tr}(E) \neq 0$, then $E$ is of rank one.

Proof Define $P \stackrel{\text { def. }}{=} \frac{E}{\operatorname{tr}(E)}$. Then $P^{2}=P$ (which implies $\operatorname{rk}(P)=\operatorname{tr}(P)$ ) and $\operatorname{tr}(P)=1$, whence $\operatorname{rk}(E)=\operatorname{rk}(P)=\operatorname{tr}(P)=1$.

Define

$$
\mathcal{K}_{0} \stackrel{\text { def. }}{=}\{\xi \in \Sigma \mid \mathscr{B}(\xi, \xi)=0\}, \mathcal{K}_{\mu} \stackrel{\text { def. }}{=}\{\xi \in \Sigma \mid \mathscr{B}(\xi, \xi)=\mu\},
$$

where $\mu \in\{-1,+1\}$. When $\mathscr{B}$ is symmetric, the set $\mathcal{K}_{0} \subset \Sigma$ is the isotropic cone of $\mathscr{B}$ and $\mathcal{K}_{\mu}$ are the positive and negative unit "pseudo-spheres" defined by $\mathscr{B}$. When $\mathscr{B}$ is skew-symmetric, we have $\mathcal{K}_{0}=\Sigma$ and $\mathcal{K}_{\mu}=\emptyset$. Lemma 2.6 and Proposition 2.18 imply: 
Corollary 2.19 Assume that $\mathscr{B}$ is symmetric (i.e. $\sigma=+1$ ). For any $\mu \in\{-1,1\}$, the set $\mathcal{E}_{+}\left(\mathcal{K}_{\mu}\right) \cup \mathcal{E}_{-}\left(\mathcal{K}_{\mu}\right)$ is the real algebraic submanifold of End $(\Sigma)$ given by:

$$
\mathcal{E}_{+}\left(\mathcal{K}_{\mu}\right) \cup \mathcal{E}_{-}\left(\mathcal{K}_{\mu}\right)=\left\{E \in \operatorname{End}(\Sigma) \mid E \circ E=\mu E, E^{t}=E, \operatorname{tr}(E)=\mu\right\} .
$$

Proposition 2.20 If $\mathscr{B}$ is a scalar product, then every non-zero $\mathscr{B}$-admissible endomorphism $E \in \mathcal{C} \backslash\{0\}$ is tame, whence $\mathcal{Z}=\mathcal{C}$. In this case, the signature of $E$ with respect to $\mathscr{B}$ is given by:

$$
\kappa_{E}=\operatorname{sign}(\operatorname{tr}(E)) .
$$

Proof Let $E \in \mathcal{C}$. By Proposition 2.18, the first statement follows if we can show that $\operatorname{tr}(E) \neq 0$ when $E \neq 0$. Since $E$ is admissible, it is symmetric with respect to the scalar product $\mathscr{B}$ and hence diagonalizable with eigenvalues $\lambda_{1}, \ldots, \lambda_{N} \in \mathbb{R}$. Taking the trace of equation $E^{2}=\operatorname{tr}(E) E$ gives:

$$
\operatorname{tr}(E)^{2}=\sum_{i=1}^{N} \lambda_{i}^{2} .
$$

Since the right-hand side is a sum of squares, it vanishes iff $\lambda_{1}=\cdots=\lambda_{N}=0$, i.e. iff $E=0$. This proves the first statement. To prove the second statement, recall from Lemma 2.6 that any non-zero tame admissible endomorphism $E$ has the form $E=\kappa_{E} \xi \otimes \xi^{*}$ for some $\xi \in \dot{\Sigma}$. Taking the trace of this relation gives:

$$
\operatorname{tr}(E)=\kappa_{E} \xi^{*}(\xi)=\kappa_{E} \mathscr{B}(\xi, \xi),
$$

which implies (14) since $\mathscr{B}(\xi, \xi)>0$.

A quick inspection of examples shows that there exist nontrivial admissible endomorphisms which are not tame (and thus satisfy $\operatorname{tr}(E)=0$ ) as soon as there exists a totally isotropic subspace of $\Sigma$ of dimension at least two. In these cases we need to impose further conditions on the elements of $\mathcal{C}$ in order to guarantee tameness. To describe such conditions, we consider the more general equation:

$$
E \circ A \circ E=\operatorname{tr}(A \circ E) E \quad \forall A \in \operatorname{End}(\Sigma),
$$

which is automatically satisfied by every $E \in \operatorname{Im}\left(\mathcal{E}_{+}\right) \cup \operatorname{Im}\left(\mathcal{E}_{-}\right)$.

Proposition 2.21 The following statements are equivalent for any endomorphism $E \in \mathcal{C}$ which satisfies the condition $E^{t}=\sigma E$ :

(a) $E$ is $\mathscr{B}$-admissible and $\operatorname{rk}(E)=1$.

(b) We have $E^{2}=\operatorname{tr}(E) E$ and there exists an endomorphism $A \in \operatorname{End}(\Sigma)$ satisfying:

$$
E \circ A \circ E=\operatorname{tr}(E \circ A) E \text { and } \operatorname{tr}(E \circ A) \neq 0 \text {. }
$$

(c) $E \neq 0$ and the relation:

$$
E \circ A \circ E=\operatorname{tr}(E \circ A) E
$$

is holds for every endomorphism $A \in \operatorname{End}(\Sigma)$.

Proof We first prove the implication $(b) \Rightarrow(a)$. By Proposition 2.18, it suffices to consider the case $\operatorname{tr}(E)=0$. Assume $A \in \operatorname{End}(\Sigma)$ satisfies (15). Define:

$$
A_{\epsilon}=\mathrm{Id}+\frac{\epsilon}{\operatorname{tr}(E \circ A)} A \text {, }
$$


where $\epsilon \in \mathbb{R}_{>0}$ is a positive constant. For $\epsilon>0$ small enough, $A_{\epsilon}$ is invertible and the endomorphism $E_{\epsilon} \stackrel{\text { def. }}{=} E \circ A_{\epsilon}$ has non-vanishing trace given by $\operatorname{tr}\left(E_{\epsilon}\right)=\epsilon$. The first relation in (15) gives:

$$
E_{\epsilon} \circ E_{\epsilon}=\epsilon E_{\epsilon} \text {. }
$$

Hence $P \stackrel{\text { def. }}{=} \frac{1}{\epsilon} E_{\epsilon}$ satisfies $P^{2}=P$ and $\operatorname{tr}(P)=1$, whence $\operatorname{rk}\left(E_{\epsilon}\right)=\operatorname{rk}(P)=1$. Since $A_{\epsilon}$ is invertible, this implies $\operatorname{rk}(E)=1$ and hence $(a)$ holds.

The implication $(a) \Rightarrow(c)$ follows directly from Lemma 2.6, which shows that $E \in$ $\operatorname{Im}\left(\mathcal{E}_{\kappa}\right)$ for some sign factor $\kappa$. For the implication $(c) \Rightarrow(b)$, notice first that setting $A=\operatorname{Id}$ in (16) gives $E^{2}=\operatorname{tr}(E)$. Non-degeneracy of the bilinear form induced by the trace on the space $\operatorname{End}(\Sigma)$ now shows that we can choose $A$ in Eq. (16) such that $\operatorname{tr}(E \circ A) \neq 0$.

Proposition 2.22 Let $A$ be a taming of $\mathscr{B}$ and let $\mathscr{B}^{\prime} \stackrel{\text { def. }}{=} \mathscr{B}_{A}$ be the corresponding scalar product on $\Sigma$. Then the following statements are equivalent for any endomorphism $E \in$ $\operatorname{End}(\Sigma)$ :

(a) $E$ is $\mathscr{B}$-admissible and $\operatorname{rk}(E)=1$.

(b) E satisfies $E^{t}=\sigma E$ as well as conditions (15) with respect to A.

In this case, there exists a non-zero vector $\xi \in \Sigma$ such that:

$$
E=\kappa \xi \otimes \xi^{*}=\kappa^{\prime} \xi \otimes \xi^{\vee},
$$

where $\xi^{\vee}=\sigma \xi^{*} \circ$ A denotes the linear functional dual to $\xi$ with respect $\mathscr{B}^{\prime}$ while:

$$
\kappa^{\prime}=\operatorname{sign}(\operatorname{tr}(E \circ A)) \in\{-1,1\}
$$

is the signature of $E \circ A$ with respect to $\mathscr{B}^{\prime}$ and:

$$
\kappa=\sigma \kappa^{\prime}=\sigma \operatorname{sign}(\operatorname{tr}(E \circ A)) \in\{-1,1\}
$$

is the signature of $E$ with respect to $\mathscr{B}$.

Proof Set $E_{A} \stackrel{\text { def. }}{=} E \circ A$. To prove the implication $(a) \Rightarrow(b)$, assume that $E$ is $\mathscr{B}$-admissible and of rank one. Then Proposition 2.21 shows that we have $E \circ A \circ E=\operatorname{tr}(E \circ A) E$. Since $A$ is $\mathscr{B}$-admissible, we also have $E^{t}=\sigma E$. It follows that $E_{A}$ is $\mathscr{B}^{\prime}$-admissible by Proposition 2.17. Since $\mathscr{B}^{\prime}$ is a scalar product, Proposition 2.20 implies that $E_{A}$ is tame and hence $\operatorname{tr}\left(E_{A}\right) \neq 0$ since $E_{A}$ is non-zero. Thus $\operatorname{tr}(E \circ A) \neq 0$. Combining everything, this shows that (b) holds.

To prove the implication $(b) \Rightarrow(a)$, assume that $E$ satisfies $E^{t}=\sigma E$ as well as (15). By Proposition 2.17, this implies that $E_{A}$ is $\mathscr{B}^{\prime}$-admissible. Since $\operatorname{tr}\left(E_{A}\right)=\operatorname{tr}(E \circ A) \neq 0$, Proposition $2.18 \mathrm{implies} \operatorname{rk}\left(E_{A}\right)=1$. Hence $E_{A}$ is nonzero, tame and admissible with respect to the scalar product $\mathscr{B}^{\prime}$. We thus have $E_{A}=\kappa^{\prime} \xi \otimes \xi^{\vee}$, where $\kappa^{\prime}=\operatorname{sign}\left(\operatorname{tr}\left(E_{A}\right)\right)=$ $\operatorname{sign}\left(\operatorname{tr}(E \circ A)\right.$ ) (see Proposition 2.20) and $\xi \in \Sigma$ is a non-zero vector. Here $\xi^{\vee} \in \Sigma^{*}$ is the dual of $\xi$ with respect to $\mathscr{B}^{\prime}$, which is given by:

$$
\xi^{\vee}\left(\xi^{\prime}\right) \stackrel{\text { def. }}{=} \mathscr{B}^{\prime}\left(\xi^{\prime}, \xi\right)=\mathscr{B}\left(\xi^{\prime}, A \xi\right)=\mathscr{B}\left(A^{t} \xi^{\prime}, \xi\right)=\xi^{*}\left(A^{t} \xi^{\prime}\right)=\sigma \xi^{*}\left(A \xi^{\prime}\right),
$$

i.e. $\xi^{\vee}=\sigma \xi^{*} \circ A$. Thus $E \circ A=E_{A}=\sigma \kappa_{A} \xi \otimes \xi^{*} \circ A$ and hence $E=\kappa \xi \otimes \xi^{*}$ (with $\kappa \stackrel{\text { def. }}{=} \sigma \kappa^{\prime}$ ) because $A$ is invertible. Thus $E$ belongs to $\operatorname{Im}\left(\mathcal{E}_{\kappa}\right)$ and hence is $\mathscr{B}$-admissible and of rank one. 
Remark 2.23 When $A$ is a taming of $\mathscr{B}$, Proposition 2.22 shows that conditions (15) and the condition $E^{t}=\sigma E$ automatically imply $E^{2}=\operatorname{tr}(E) A$ and hence tameness of $E$. In this case, Proposition 2.21 shows that $E$ also satisfies $E \circ B \circ E=\operatorname{tr}(E \circ B) E$ for any $B \in \operatorname{End}(\Sigma)$.

Proposition 2.22 gives the following characterization of $\mathbb{P} \mathcal{Z}$.

Corollary 2.24 Let $A$ be a taming of $\mathscr{B}$. Then the image of the projective squaring map $\mathbb{P} \mathcal{E}$ is the real algebraic submanifold of $\mathbb{P}(\operatorname{End}(\Sigma))$ given by:

$\mathbb{P} \mathcal{Z}(\Sigma, \mathscr{B})=\left\{E \in \mathbb{P}(\operatorname{End}(\Sigma)) \mid E \circ E=\operatorname{tr}(E) E, E^{t}=\sigma E, E \circ A \circ E=\operatorname{tr}(E \circ A) E\right\}$.

\subsection{Two-dimensional examples}

Let $\Sigma$ be a two-dimensional $\mathbb{R}$-vector space with basis $\Delta \stackrel{\text { def. }}{=}\left\{e_{i}\right\}_{i=1,2}$. Any vector $\xi \in \Sigma$ expands as:

$$
\xi=\xi_{1} e_{1}+\xi_{2} e_{2} \quad \text { with } \xi_{1}, \xi_{2} \in \mathbb{R} .
$$

Let $E_{\xi} \stackrel{\text { def. }}{=} \mathcal{E}_{\kappa}(\xi) \stackrel{\text { def. }}{=} \kappa \xi \otimes \xi^{*} \in \operatorname{End}(\Sigma)$, where $\kappa \in\{-1,1\}$. For any $S \in \operatorname{End}(\Sigma)$, let $\hat{S}$ denote the matrix of $S$ in the basis $\Delta$.

Example 2.25 Let $\mathscr{B}$ be a scalar product on $\Sigma$ having $\Delta$ as an orthonormal basis. Then:

$$
\hat{E}_{\xi}=\kappa\left(\begin{array}{cc}
\xi_{1}^{2} & \xi_{1} \xi_{2} \\
\xi_{1} \xi_{2} & \xi_{2}^{2}
\end{array}\right)
$$

and the relations $E_{\xi}^{2}=\operatorname{tr}\left(E_{\xi}\right) E_{\xi}$ and $E_{\xi}^{t}=E_{\xi}$ follow from this form. Let $E \in \operatorname{End}(\Sigma)$ satisfy $E^{2}=\operatorname{tr}(E) E$ and $E^{t}=E$. The second of these conditions implies:

$$
\hat{E}=\left(\begin{array}{cc}
k_{1} & b \\
b & k_{2}
\end{array}\right) \quad\left(\text { with } b, k_{1}, k_{2} \in \mathbb{R}\right) .
$$

Condition $E^{2}=\operatorname{tr}(E) E$ amounts to $b^{2}=k_{1} k_{2}$, implying that $k_{1}$ and $k_{2}$ have the same sign unless at least one of them vanishes (in which case $b$ must also vanish). Since $E$ is $\mathscr{B}$-symmetric (and hence diagonalizable), its trace $\operatorname{tr}(E)=k_{1}+k_{2}$ vanishes iff $E=0$. Assume $E \neq 0$ and set:

$$
\kappa \stackrel{\text { def. }}{=} \operatorname{sign}(\operatorname{tr}(E))=\operatorname{sign}\left(k_{1}+k_{2}\right), \quad \xi_{1} \stackrel{\text { def. }}{=} \sqrt{\left|k_{1}\right|}, \quad \xi_{2} \stackrel{\text { def. }}{=} \operatorname{sign}(b) \kappa \sqrt{\left|k_{2}\right|} .
$$

Then $k_{1}=\kappa \xi_{1}^{2}, k_{2}=\kappa \xi_{2}^{2}$ and $b=\kappa \xi_{1} \xi_{2}$, showing that $E=E_{\xi}$ for some $\xi \in \Sigma \backslash\{0\}$. Hence conditions $E^{2}=\operatorname{tr}(E) E$ and $E=E^{t}$ characterize endomorphisms of the form $E_{\xi}$.

Example 2.26 Let $\mathscr{B}$ be a split signature inner product on $\Sigma$ having $\Delta$ as an orthonormal basis:

$$
\mathscr{B}\left(e_{1}, e_{1}\right)=1, \mathscr{B}\left(e_{2}, e_{2}\right)=-1, \mathscr{B}\left(e_{1}, e_{2}\right)=\mathscr{B}\left(e_{2}, e_{1}\right)=0 .
$$

Then $\mathscr{B}$ is tamed by the operator $A$ with matrix $\hat{A}=\operatorname{diag}(+1,-1)$, which corresponds to the scalar product $(-,-)$ defined on $\Sigma$ through:

$$
\left(e_{1}, e_{1}\right)=\left(e_{2}, e_{2}\right)=1, \quad\left(e_{1}, e_{2}\right)=\left(e_{2}, e_{1}\right)=0 .
$$

We have:

$$
\hat{E}_{\xi}=\kappa\left(\begin{array}{cc}
\xi_{1}^{2} & -\xi_{1} \xi_{2} \\
\xi_{1} \xi_{2} & -\xi_{2}^{2}
\end{array}\right)
$$


and the relations $E_{\xi}^{2}=\operatorname{tr}\left(E_{\xi}\right) E_{\xi}$ and $E_{\xi}^{t}=E_{\xi}$ follow directly from this form, where ${ }^{t}$ denotes the adjoint taken with respect to $\mathscr{B}$. Let $E \in \operatorname{End}(\Sigma)$ satisfy $E^{t}=E$. Then:

$$
\hat{E}=\left(\begin{array}{cc}
k_{1} & -b \\
b & k_{2}
\end{array}\right) \quad \text { and } \hat{E} \hat{A}=\left(\begin{array}{cc}
k_{1} & b \\
b & -k_{2}
\end{array}\right) \quad\left(\text { with } b, k_{1}, k_{2} \in \mathbb{R}\right) \text {. }
$$

Direct computation shows that the conditions $E^{2}=\operatorname{tr}(E) E$ and $E \circ A \circ E=\operatorname{tr}(E \circ A) E$ are equivalent to each other in this two-dimensional example and amount to the relation $b^{2}=-k_{1} k_{2}$, which implies that $E$ vanishes iff $k_{1}=k_{2}$. Let us assume that $E \neq 0$ and set:

$$
\kappa \stackrel{\text { def. }}{=} \operatorname{tr}(E \circ A)=\operatorname{sign}\left(k_{1}-k_{2}\right), \quad \xi_{1} \stackrel{\text { def. }}{=} \sqrt{\left|k_{1}\right|}, \quad \xi_{2} \stackrel{\text { def. }}{=} \operatorname{sign}(b) \kappa \sqrt{\left|k_{2}\right|},
$$

where $\operatorname{sign}(b) \stackrel{\text { def. }}{=} 0$ if $b=0$. Then it is easy to see that $k_{1}=\kappa \xi_{1}^{2}, k_{2}=-\kappa \xi_{2}^{2}$ and $b=\kappa \xi_{1} \xi_{2}$, which implies $E=E_{\xi}$. In this example endomorphisms $E$ that can be written in the form $E_{\xi}$ are characterized by the condition $E^{t}=E$, together with either of the two equivalent conditions $E^{2}=\operatorname{tr}(E) E$ or $E \circ A \circ E=\operatorname{tr}(E \circ A) E$. Notice that $\operatorname{tr}(E)=k_{1}+k_{2}$ can vanish in this case. However, in this low-dimensional example, the conditions $E \circ E=\operatorname{tr}(E) E$ and $E^{t}=E$ suffice to characterize endomorphisms of the form $E_{\xi}$, including those which satisfy $\operatorname{tr}(E)=0$.

Example 2.27 Let $\mathscr{B}$ a symplectic pairing on $\Sigma$ having $\Delta$ as a Darboux basis:

$$
\mathscr{B}\left(e_{1}, e_{1}\right)=\mathscr{B}\left(e_{2}, e_{2}\right)=0, \mathscr{B}\left(e_{1}, e_{2}\right)=-\mathscr{B}\left(e_{2}, e_{1}\right)=1 .
$$

The complex structure $A$ of $\Sigma$ with matrix given by:

$$
\hat{A}=\left(\begin{array}{cc}
0 & 1 \\
-1 & 0
\end{array}\right)
$$

tames $\mathscr{B}$ to the scalar product $(-,-)$ defined through:

$$
\left(e_{1}, e_{1}\right)=\left(e_{2}, e_{2}\right)=1, \quad\left(e_{1}, e_{2}\right)=\left(e_{2}, e_{1}\right)=0 .
$$

We have:

$$
\hat{E}_{\xi}=\kappa\left(\begin{array}{cc}
\xi_{1} \xi_{2} & -\xi_{1}^{2} \\
\xi_{2}^{2} & -\xi_{1} \xi_{2}
\end{array}\right)
$$

which implies $E_{\xi}^{2}=0$ and $E_{\xi}^{t}=-E_{\xi}$, where ${ }^{t}$ denotes transposition with respect to $\mathscr{B}$. Let $E \in \operatorname{End}(\Sigma)$ be an endomorphism satisfying $E^{t}=-E$. This condition implies:

$$
\hat{E}=\left(\begin{array}{ll}
k & -b \\
c & -k
\end{array}\right), \quad \hat{E} \hat{A}=\left(\begin{array}{ll}
b & k \\
k & c
\end{array}\right) \quad \text { (with } k, b, c \in \mathbb{R} \text { ). }
$$

Notice that $\operatorname{tr}(E)=0$. Direct computation shows that the conditions $E^{2}=0$ and $E \circ A \circ E=$ $\operatorname{tr}(E \circ A) E$ are equivalent to each other in this two-dimensional example and amount to the relation $k^{2}=b c$, which in particular shows that $E$ vanishes iff $b=-c$. Assume that $E \neq 0$ and set:

$$
\kappa \stackrel{\text { def. }}{=} \operatorname{tr}(E \circ A)=\operatorname{sign}(b+c), \quad \xi_{1} \stackrel{\text { def. }}{=} \sqrt{|b|}, \quad \xi_{2} \stackrel{\text { def. }}{=} \operatorname{sign}(k) \kappa \sqrt{|c|},
$$

where $\operatorname{sign}(k) \stackrel{\text { def. }}{=} 0$ if $k=0$. Then it is easy to see that $b=\kappa \xi_{1}^{2}, c=\kappa \xi_{2}^{2}$ and $k=\kappa \xi_{1} \xi_{2}$, which shows that $E=E_{\xi}$. Hence endomorphism which can be written in this form are characterized by the condition $E^{t}=-E$ together with either of the conditions $E^{2}=0$ or $E \circ A \circ E=\operatorname{tr}(E \circ A) E$, which are equivalent to each other in this low-dimensional example. 


\subsection{Including linear constraints}

The following result will be used in Sects. 3 and 4 .

Proposition 2.28 Let $Q \in \operatorname{End}(\Sigma)$ and $\kappa \in\{-1,1\}$ be a fixed sign factor. A real spinor $\xi \in \Sigma$ satisfies $Q(\xi)=0$ if and only if $Q \circ \mathcal{E}_{\kappa}(\xi)=0$ or, equivalently, $\mathcal{E}_{\kappa}(\xi) \circ Q^{t}=0$, where $Q^{t}$ is the adjoint of $Q$ with respect to $\mathscr{B}$.

Proof Take $\xi \in \Sigma$ and assume $Q(\xi)=0$. Then:

$$
\left(Q \circ \mathcal{E}_{\kappa}(\xi)\right)(\chi)=\kappa Q(\xi) \xi^{*}(\chi)=0 \quad \forall \chi \in \Sigma
$$

and hence $Q \circ \mathcal{E}_{\kappa}(\xi)=0$. Conversely, assume that $Q \circ \mathcal{E}_{\kappa}(\xi)=0$ and pick $\chi \in \Sigma$ such that $\xi^{*}(\chi) \neq 0$ (which is possible since $\mathscr{B}$ is non-degenerate). Then the same calculation as before gives:

$$
Q(\xi) \xi^{*}(\chi)=0,
$$

implying $Q(\xi)=0$. The statement for $Q^{t}$ follows from the fact that $\mathscr{B}$-transposition is an anti-automorphism of the $\mathbb{R}$-algebra $(\operatorname{End}(\Sigma), \circ)$, upon noticing that the relation $\mathcal{E}_{K}(\xi)^{t}=$ $\sigma \mathcal{E}_{\kappa}(\xi)$ implies $\left(Q \circ \mathcal{E}_{\kappa}(\xi)\right)^{t}=\sigma \mathcal{E}_{\kappa}(\xi) \circ Q^{t}$.

Example 2.29 Let $(\Sigma, \mathscr{B})$ be a two-dimensional Euclidean vector space with orthonormal basis $\Delta$ as in Example 2.25. Let $Q \in \operatorname{End}(\Sigma)$ have matrix:

$$
\hat{Q}=\left(\begin{array}{ll}
q & 0 \\
0 & 0
\end{array}\right) \quad\left(\text { with } q \in \mathbb{R}^{\times}\right)
$$

in this basis. Given $\xi \in \Sigma$, Example 2.25 gives:

$$
\hat{E}_{\xi}=\kappa\left(\begin{array}{cc}
\xi_{1}^{2} & \xi_{1} \xi_{2} \\
\xi_{1} \xi_{2} & \xi_{2}^{2}
\end{array}\right), \quad \hat{Q} \hat{E}_{\xi}=\kappa\left(\begin{array}{cc}
\xi_{1}^{2} q & q \xi_{1} \xi_{2} \\
0 & 0
\end{array}\right)
$$

Thus $Q \circ E_{\xi}$ vanishes iff $\xi_{1}=0$, i.e. iff $Q(\xi)=0$.

\section{From real spinors to polyforms}

\subsection{Admissible pairings for irreducible real Clifford modules}

Let $V$ be an oriented $d$-dimensional $\mathbb{R}$-vector space equipped with a non-degenerate metric $h$ of signature $p-q \equiv_{8} 0,2$ (hence the dimension $d=p+q$ of $V$ is even) and let $\left(V^{*}, h^{*}\right)$ be the quadratic space dual to $(V, h)$, where $h^{*}$ denotes the metric dual to $h$. Let $\operatorname{Cl}\left(V^{*}, h^{*}\right)$ be the real Clifford algebra of this dual quadratic space, viewed as a $\mathbb{Z}_{2}$-graded associative algebra with decomposition:

$$
\mathrm{Cl}\left(V^{*}, h^{*}\right)=\mathrm{Cl}^{\mathrm{ev}}\left(V^{*}, h^{*}\right) \oplus \mathrm{Cl}^{\mathrm{odd}}\left(V^{*}, h^{*}\right) .
$$

In our conventions, the Clifford algebra satisfies (notice the sign !):

$$
\theta^{2}=+h^{*}(\theta, \theta) \quad \forall \theta \in V^{*} .
$$

Let $\pi$ denote the standard automorphism of $\mathrm{Cl}\left(V^{*}, h^{*}\right)$, which acts as minus the identity on $V^{*} \subset \mathrm{Cl}\left(V^{*}, h^{*}\right)$ and $\tau$ denote the standard anti-automorphism, which acts as the identity on $V^{*} \subset \mathrm{Cl}\left(V^{*}, h^{*}\right)$. These two commute and their composition is an anti-automorphism 
denoted by $\hat{\tau}=\pi \circ \tau=\tau \circ \pi$. Let $\mathrm{Cl}^{\times}\left(V^{*}, h^{*}\right)$ denote the group of units $\mathrm{Cl}\left(V^{*}, h^{*}\right)$. Its twisted adjoint action is the morphism of groups $\widehat{\mathrm{Ad}}: \mathrm{Cl}^{\times}\left(V^{*}, h^{*}\right) \rightarrow \operatorname{Aut}\left(\mathrm{Cl}\left(V^{*}, h^{*}\right)\right)$ defined through:

$$
\widehat{\operatorname{Ad}}_{x}(y)=\pi(x) y x^{-1} \quad \forall x, y \in \mathrm{Cl}^{\times}\left(V^{*}, h^{*}\right) .
$$

We denote by $\mathbb{\Gamma}\left(V^{*}, h^{*}\right) \subset \mathrm{Cl}\left(V^{*}, h^{*}\right)$ the Clifford group, which is defined as follows:

$$
\mathbb{\Gamma}\left(V^{*}, h^{*}\right) \stackrel{\text { def. }}{=}\left\{x \in \mathrm{Cl}^{\times}\left(V^{*}, h^{*}\right) \mid \widehat{\operatorname{Ad}}_{x}\left(V^{*}\right)=V^{*}\right\},
$$

This fits into the short exact sequence:

$$
1 \rightarrow \mathbb{R}^{\times} \hookrightarrow \mathbb{\Gamma}\left(V^{*}, h^{*}\right) \stackrel{\widehat{\mathrm{Ad}}}{\longrightarrow} \mathrm{O}\left(V^{*}, h^{*}\right) \rightarrow 1,
$$

where $\mathrm{O}\left(V^{*}, h^{*}\right)$ is the orthogonal group of the quadratic space $\left(V^{*}, h^{*}\right)$. Recall that the pin and spin groups of $\left(V^{*}, h^{*}\right)$ are the subgroups of $\mathbb{T}\left(V^{*}, h^{*}\right)$ defined through:

$$
\begin{aligned}
& \operatorname{Pin}\left(V^{*}, h^{*}\right) \stackrel{\text { def. }}{=}\left\{x \in \mathbb{\Gamma}\left(V^{*}, h^{*}\right) \mid N(x)^{2}=1\right\}, \\
& \operatorname{Spin}\left(V^{*}, h^{*}\right) \stackrel{\text { def. }}{=} \operatorname{Pin}\left(V^{*}, h^{*}\right) \cap \mathrm{Cl}^{\mathrm{ev}}\left(V^{*}, h^{*}\right),
\end{aligned}
$$

where $N: \mathbb{\Gamma}\left(V^{*}, h^{*}\right) \rightarrow \mathbb{R}^{\times}$is the Clifford norm morphism, which is given by:

$$
N(x) \stackrel{\text { def. }}{=} \hat{\tau}(x) x \quad \forall x \in \mathbb{\Gamma}\left(V^{*}, h^{*}\right) .
$$

We have $N(x)^{2}=N(\pi(x))^{2}$ for all $x \in \mathbb{\Gamma}\left(V^{*}, h^{*}\right)$. For $p q \neq 0$, the groups $\operatorname{SO}\left(V^{*}, h^{*}\right)$, $\operatorname{Spin}\left(V^{*}, h^{*}\right)$ and $\operatorname{Pin}\left(V^{*}, h^{*}\right)$ are disconnected; the first have two connected components while the last has four. The connected components of the identity in $\operatorname{Spin}\left(V^{*}, h^{*}\right)$ and $\operatorname{Pin}\left(V^{*}, h^{*}\right)$ coincide, being given by:

$$
\operatorname{Spin}_{0}\left(V^{*}, h^{*}\right)=\left\{x \in \mathbb{\Gamma}\left(V^{*}, h^{*}\right) \mid N(x)=1\right\}
$$

and we have $\operatorname{Spin}\left(V^{*}, h^{*}\right) / \operatorname{Spin}_{0}\left(V^{*}, h^{*}\right) \simeq \mathbb{Z}_{2}$ and $\operatorname{Pin}\left(V^{*}, h^{*}\right) / \operatorname{Spin}_{0}\left(V^{*}, h^{*}\right) \simeq \mathbb{Z}_{2} \times \mathbb{Z}_{2}$.

Let $\Sigma$ be a finite-dimensional $\mathbb{R}$-vector space and $\gamma: \mathrm{Cl}\left(V^{*}, h^{*}\right) \rightarrow \operatorname{End}(\Sigma)$ a Clifford representation. Then $\operatorname{Spin}\left(V^{*}, h^{*}\right)$ acts on $\Sigma$ through the restriction of $\gamma$ and (20) induces the short exact sequence:

$$
1 \rightarrow \mathbb{Z}_{2} \rightarrow \operatorname{Spin}\left(V^{*}, h^{*}\right) \stackrel{\widehat{\operatorname{Ad}}}{\longrightarrow} \mathrm{SO}\left(V^{*}, h^{*}\right) \rightarrow 1,
$$

which in turn gives the exact sequence:

$$
1 \rightarrow \mathbb{Z}_{2} \rightarrow \operatorname{Spin}_{0}\left(V^{*}, h^{*}\right) \stackrel{\widehat{\operatorname{Ad}}}{\longrightarrow} \operatorname{SO}_{0}\left(V^{*}, h^{*}\right) \rightarrow 1 .
$$

Here $\mathrm{SO}_{0}\left(V^{*}, h^{*}\right)$ denotes the connected component of the identity of the special orthogonal group $\mathrm{SO}\left(V^{*}, h^{*}\right)$. In signatures $p-q \equiv_{8} 0,2$ (the "real/normal simple case" of [70]), the algebra $\mathrm{Cl}\left(V^{*}, h^{*}\right)$ is simple and isomorphic (as a unital associative $\mathbb{R}$-algebra) to the algebra of square real matrices of size $N=2^{\frac{d}{2}}$. In such signatures $\mathrm{Cl}\left(V^{*}, h^{*}\right)$ admits a unique irreducible real left module $\Sigma$, which has dimension $N$. This irreducible representation is faithful and surjective, hence in such signatures the representation map $\gamma: \operatorname{Cl}\left(V^{*}, h^{*}\right) \stackrel{\simeq}{\rightarrow}$ $\operatorname{End}(\Sigma)$ is an isomorphism of unital $\mathbb{R}$-algebras.

We will equip $\Sigma$ with a non-degenerate bilinear pairing which is compatible with Clifford multiplication. Ideally, such compatibility should translate into invariance under the natural action of the pin group. However, this condition cannot be satisfied when if $p q \neq 0$. Instead, we consider the weaker notion of admissible bilinear pairing introduced in [4,5] (see [63,66, 
70] for applications to supergravity), which encodes the best compatibility condition with Clifford multiplication that can be imposed on a bilinear pairing on $\Sigma$ in arbitrary dimension and signature. The following result of [56] summarizes the main properties of admissible bilinear pairings.

Theorem 3.1 [56, Theorem 13.17] Suppose that h has signature $p-q \equiv_{8} 0,2$. Then the irreducible real Clifford module $\Sigma$ admits two non-degenerate bilinear pairings $\mathscr{B}_{+}: \Sigma \times$ $\Sigma \rightarrow \mathbb{R}$ and $\mathscr{B}_{-}: \Sigma \times \Sigma \rightarrow \mathbb{R}$ (each determined up to multiplication by a non-zero real number) such that:

$$
\begin{aligned}
& \mathscr{B}_{+}\left(\gamma(x)\left(\xi_{1}\right), \xi_{2}\right)=\mathscr{B}_{+}\left(\xi_{1}, \gamma(\tau(x))\left(\xi_{2}\right)\right), \\
& \mathscr{B}_{-}\left(\gamma(x)\left(\xi_{1}\right), \xi_{2}\right)=\mathscr{B}_{-}\left(\xi_{1}, \gamma(\hat{\tau}(x))\left(\xi_{2}\right)\right),
\end{aligned}
$$

for all $x \in C l\left(V^{*}, h^{*}\right)$ and $\xi_{1}, \xi_{2} \in \Sigma$. The symmetry properties of $\mathscr{B}_{+}$and $\mathscr{B}$ - are as follows in terms of the modulo 4 reduction of $k \stackrel{\text { def. }}{=} \frac{d}{2}$ :

\begin{tabular}{lllll}
\hline$k \bmod 4$ & 0 & 1 & 2 & 3 \\
\hline $\mathscr{B}_{+}$ & Symmetric & Symmetric & Skew-symmetric & $\begin{array}{l}\text { Skew-symmetric } \\
\mathscr{B}_{-}\end{array}$ \\
\hline
\end{tabular}

In addition, if $\mathscr{B}_{s}$ (with $s \in\{-1,1\}$ ) is symmetric, then it is of split signature unless $p q=0$, in which case $\mathscr{B}_{s}$ is definite.

Definition 3.2 The sign factor $s$ appearing in the previous theorem is called the adjoint type of $\mathscr{B}_{s}$, hence $\mathscr{B}_{+}$is of positive adjoint type $(s=+1)$ and $\mathscr{B}_{-}$is of negative adjoint type $(s=-1)$.

Relations (22) can be written as:

$$
\gamma(x)^{t}=\gamma\left(\left(\pi^{\frac{1-s}{2}} \circ \tau\right)(x)\right) \quad \forall x \in \mathrm{Cl}\left(V^{*}, h^{*}\right),
$$

where ${ }^{t}$ denotes the $\mathscr{B}_{s}$-adjoint. The symmetry type of an admissible bilinear form $\mathscr{B}$ will be denoted by $\sigma \in\{-1,1\}$. If $\sigma=+1$ then $\mathscr{B}$ is symmetric whereas if $\sigma=-1$ then $\mathscr{B}$ is skew-symmetric. Notice that $\sigma$ depends both on $s$ and on the mod 4 reduction of $\frac{d}{2}$.

Definition $3.3 \mathrm{~A}$ (real) paired simple Clifford module for $\left(V^{*}, h^{*}\right)$ is a triplet $\Sigma=(\Sigma, \gamma, \mathscr{B})$, where $(\Sigma, \gamma)$ is a simple $\mathrm{Cl}\left(V^{*}, h^{*}\right)$-module and $\mathscr{B}$ is an admissible pairing on $(\Sigma, \gamma)$. We say that $\Sigma$ has adjoint type $s \in\{-1,1\}$ and symmetry type $\sigma\{-1,1\}$ if $\mathscr{B}$ has these adjoint and symmetry types.

Remark 3.4 Admissible bilinear pairings of positive and negative adjoint types are related through the pseudo-Riemannian volume form $v$ of $\left(V^{*}, h^{*}\right)$ :

$$
\mathscr{B}_{+}=C \mathscr{B}_{-} \circ(\gamma(v) \otimes \mathrm{Id}),
$$

for an appropriate non-zero real constant $C$. For specific applications, we will choose to work with $\mathscr{B}+$ or with $\mathscr{B}_{-}$depending on which admissible pairing yields the computationally simplest polyform associated to a given spinor $\xi \in \Sigma$. When $p q=0$, we will take $\mathscr{B}_{s}$ to be positive-definite (which we can always achieve by rescaling it with a non-zero constant of appropriate sign). See [70] for a useful discussion of properties of admissible pairings in various dimensions and signatures. 
Remark 3.5 Directly from their definition, the pairings $\mathscr{B}_{s}$ satisfy:

$$
\mathscr{B}_{s}\left(\gamma\left(\pi^{\frac{1+s}{2}}(x)\right)\left(\xi_{1}\right), \gamma(x)\left(\xi_{2}\right)\right)=N(x) \mathscr{B}_{s}\left(\xi_{1}, \xi_{2}\right) \quad \forall x \in \mathrm{Cl}\left(V^{*}, h^{*}\right) \forall \xi_{1}, \xi_{2} \in \Sigma .
$$

This relation yields:

$$
\mathscr{B}_{S}\left(\gamma(x)\left(\xi_{1}\right), \xi_{2}\right)+\mathscr{B}_{s}\left(\xi_{1}, \gamma(x)\left(\xi_{2}\right)\right)=0 \quad \forall \xi_{1}, \xi_{2} \in \Sigma
$$

for all $x=\theta_{1} \cdot \theta_{2}$ with $h^{*}$-orthogonal $\theta_{1}, \theta_{2} \in V^{*}$. This implies that $\mathscr{B}_{s}$ is invariant under the action of $\operatorname{Spin}\left(V^{*}, h^{*}\right)$ and hence also under $\operatorname{Spin}_{0}\left(V^{*}, h^{*}\right)$. If $h$ is positive-definite, then $\mathscr{B}+$ is $\operatorname{Pin}\left(V^{*}, h^{*}\right)$-invariant, since it satisfies:

$$
\mathscr{B}_{+}\left(\gamma(\theta)\left(\xi_{1}\right), \gamma(\theta)\left(\xi_{2}\right)\right)=\mathscr{B}_{+}\left(\xi_{1}, \xi_{2}\right) \quad \forall \xi_{1}, \xi_{2} \in \Sigma
$$

for all $\theta \in V^{*}$ of unit norm. If $h$ is negative-definite, then $\mathscr{B}_{-}$is $\operatorname{Pin}\left(V^{*}, h^{*}\right)$-invariant.

For completeness, let us give an explicit construction of $\mathscr{B}_{+}$and $\mathscr{B}_{-}$. Pick an $h^{*}$ orthonormal basis $\left\{e^{i}\right\}_{i=1, \ldots, d}$ of $V^{*}$ and let:

$$
\mathrm{K}\left(\left\{e^{i}\right\}\right) \stackrel{\text { def. }}{=}\{1\} \cup\left\{ \pm e^{i_{1}} \cdots \cdots e^{i_{k}} \mid 1 \leq i_{1}<\cdots<i_{k} \leq d, 1 \leq k \leq d\right\}
$$

be the finite multiplicative subgroup of $\mathrm{Cl}\left(V^{*}, h^{*}\right)$ generated by the elements $\pm e^{i}$. Averaging over $\mathrm{K}\left(\left\{e^{i}\right\}\right)$, we construct an auxiliary positive-definite inner product $(-,-)$ on $\Sigma$ which is invariant under the action of this group. This product satisfies:

$$
\left(\gamma(x)\left(\xi_{1}\right), \gamma(x)\left(\xi_{2}\right)\right)=\left(\xi_{1}, \xi_{2}\right) \quad \forall x \in \mathrm{K}\left(\left\{e^{i}\right\}\right) \quad \forall \xi_{1}, \xi_{2} \in \Sigma .
$$

Write $V^{*}=V_{+}^{*} \oplus V_{-}^{*}$, where $V_{+}^{*}$ is a $p$-dimensional subspace of $V^{*}$ on which $h^{*}$ is positive definite and $V_{-}^{*}$ is a $q$-dimensional subspace of $V^{*}$ on which $h^{*}$ is negative-definite. Fix an orientation on $V_{+}^{*}$ (which induces a unique orientation on $V_{-}^{*}$ compatible with the orientation of $V^{*}$ induced from that of $V$ ) and denote by $\nu_{+}$and $\nu_{-}$the corresponding pseudo-Riemannian volume forms. We have $v=v_{+} \wedge v_{-}$. For $p$ (and hence $q$ ) odd, define:

$$
\mathscr{B}_{ \pm}\left(\xi_{1}, \xi_{2}\right)=\left(\gamma\left(v_{ \pm}\right)\left(\xi_{1}\right), \xi_{2}\right) \quad \forall \xi_{1}, \xi_{2} \in \Sigma
$$

whereas for $p$ (and hence $q$ ) even, set:

$$
\mathscr{B}_{ \pm}\left(\xi_{1}, \xi_{2}\right)=\left(\gamma\left(v_{\mp}\right)\left(\xi_{1}\right), \xi_{2}\right) \quad \forall \xi_{1}, \xi_{2} \in \Sigma .
$$

Then $\mathscr{B}_{ \pm}$are admissible pairings in the sense of Theorem 3.1. Direct computation using Eqs. (25) and (26) gives the following result, which fixes the constant $C$ appearing in Remark 3.4.

Proposition 3.6 The admissible pairings $\mathscr{B}+$ and $\mathscr{B}$ - constructed above are related as follows:

$$
\mathscr{B}_{+}=(-1)^{\left[\frac{q}{2}\right]} \mathscr{B}_{-}(\gamma(v) \otimes I d) .
$$

Thus we can normalize $\mathscr{B}_{ \pm}$such that the constant in $(24)$ is given by $C=(-1)^{\left[\frac{q}{2}\right]}$.

Proposition 3.7 Let $\mathscr{B}$ be an admissible pairing on the real simple $C l\left(V^{*}, h^{*}\right)$-module $(\Sigma, \gamma)$. Then $\mathscr{B}$ is invariant under the action of the group $\operatorname{Spin}_{0}\left(V^{*}, h^{*}\right)$ on $\Sigma$ obtained by restricting $\gamma$. 
Proof We have to show the relation:

$$
\gamma(x)^{t} \circ \gamma(x)=\operatorname{Id} \quad \forall x \in \operatorname{Spin}_{0}\left(V^{*}, h^{*}\right) .
$$

Consider orthonormal basis $\left\{e^{i}\right\}_{i=1, \ldots, d}$ of $\left(V^{*}, h^{*}\right)$ such that $h^{*}\left(e^{i}, e^{i}\right)=+1$ for $i=$ $1, \ldots, p$ and $h^{*}\left(e^{i}, e^{i}\right)=-1$ for $i=p+1, \ldots, d$. A simple computation using relation (23) shows that (28) holds for $x$ of the form $e^{i_{1}} \cdots \cdots e^{i_{2 k}} \cdot e^{j_{1}} \cdots \cdots e^{j_{2 l}}$, where $1 \leq i_{1} \leq \cdots \leq i_{2 k} \leq p$ and $p+1 \leq j_{1} \leq \cdots \leq j_{2 l} \leq d$ with $0 \leq 2 k \leq p$ and $0 \leq 2 l \leq q$. Since such elements generate $\operatorname{Spin}_{0}\left(V^{*}, h^{*}\right)$, we conclude.

\subsection{The Kähler-Atiyah model of $\mathrm{Cl}\left(V^{*}, h^{*}\right)$}

To identify spinors with polyforms, we will use an explicit realization of $\mathrm{Cl}\left(V^{*}, h^{*}\right)$ as a deformation of the exterior $\wedge V^{*}$. This model (which goes back to the work of Chevalley and Riesz $[20,21,83]$ ) has an interpretation as a deformation quantization of the odd symplectic vector space obtained by parity change from the quadratic space $(V, h)$ (see $[12,86])$. It can be constructed using the symbol map and its inverse, the quantization map. Consider first the linear map $\mathfrak{f}: V^{*} \rightarrow \operatorname{End}\left(\wedge V^{*}\right)$ given by:

$$
\mathfrak{f}(\theta)(\alpha)=\theta \wedge \alpha+\iota_{\theta} \alpha \quad \forall \theta \in V^{*} \forall \alpha \in \wedge V^{*} .
$$

We have:

$$
\mathfrak{f}(\theta) \circ \mathfrak{f}(\theta)=h^{*}(\theta, \theta) \quad \forall \theta \in V^{*} .
$$

By the universal property of Clifford algebras, it follows that $f$ extends uniquely to a morphism $\mathfrak{f}: \mathrm{Cl}\left(V^{*}, h^{*}\right) \rightarrow \operatorname{End}\left(\wedge V^{*}\right)$ of unital associative algebras such that $\mathfrak{f} \circ i=\mathfrak{f}$, where $i: V^{*} \hookrightarrow$ $\mathrm{Cl}\left(V^{*}, h^{*}\right)$ is the canonical inclusion of $V$ in $\mathrm{Cl}\left(V^{*}, h^{*}\right)$.

Definition 3.8 The symbol (or Chevalley-Riesz) map is the linear map $\mathfrak{l}: \mathrm{Cl}\left(V^{*}, h^{*}\right) \rightarrow \wedge V^{*}$ defined through:

$$
\mathfrak{l}(x)=\mathfrak{f}(x)(1) \quad \forall x \in \mathrm{Cl}\left(V^{*}, h^{*}\right),
$$

where $1 \in \mathbb{R}$ is viewed as an element of $\wedge^{0}\left(V^{*}\right)=\mathbb{R}$.

The symbol map is an isomorphism of filtered vector spaces. We have:

$$
\mathfrak{l}(1)=1, \mathfrak{l}(\theta)=\theta, \mathfrak{l}\left(\theta_{1} \cdot \theta_{2}\right)=\theta_{1} \wedge \theta_{2}+h^{*}\left(\theta_{1}, \theta_{2}\right) \quad \forall \theta, \theta_{1}, \theta_{2} \in V^{*} .
$$

As expected, $l$ is not a morphism of algebras. The inverse:

$$
\Psi \stackrel{\text { def. }}{=} \mathfrak{l}^{-1}: \wedge V^{*} \rightarrow \mathrm{Cl}\left(V^{*}, h^{*}\right) .
$$

of $\mathfrak{r}$ (called the quantization map) allows one to view $\mathrm{Cl}\left(V^{*}, h^{*}\right)$ as a deformation of the exterior algebra $\left(\wedge V^{*}, \wedge\right)$ (see [12,86]). Using $\mathfrak{l}$ and $\Psi$, we transport the algebra product of $\mathrm{Cl}\left(V^{*}, h^{*}\right)$ to an $h$-dependent unital associative product defined on $\wedge V^{*}$, which deforms the wedge product.

Definition 3.9 The geometric product $\diamond: \wedge V^{*} \times \wedge V^{*} \rightarrow \wedge V^{*}$ is defined through:

$$
\alpha_{1} \diamond \alpha_{2} \stackrel{\text { def. }}{=} \mathfrak{l}\left(\Psi\left(\alpha_{1}\right) \cdot \Psi\left(\alpha_{2}\right)\right) \quad \forall \alpha_{1}, \alpha_{2} \in \wedge V^{*},
$$

where $\cdot$ denotes multiplication in $\mathrm{Cl}\left(V^{*}, h^{*}\right)$. 
By definition, the map $\Psi$ is an isomorphism of unital associative $\mathbb{R}$-algebras ${ }^{3}$ from $\left(\wedge V^{*}, \diamond\right)$ to $\mathrm{Cl}\left(V^{*}, h^{*}\right)$. Through this isomorphism, the inclusion $V^{*} \hookrightarrow \mathrm{Cl}\left(V^{*}, h^{*}\right)$ corresponds to the natural inclusion $V^{*} \hookrightarrow \wedge V^{*}$. We shall refer to $\left(\wedge V^{*}, \diamond\right)$ as the Kähler-Atiyah algebra of the quadratic space $(V, h)$ (see $[47,61])$. It is easy to see that the geometric product satisfies:

$$
\theta \diamond \alpha=\theta \wedge \alpha+\iota_{\theta^{\sharp}} \alpha \quad \forall \theta \in V^{*} \forall \alpha \in \wedge V^{*} .
$$

Also notice the relation:

$$
\theta \diamond \theta=h^{*}(\theta, \theta) \quad \forall \theta \in V^{*} .
$$

The maps $\pi$ and $\tau$ transfer through $\Psi$ to the Kähler-Atiyah algebra, producing unital (anti)automorphisms of the latter which we denote by the same symbols. With this notation, we have:

$$
\pi \circ \Psi=\Psi \circ \pi, \tau \circ \Psi=\Psi \circ \tau .
$$

For any orthonormal basis $\left\{e^{i}\right\}_{i=1, \ldots, d}$ of $V^{*}$ and any $k \in\{1, \ldots, d\}$, we have $e^{1} \diamond \cdots \diamond e^{k}=$ $e^{1} \wedge \cdots \wedge e^{k}$ and:

$$
\pi\left(e^{1} \wedge \cdots \wedge e^{k}\right)=(-1)^{k} e^{1} \wedge \cdots \wedge e^{k}, \quad \tau\left(e^{1} \wedge \cdots \wedge e^{k}\right)=e^{k} \wedge \cdots \wedge e^{1} .
$$

Let $\mathcal{T}\left(V^{*}\right)$ denote the tensor algebra of the (parity change of) $V^{*}$, viewed as a $\mathbb{Z}$-graded associative superalgebra whose $\mathbb{Z}_{2}$-grading is the reduction of the natural $\mathbb{Z}$-grading; thus elements of $V$ have integer degree one and they are odd. Let:

$$
\operatorname{Der}\left(\mathcal{T}\left(V^{*}\right)\right) \stackrel{\text { def. }}{=} \bigoplus_{k \in \mathbb{Z}} \operatorname{Der}^{k}\left(\mathcal{T}\left(V^{*}\right)\right)
$$

denote the $\mathbb{Z}$-graded Lie superalgebra of all superderivations. The minus one integer degree component $\operatorname{Der}^{-1}\left(\mathcal{T}\left(V^{*}\right)\right)$ is linearly isomorphic with the space $\operatorname{Hom}\left(V^{*}, \mathbb{R}\right)=V$ acting by contractions:

$$
\iota_{v}\left(\theta_{1} \otimes \cdots \otimes \theta_{k}\right)=\sum_{i=1}^{k}(-1)^{i-1} \theta_{1} \otimes \cdots \otimes \iota_{v} \theta_{i} \otimes \cdots \otimes \theta_{k} \quad \forall v \in V \quad \forall \theta_{1}, \ldots, \theta_{k} \in V^{*},
$$

while the zero integer degree component $\operatorname{Der}^{0}\left(\mathcal{T}\left(V^{*}\right)\right)=\operatorname{End}\left(V^{*}\right)=\mathfrak{g l}\left(V^{*}\right)$ acts through:

$$
L_{A}\left(\theta_{1} \otimes \cdots \otimes \theta_{k}\right)=\sum_{i=1}^{k} \theta_{1} \otimes \cdots \otimes A\left(\theta_{i}\right) \otimes \cdots \otimes \theta_{k} \quad \forall A \in \mathfrak{g l}\left(V^{*}\right) .
$$

We have an isomorphism of super-Lie algebras:

$$
\operatorname{Der}^{-1}\left(\mathcal{T}\left(V^{*}\right)\right) \oplus \operatorname{Der}^{0}\left(\mathcal{T}\left(V^{*}\right)\right) \simeq V \rtimes \mathfrak{g l}\left(V^{*}\right) .
$$

The action of this super Lie algebra preserves the ideal used to define the exterior algebra as a quotient of $\mathcal{T}\left(V^{*}\right)$ and hence descends to a morphism of super Lie algebras $\mathcal{L}_{\Lambda}: V \rtimes$ $\mathfrak{g l}\left(V^{*}\right) \rightarrow \operatorname{Der}\left(\wedge V^{*}, \wedge\right)$. Contractions also preserve the ideal used to define the Clifford

\footnotetext{
${ }^{3}$ Notice that the geometric product is not compatible with the grading of $\wedge V^{*}$ given by form rank, but only with its mod 2 reduction, because the quantization map does not preserve $\mathbb{Z}$-gradings. Hence the KählerAtiyah algebra is not isomorphic with $\mathrm{Cl}\left(V^{*}, h^{*}\right)$ in the category of Clifford algebras defined in [69]. As such, it provides a different viewpoint on spin geometry, which is particularly useful for our purpose (see $[63,66,70,71])$.
} 
algebra as a quotient of $\mathcal{T}\left(V^{*}\right)$. On the other hand, endomorphisms $A$ of $V^{*}$ preserve that ideal iff $A \in \mathfrak{s o}\left(V^{*}, h^{*}\right)$. Together with contractions, they induce a morphism of super Lie algebras $\mathcal{L}_{\mathrm{Cl}}: V \rtimes \mathfrak{s o}\left(V^{*}, h^{*}\right) \rightarrow \operatorname{Der}\left(\mathrm{Cl}\left(V^{*}, h^{*}\right)\right)$. The following result states that $\mathcal{L}_{\Lambda}$ and $\mathcal{L}_{\mathrm{Cl}}$ are compatible with $\mathfrak{l}$ and $\Psi$.

Proposition 3.10 [75, Proposition 2.11] The quantization and symbol maps intertwine the actions of $V \rtimes \mathfrak{s o}\left(V^{*}, h^{*}\right)$ on $C l\left(V^{*}, h^{*}\right)$ and $\wedge V^{*}$ :

$$
\Psi\left(\mathcal{L}_{\Lambda}(\varphi)(\alpha)\right)=\mathcal{L}_{C l}(\varphi)(\Psi(\alpha)), \mathfrak{l}\left(\mathcal{L}_{C l}(\varphi)(x)\right)=\mathcal{L}_{\Lambda}(\varphi)(\mathfrak{l}(x)),
$$

for all $\varphi \in V \rtimes \mathfrak{s o}\left(V^{*}, h^{*}\right), \alpha \in \wedge V^{*}$ and $x \in C l\left(V^{*}, h^{*}\right)$.

This proposition shows that quantization is equivariant with respect to affine orthogonal transformations of $\left(V^{*}, h^{*}\right)$. In signatures $p-q \equiv_{8} 0,2$, composing $\Psi$ with the irreducible representation $\gamma: \mathrm{Cl}\left(V^{*}, h^{*}\right) \rightarrow \operatorname{End}(\Sigma)$ (which in such signatures is a unital isomorphism of algebras) gives an isomorphism of unital associative $\mathbb{R}$-algebras ${ }^{4}$ :

$$
\Psi_{\gamma} \stackrel{\text { def. }}{=} \gamma \circ \Psi:\left(\wedge V^{*}, \diamond\right) \stackrel{\sim}{\rightarrow}(\operatorname{End}(\Sigma), \circ) .
$$

Since $\Psi_{\gamma}$ is an isomorphism of algebras and $\left(\wedge V^{*}, \diamond\right)$ is generated by $V^{*}$, the identity together with the elements $\Psi_{\gamma}\left(e^{i_{1}} \wedge \cdots \wedge e^{i_{k}}\right)=\gamma\left(e^{i_{1}}\right) \circ \cdots \circ \gamma\left(e^{i_{k}}\right)$ for $1 \leq i_{1}<\cdots<i_{k} \leq d$ and $k=1, \ldots, d$ form a basis of $\operatorname{End}(\Sigma)$.

Remark 3.11 We sometimes denote the action of a polyform $\alpha \in \wedge V^{*}$ as an endomorphism on $\Sigma$ by a dot (this corresponds to Clifford multiplication through the isomorphism $\Psi_{\gamma}$ ):

$$
\alpha \cdot \xi \stackrel{\text { def. }}{=} \Psi_{\gamma}(\alpha)(\xi) \quad \forall \alpha \in \wedge V^{*} \forall \xi \in \Sigma .
$$

The trace on $\operatorname{End}(\Sigma)$ transfers to the Kähler-Atiyah algebra through the map $\Psi_{\gamma}$ (see [70]):

Definition 3.12 The Kähler-Atiyah trace is the linear functional:

$$
\mathcal{S}: \wedge V^{*} \rightarrow \mathbb{R}, \quad \alpha \mapsto \operatorname{tr}\left(\Psi_{\gamma}(\alpha)\right) .
$$

We will see in a moment that $\mathcal{S}$ does not depend on $\gamma$ or $h$. Since $\Psi_{\gamma}$ is a unital morphism of algebras, we have:

$$
\mathcal{S}(1)=\operatorname{dim}(\Sigma)=N=2^{\frac{d}{2}} \text { and } \mathcal{S}\left(\alpha_{1} \diamond \alpha_{2}\right)=\mathcal{S}\left(\alpha_{2} \diamond \alpha_{1}\right) \quad \forall \alpha_{1}, \alpha_{2} \in \wedge V^{*},
$$

where $1 \in \mathbb{R}=\wedge^{0} V^{*}$ is the unit element of the field $\mathbb{R}$ of real numbers.

Lemma 3.13 For any $0<k \leq d$, we have:

$$
\left.\mathcal{S}\right|_{\wedge^{k} V^{*}}=0 .
$$

Proof Let $\left\{e^{i}\right\}_{i=1, \ldots, d}$ be an orthonormal basis of $\left(V^{*}, h^{*}\right)$. For $i \neq j$ we have $e^{i} \diamond e^{j}=$ $-e^{j} \diamond e^{i}$ and hence $\left(e^{i}\right)^{-1} \diamond e^{j} \diamond e^{i}=-e^{j}$. Let $0<k \leq d$ and $1 \leq i_{1}<\cdots<i_{k} \leq d$. If $k$ is even, then:

$$
\mathcal{S}\left(e^{i_{1}} \diamond \cdots \diamond e^{i_{k}}\right)=\mathcal{S}\left(e^{i_{k}} \diamond e^{i_{1}} \diamond \cdots \diamond e^{i_{k-1}}\right)=(-1)^{k-1} \mathcal{S}\left(e^{i_{1}} \diamond \cdots \diamond e^{i_{k}}\right),
$$

4 This isomorphism identifies the deformation quantization $\left(\wedge V^{*}, \diamond\right)$ of the exterior algebra $\left(\wedge V^{*}, \wedge\right)$ with the operator quantization $(\operatorname{End}(\Sigma)$, o) of the latter. 
and hence $\mathcal{S}\left(e^{i_{1}} \diamond \cdots \diamond e^{i_{k}}\right)=0$. Here we used cyclicity of the Kähler-Atiyah trace and the fact that $e^{i_{k}}$ anticommutes with $e^{i_{1}}, \ldots, e^{i_{k-1}}$. If $k$ is odd, let $j \in\{1, \ldots, d\}$ be such that $j \notin\left\{i_{1}, \ldots, i_{k}\right\}$ (such a $j$ exists since $k<d$ ). We have:

$$
\mathcal{S}\left(e^{i_{1}} \diamond \cdots \diamond e^{i_{k}}\right)=\mathcal{S}\left(\left(e^{j}\right)^{-1} \diamond e^{i_{1}} \diamond \cdots \diamond e^{i_{k}} \diamond e^{j}\right)=-\mathcal{S}\left(e^{i_{1}} \diamond \cdots \diamond e^{i_{k}}\right)=0
$$

and we conclude.

Let $\alpha^{(k)} \in \wedge^{k} V^{*}$ denote the degree $k$ component of $\alpha \in \wedge V^{*}$. Lemma 3.13 implies:

Proposition 3.14 The Kähler-Atiyah trace is given by:

$$
\mathcal{S}(\alpha)=\operatorname{dim}(\Sigma) \alpha^{(0)}=2^{\frac{d}{2}} \alpha^{(0)} \quad \forall \alpha \in \wedge V^{*} .
$$

In particular, $\mathcal{S}$ does not depend on the irreducible representation $\gamma$ of $C l\left(V^{*}, h^{*}\right)$ or on $h$.

Lemma 3.15 Let $\alpha \in \wedge V^{*}$ and $\mathscr{B}$ be an admissible bilinear pairing of $(\Sigma, \gamma)$ of adjoint type $s \in\{-1,1\}$. Then the following equation holds:

$$
\Psi_{\gamma}(\alpha)^{t}=\Psi_{\gamma}\left(\alpha^{t}\right),
$$

where $\Psi_{\gamma}(\alpha)^{t}$ is the $\mathscr{B}$-adjoint of $\Psi_{\gamma}(\alpha)$ and we defined the s-transpose of $\alpha$ through:

$$
\alpha^{t} \stackrel{\text { def. }}{=}\left(\pi^{\frac{1-s}{2}} \circ \tau\right)(\alpha)
$$

Proof Follows immediately from (23) and relations (29).

\subsection{Spinor squaring maps}

Definition 3.16 Let $\Sigma=(\Sigma, \gamma, \mathscr{B})$ be a paired simple Clifford module for $\left(V^{*}, h^{*}\right)$. The signed spinor squaring maps of $\boldsymbol{\Sigma}$ are the quadratic maps:

$$
\mathcal{E}_{\Sigma}^{ \pm} \stackrel{\text { def. }}{=} \Psi_{\gamma}^{-1} \circ \mathcal{E}_{ \pm}: \Sigma \rightarrow \wedge V^{*},
$$

where $\mathcal{E}_{ \pm}: \Sigma \rightarrow \operatorname{End}(\Sigma)$ are the signed squaring maps of the paired vector space $(\Sigma, \mathscr{B})$ which were defined in Sect. 2. Given a spinor $\xi \in \Sigma$, the polyforms $\mathcal{E}_{\Sigma}^{+}(\xi)$ and $\mathcal{E}_{\Sigma}^{-}(\xi)=$ $-\mathcal{E}_{\Sigma}^{+}(\xi)$ are called the positive and negative squares of $\xi$ relative to the admissible pairing $\mathscr{B}$. A polyform $\alpha \in \wedge V^{*}$ is called a signed square of $\xi \in \Sigma$ if $\alpha=\mathcal{E}_{\Sigma}^{+}(\xi)$ or $\alpha=\mathcal{E}_{\Sigma}^{-}(\xi)$.

Consider the following subsets of $\wedge V^{*}$ :

$$
Z:=Z(\Sigma) \stackrel{\text { def. }}{=} \Psi_{\gamma}^{-1}(\mathcal{Z}(\Sigma, \mathscr{B})), \quad Z_{ \pm}:=Z_{ \pm}(\Sigma) \stackrel{\text { def. }}{=} \Psi_{\gamma}^{-1}\left(\mathcal{Z}_{ \pm}(\Sigma, \mathscr{B})\right) .
$$

Since $\Psi_{\gamma}$ is a linear isomorphism, Sect. 2 implies that $\mathcal{E}_{\Sigma}^{ \pm}$are two-to-one except at $0 \in \Sigma$ and:

$$
Z_{-}=-Z_{+}, \quad Z_{+} \cap Z_{-}=\{0\}, \quad Z=Z_{+} \cup Z_{-} .
$$

Moreover, $\mathcal{E}_{\Sigma}^{ \pm}$induce the same bijective map:

$$
\hat{\mathcal{E}}_{\Sigma}: \Sigma / \mathbb{Z}_{2} \stackrel{\sim}{\rightarrow} Z(\Sigma) / \mathbb{Z}_{2}
$$

Notice that $Z$ is a cone in $\wedge V^{*}$, which is the union of the opposite half cones $Z_{ \pm}$.

Definition 3.17 The bijection (32) is called the class spinor squaring map of the paired simple Clifford module $\Sigma=(\Sigma, \gamma, \mathscr{B})$. 
We will sometimes denote by $\alpha \xi \stackrel{\text { def. }}{=} \mathcal{E}_{\Sigma}^{+}(\xi) \in Z_{+}(\Sigma)$ the positive polyform square of $\xi \in \Sigma$.

Remark 3.18 The representation map $\gamma$ is an isomorphism when $p-q \equiv_{8} 0,2$. This does not hold in other signatures, for which the construction of spinor squaring maps is more delicate (see [70]).

The following result is a direct consequence of Proposition 3.10.

Proposition 3.19 The quadratic maps $\mathcal{E}_{\Sigma}^{ \pm}: \Sigma \rightarrow \wedge V^{*}$ are $\operatorname{Spin}_{0}\left(V^{*}, h^{*}\right)$-equivariant:

$$
\mathcal{E}_{\Sigma}^{ \pm}(u \xi)=A d_{u}\left(\mathcal{E}_{\Sigma}^{ \pm}(\xi)\right) \quad \forall u \in \operatorname{Spin}_{0}\left(V^{*}, h^{*}\right) \forall \xi \in \Sigma,
$$

where the right hand side denotes the natural action of $A d_{u} \in O\left(V^{*}, h^{*}\right)$ on $\wedge V^{*}$.

We are ready to give the algebraic characterization of spinors in terms of polyforms.

Theorem 3.20 Let $\Sigma=(\Sigma, \gamma, \mathscr{B})$ be a paired simple Clifford module for $\left(V^{*}, h^{*}\right)$ of symmetry type $\sigma$ and adjoint type $s$. Then the following statements are equivalent for a polyform $\alpha \in \wedge V^{*}$ :

(a) $\alpha$ is a signed square of some spinor $\xi \in \Sigma$, i.e. it lies in the set $Z(\Sigma)$.

(b) $\alpha$ satisfies the following relations:

$$
\alpha \diamond \alpha=\mathcal{S}(\alpha) \alpha, \quad\left(\pi^{\frac{1-s}{2}} \circ \tau\right)(\alpha)=\sigma \alpha, \quad \alpha \diamond \beta \diamond \alpha=\mathcal{S}(\alpha \diamond \beta) \alpha
$$

for a fixed polyform $\beta \in \wedge V^{*}$ which satisfies $\mathcal{S}(\alpha \diamond \beta) \neq 0$.

(c) The following relations hold:

$$
\left(\pi^{\frac{1-s}{2}} \circ \tau\right)(\alpha)=\sigma \alpha, \quad \alpha \diamond \beta \diamond \alpha=\mathcal{S}(\alpha \diamond \beta) \alpha
$$

for any polyform $\beta \in \wedge V^{*}$.

In particular, the set $Z(\Sigma)$ depends only on $\sigma, s$ and $\left(V^{*}, h^{*}\right)$.

In view of this result, we will also denote $Z(\Sigma)$ by $Z_{\sigma, s}\left(V^{*}, h^{*}\right)$.

Proof Since $\Psi: \mathrm{Cl}\left(V^{*}, h^{*}\right) \rightarrow \operatorname{End}(\Sigma)$ is a unital isomorphism algebras, $\alpha$ satisfies (34) iff:

$$
E^{t}=\sigma E, \quad E \circ A \circ E=\operatorname{tr}(E \circ A) E \quad \forall A \in \operatorname{End}(\Sigma)
$$

where $E \stackrel{\text { def. }}{=} \Psi_{\gamma}^{-1}(\alpha), A \stackrel{\text { def. }}{=} \Psi_{\gamma}^{-1}(\beta)$ and we used Lemma 3.15 and the definition and properties of the Kähler-Atiyah trace. The conclusion now follows from Proposition 2.21.

The second equation in (34) implies:

Corollary 3.21 Let $\alpha \in Z_{\sigma, s}\left(V^{*}, h^{*}\right)$. If $k \in\{1, \ldots, d\}$ satisfies:

$$
(-1)^{k \frac{1-s}{2}}(-1)^{\frac{k(k-1)}{2}}=-\sigma
$$

then $\alpha^{(k)}=0$.

Polyform $\alpha \in Z_{ \pm}(\boldsymbol{\Sigma})$ admits an explicit presentation which first appeared in $[63,66,70]$. 
Proposition 3.22 Let $\left\{e^{i}\right\}_{i=1, \ldots, d}$ be an orthonormal basis of $\left(V^{*}, h^{*}\right)$ and $\kappa \in\{-1,1\}$. Then every polyform $\alpha \in Z_{\kappa}(\mathbf{\Sigma})$ can be written as:

$$
\alpha=\frac{\kappa}{2^{\frac{d}{2}}} \sum_{k=0}^{d} \sum_{i_{1}<\cdots<i_{k}} \mathscr{B}\left(\left(\gamma\left(e^{i_{k}}\right)^{-1} \circ \cdots \circ \gamma\left(e^{i_{1}}\right)^{-1}\right)(\xi), \xi\right) e^{i_{1}} \wedge \cdots \wedge e^{i_{k}},
$$

where the spinor $\xi \in \Sigma$ is determined by $\alpha$ up to sign.

Remark 3.23 We have:

$$
\gamma\left(e^{i}\right)^{-1}=h^{*}\left(e^{i}, e^{i}\right) \gamma\left(e^{i}\right)=h\left(e_{i}, e_{i}\right) \gamma\left(e^{i}\right),
$$

where $\left\{e_{i}\right\}_{i=1, \ldots, d}$ is the contragradient orthonormal basis of $(V, h)$. For simplicity, set:

$$
\gamma \stackrel{\text { def. }}{=} \gamma\left(e^{i}\right) \text { and } \quad \gamma_{i} \stackrel{\text { def. }}{=} h\left(e_{i}, e_{i}\right) \gamma\left(e^{i}\right)
$$

so that $\left(\gamma^{i}\right)^{-1}=\gamma_{i}$. Then the degree one component in (36) reads:

$$
\alpha^{(1)}=\frac{\kappa}{2^{\frac{d}{2}}} \mathscr{B}\left(\gamma_{i}(\xi), \xi\right) e^{i}
$$

and its dual vector field $\left(\alpha^{(1)}\right)^{\sharp}=\frac{\kappa}{2^{\frac{d}{2}}} \mathscr{B}\left(\gamma_{i}(\xi), \xi\right) e_{i}$ is called the (signed) Dirac vector of $\xi$ relative to $\mathscr{B}$. For spinors on a manifold (see Sect. 4), this vector globalizes to the Dirac current.

Proof It is easy to see that the set:

$$
P \stackrel{\text { def. }}{=}\{\operatorname{Id}\} \cup\left\{\gamma^{1} \circ \cdots \circ \gamma^{i_{1}} \circ \cdots \gamma^{i_{k}} \circ \cdots \circ \gamma^{d} \mid 1 \leq i_{1}<\cdots<i_{k} \leq d, k=1, \ldots, d\right\},
$$

gives an orthogonal basis of $\operatorname{End}(\Sigma)$ with respect to the nondegenerate and symmetric bilinear pairing induced by the trace:

$$
\operatorname{End}(\Sigma) \times \operatorname{End}(\Sigma) \rightarrow \mathbb{R}, \quad\left(A_{1}, A_{2}\right) \mapsto \operatorname{tr}\left(A_{1} A_{2}\right) .
$$

In particular, the endomorphism $E \stackrel{\text { def. }}{=} \Psi_{\gamma}(\alpha) \in Z_{\kappa}(\Sigma)$ expands as:

$$
\begin{aligned}
E & =\frac{1}{2^{\frac{d}{2}}} \sum_{k=0}^{d} \sum_{i_{1}<\cdots<i_{k}} \operatorname{tr}\left(\left(\gamma^{i_{1}} \circ \cdots \circ \gamma^{i_{k}}\right)^{-1} \circ E\right) \gamma^{i_{1}} \circ \cdots \circ \gamma^{i_{k}} \\
& =\frac{\kappa}{2^{\frac{d}{2}}} \sum_{k=0}^{d} \sum_{i_{1}<\cdots<i_{k}} \mathscr{B}\left(\left(\gamma^{i_{1}} \circ \cdots \circ \gamma^{i_{k}}\right)^{-1}(\xi), \xi\right) \gamma^{i_{1}} \circ \cdots \circ \gamma^{i_{k}},
\end{aligned}
$$

where $\xi \in \Sigma$ is a spinor such that $E=\mathcal{E}_{\kappa}(\xi)$ and we noticed that $\operatorname{tr}\left(B \circ \mathcal{E}_{\kappa}(\xi)\right)=\kappa \operatorname{tr}(B(\xi) \otimes$ $\left.\xi^{*}\right)=\kappa \xi^{*}(B(\xi))=\kappa \mathscr{B}(B \xi, \xi)$ for all $B \in \operatorname{End}(\Sigma)$. The conclusion follows by applying the isomorphism algebras $\Psi_{\gamma}^{-1}:(\operatorname{End}(\Sigma), \circ) \rightarrow\left(\wedge V^{*}, \diamond\right)$ to the previous equation.

Lemma 3.24 The following identities hold for all $\alpha \in \wedge V^{*}$ :

$$
\alpha \diamond \nu=* \tau(\alpha), \quad v \diamond \alpha=*(\pi \circ \tau)(\alpha) .
$$


Proof Since multiplication by $v$ is $\mathbb{R}$-linear, it suffices to consider homogeneous elements $\alpha=e^{i_{1}} \wedge \cdots \wedge e^{i_{k}}$ with $1 \leq i_{1}<\cdots<i_{k} \leq d$, where $\left\{e^{i}\right\}_{i=1, \ldots, d}$ is an orthonormal basis of $\left(V^{*}, h^{*}\right)$. We have:

$$
\begin{aligned}
e^{i_{1}} & \wedge \cdots \wedge e^{i_{k}} \diamond v \\
= & e^{i_{1}} \diamond \cdots \diamond e^{i_{k}} \diamond e^{1} \diamond \cdots \diamond e^{d} \\
= & (-1)^{i_{1}+\cdots+i_{k}}(-1)^{k} e^{1} \diamond \cdots \diamond\left(e^{i_{1}}\right)^{2} \diamond e^{i_{1}+1} \diamond \cdots \diamond\left(e^{i_{k}}\right)^{2} \diamond e^{i_{k}+1} \diamond \cdots \diamond e^{d} \\
= & h^{*}\left(e^{i_{1}}, e^{i_{1}}\right) \cdots h^{*}\left(e^{i_{k}}, e^{i_{k}}\right)(-1)^{i_{1}+\cdots+i_{k}}(-1)^{k} e^{1} \diamond \cdots \diamond e^{i_{1}-1} \diamond \\
& e^{i_{1}+1} \cdots \diamond e^{i_{k}-1} \diamond e^{i_{k}+1} \diamond \cdots \diamond e^{d} \\
= & (-1)^{\frac{k(k-1)}{2}}(-1)^{2\left(i_{1}+\cdots+i_{k}\right)}(-1)^{2 k} *\left(e^{i_{1}} \wedge \cdots \wedge e^{i_{k}}\right)=* \tau(\alpha),
\end{aligned}
$$

which implies $\alpha \diamond v=* \tau(\alpha)$. Using the obvious relation $\alpha \diamond v=(\nu \diamond \pi)(\alpha)$, we conclude.

The following shows that the choice of admissible pairing used to construct the spinor square map is a matter of taste, see also Remark 3.4.

Proposition 3.25 Let $\xi \in \Sigma$ and denote by $\alpha_{\xi}^{ \pm} \in Z_{+}$the positive polyform squares of $\xi$ relative to the admissible pairings $\mathscr{B}_{+}$and $\mathscr{B}_{-}$of $(\Sigma, \gamma)$, which we assume to be normalized such that they are related through (27). Then the following relation holds:

$$
* \alpha_{\xi}^{+}=(-1)^{\left[\frac{q+1}{2}\right]+p(q+1)}(-1)^{d} c\left(\alpha_{\xi}^{-}\right) .
$$

where $c: \wedge V^{*} \rightarrow \wedge V^{*}$ is the linear map which acts as multiplication by $\frac{k !}{(d-k) !}$ in each degree $k$.

Proof We compute:

$$
\begin{aligned}
*\left(\alpha_{\xi}^{+}\right)^{(k)}= & \frac{1}{2^{\frac{d}{2}}} \mathscr{B}_{+}\left(\left(\gamma_{i_{k}} \circ \cdots \circ \gamma_{i_{1}}\right)(\xi), \xi\right) *\left(e^{i_{1}} \wedge \cdots \wedge e^{i_{k}}\right) \\
= & (-1)^{\left[\frac{q+1}{2}\right]+p q}(-1)^{\frac{k(k-1)}{2}} \frac{\sqrt{|h|}}{2^{\frac{d}{2}}(d-k) !} \\
& \mathscr{B}_{-}\left(\left(\gamma(v) \circ \gamma_{i_{1}} \circ \cdots \circ \gamma_{i_{k}}\right)(\xi), \xi\right) \epsilon^{i_{i_{1}} \cdots i_{k} a_{k+1} \ldots a_{d}} e_{a_{k+1}} \wedge \cdots \wedge e_{a_{d}} \\
= & (-1)^{\left[\frac{q+1}{2}\right]+p q}(-1)^{\frac{k(k-1)}{2}}(-1)^{k(d-k)} \frac{k !}{2^{\frac{d}{2}}(d-k) !} \\
& \mathscr{B}_{-}\left(\gamma(v) \gamma\left(*\left(e^{a_{k+1}} \wedge \cdots \wedge e^{d}\right)\right)(\xi), \xi\right) e_{a_{k+1}} \wedge \cdots \wedge e_{d} \\
= & (-1)^{\left[\frac{q+1}{2}\right]+p q}(-1)^{\frac{k(k-1)}{2}}(-1)^{\frac{(d-k)(d+k+1)}{2}} \frac{k !}{2^{\frac{d}{2}}(d-k) !} \\
& \mathscr{B}_{-}\left(\left(\gamma(v)^{2} \circ \gamma^{a_{k+1}} \circ \cdots \circ \gamma^{a_{d}}\right)(\xi), \xi\right) e_{a_{k+1}} \wedge \cdots \wedge e_{d} \\
= & (-1)^{\left[\frac{q+1}{2}\right]+p(q+1)}(-1)^{k} \frac{k !}{(d-k) !}\left(\alpha_{\xi}^{-}\right)^{(d-k)} \\
= & (-1)^{\left[\frac{q+1}{2}\right]+p(q+1)}(-1)^{d} \frac{k !}{(d-k) !} \pi\left(\alpha_{\xi}^{-}\right)^{(d-k)},
\end{aligned}
$$

where we used the identity $v \diamond \alpha=*(\pi \circ \tau)(\alpha)$ proved in Lemma 3.24. 


\subsection{Linear constraints}

The following result will be used in Sects. 3 and 4 .

Proposition 3.26 A spinor $\xi \in \Sigma$ lies in the kernel of an endomorphism $Q \in \operatorname{End}(\Sigma)$ iff:

$$
\hat{Q} \diamond \alpha_{\xi}=0,
$$

where $\alpha \xi \stackrel{\text { def. }}{=} \mathcal{E}_{\Sigma}^{+}(\xi)$ is the positive polyform square of $\xi$ and:

$$
\hat{Q} \stackrel{\text { def. }}{=} \Psi_{\gamma}^{-1}(Q) \in \wedge V^{*}
$$

is the dequantization of $Q$.

Remark 3.27 Taking the $s$-transpose shows that equation $\hat{Q} \diamond \alpha_{\xi}=0$ is equivalent to:

$$
\alpha_{\xi} \diamond\left(\pi^{\frac{1-s}{2}} \circ \tau\right)(\hat{Q})=0 .
$$

Proof Follows immediately from Proposition 2.28, using the fact that $\Psi_{\gamma}:\left(\wedge V^{*}, \diamond\right) \rightarrow$ $\operatorname{End}(\Sigma)$ is an isomorphism of unital associative algebras.

\subsection{Real chiral spinors}

Theorem 3.20 can be refined for chiral spinors of real type, which exist in signature $p-q \equiv_{8} 0$. In this case, the Clifford volume form $v \in \mathrm{Cl}\left(V^{*}, h^{*}\right)$ squares to 1 and lies in the center of $\mathrm{Cl}^{\mathrm{ev}}\left(V^{*}, h^{*}\right)$, giving a decomposition as a direct sum of simple associative algebras:

$$
\mathrm{Cl}^{\mathrm{ev}}\left(V^{*}, h^{*}\right)=\mathrm{Cl}_{+}^{\mathrm{ev}}\left(V^{*}, h^{*}\right) \oplus \mathrm{Cl}_{-}^{\mathrm{ev}}\left(V^{*}, h^{*}\right),
$$

where we defined:

$$
\mathrm{Cl}_{ \pm}^{\mathrm{ev}}\left(V^{*}, h^{*}\right) \stackrel{\text { def. }}{=} \frac{1}{2}(1 \pm v) \mathrm{Cl}\left(V^{*}, h^{*}\right)
$$

We decompose $\Sigma$ accordingly:

$$
\Sigma=\Sigma^{(+)} \oplus \Sigma^{(-)}, \text {where } \Sigma^{( \pm)} \stackrel{\text { def. }}{=} \frac{1}{2}(\operatorname{Id} \pm \gamma(\nu))(\Sigma) .
$$

The subspaces $\Sigma^{( \pm)} \subset \Sigma$ are preserved by the restriction of $\gamma$ to $\mathrm{Cl}^{\mathrm{ev}}\left(V^{*}, h^{*}\right)$, which therefore decomposes as a sum of two irreducible representations:

$$
\gamma^{(+)}: \mathrm{Cl}^{\mathrm{ev}}(V, h) \rightarrow \operatorname{End}\left(\Sigma^{(+)}\right) \text {and } \gamma^{(-)}: \mathrm{Cl}^{\mathrm{ev}}(V, h) \rightarrow \operatorname{End}\left(\Sigma^{(-)}\right)
$$

distinguished by the value which they take on the volume form $v \in \mathrm{Cl}^{\mathrm{ev}}\left(V^{*}, h^{*}\right)$ :

$$
\gamma^{(+)}(v)=\mathrm{Id}, \quad \gamma^{(-)}(v)=-\mathrm{Id} .
$$

A spinor $\xi \in \Sigma$ is called chiral of chirality $\mu \in\{-1,1\}$ if it belongs to $\Sigma^{(\mu)}$. Setting $\alpha_{\xi} \stackrel{\text { def. }}{=} \mathcal{E}_{\Sigma}^{+}(\xi)$, Proposition 3.26 shows that this amounts to the condition:

$$
v \diamond \alpha_{\xi}=\mu \alpha_{\xi}
$$

For any $\mu \in\{-1,1\}$ and $\kappa \in\{-1,1\}$, define:

$$
Z_{\kappa}^{(\mu)}:=Z_{\kappa}^{(\mu)}(\boldsymbol{\Sigma}) \stackrel{\text { def. }}{=} \mathcal{E}_{\boldsymbol{\Sigma}}^{\kappa}\left(\Sigma^{(\mu)}\right), \quad Z^{(\mu)}:=Z^{(\mu)}(\boldsymbol{\Sigma}) \stackrel{\text { def. }}{=} Z_{+}^{(\mu)}(\boldsymbol{\Sigma}) \cup Z_{-}^{(\mu)}(\boldsymbol{\Sigma}) .
$$


We have $Z_{-}^{(\mu)}(\boldsymbol{\Sigma})=-Z_{+}^{(\mu)}(\boldsymbol{\Sigma})$ and $Z_{+}^{(\mu)}(\boldsymbol{\Sigma}) \cap Z_{-}^{(\mu)}(\boldsymbol{\Sigma})=\{0\}$. Moreover, $\mathcal{E}_{\boldsymbol{\Sigma}}^{\kappa}$ restrict to surjections $\mathcal{E}_{\boldsymbol{\Sigma}}^{(\mu), \kappa}: \Sigma^{(\mu)} \rightarrow Z_{\kappa}^{(\mu)}(\Sigma)$ (which are two to one except at the origin). In turn, the latter induce bijections $\hat{\mathcal{E}}_{\boldsymbol{\Sigma}}^{(\mu)}: \Sigma^{(\mu)} / \mathbb{Z}_{2} \stackrel{\sim}{\rightarrow} Z^{(\mu)}(\boldsymbol{\Sigma}) / \mathbb{Z}_{2}$. Theorem 3.20, Proposition 3.26 and Lemma 3.24 give:

Corollary 3.28 Let $\mathbf{\Sigma}$ be a paired simple $C l\left(V^{*}, h^{*}\right)$-module of symmetry type $\sigma$ and adjoint type s. The following statements are equivalent for $\alpha \in \wedge V^{*}$, where $\mu \in\{-1,1\}$ is a fixed chirality type:

(a) $\alpha$ lies in the set $Z^{(\mu)}(\Sigma)$, i.e. it is a signed square of a chiral spinor of chirality $\mu$.

(b) The following conditions are satisfied:

$$
\begin{aligned}
& \left(\pi^{\frac{1-s}{2}} \circ \tau\right)(\alpha)=\sigma \alpha, \quad *(\pi \circ \tau)(\alpha)=\mu \alpha, \alpha \diamond \alpha=\mathcal{S}(\alpha) \alpha, \\
& \alpha \diamond \beta \diamond \alpha=\mathcal{S}(\alpha \diamond \beta) \alpha
\end{aligned}
$$

for a fixed polyform $\beta \in \wedge V^{*}$ which satisfies $\mathcal{S}(\alpha \diamond \beta) \neq 0$.

(c) The following conditions are satisfied:

$$
\left(\pi^{\frac{1-s}{2}} \circ \tau\right)(\alpha)=\sigma \alpha, \quad *(\pi \circ \tau)(\alpha)=\mu \alpha, \quad \alpha \diamond \beta \diamond \alpha=\mathcal{S}(\alpha \diamond \beta) \alpha
$$

for every polyform $\beta \in \wedge V^{*}$.

In this case, the real chiral spinor of chirality $\mu$ which corresponds to $\alpha$ through the either of the maps $\mathcal{E}_{\mathbf{\Sigma}}^{(\mu),+}$ or $\mathcal{E}_{\mathbf{\Sigma}}^{(\mu),-}{ }_{\text {is }}$ unique up to sign and vanishes iff $\alpha=0$.

In particular, $Z^{(\mu)}(\Sigma)$ depends only on $\sigma, s$ and $\left(V^{*}, h^{*}\right)$ and will also be denoted by $Z_{\sigma, s}^{(\mu)}\left(V^{*}, h^{*}\right)$.

Corollary 3.29 Let $\alpha \in Z_{\sigma, s}^{(+)}\left(V^{*}, h^{*}\right) \cup Z_{\sigma, s}^{(-)}\left(V^{*}, h^{*}\right)$. If $k \in\{1, \ldots, d\}$ satisfies:

$$
-(-1)^{k \frac{s-1}{2}}(-1)^{\frac{k(k-1)}{2}}=\sigma,
$$

then we have $\alpha^{(k)}=0$ and $\alpha^{(d-k)}=0$.

Proof Follows immediately from Corollary 3.21 and the second relation in (38).

\subsection{Low-dimensional examples}

Let us describe $Z$ and $Z^{(\mu)}$ for some low-dimensional cases.

\subsubsection{Signature $(2,0)$}

Let $\left(V^{*}, h^{*}\right)$ be a two-dimensional $\mathbb{R}$-vector space with a scalar product $h^{*}$. Its irreducible Clifford module $(\Sigma, \gamma)$ is two-dimensional with an admissible pairing $\mathscr{B}$ which is a scalar product. Theorem 3.20 with $\beta=1$ shows that $w \in \wedge V^{*}$ is a signed square of $\xi \in \Sigma$ iff:

$$
\alpha \diamond \alpha=2 \alpha^{(0)} \alpha, \quad \tau(\alpha)=\alpha .
$$

Writing $\alpha=\alpha^{(0)} \oplus \alpha^{(1)} \oplus \alpha^{(2)}$, the second of these relations reads:

$$
\alpha^{(0)}+\alpha^{(1)}-\alpha^{(2)}=\alpha^{(0)}+\alpha^{(1)}+\alpha^{(2)} .
$$


This gives $\alpha^{(2)}=0$, whence the first equation in (40) becomes $\left(\alpha^{(0)}\right)^{2}=h^{*}\left(\alpha^{(1)}, \alpha^{(1)}\right)$. Hence $\alpha$ is a signed square of a spinor iff:

$$
\alpha= \pm h^{*}\left(\alpha^{(1)}, \alpha^{(1)}\right)^{\frac{1}{2}} \oplus \alpha^{(1)} \text { with } \alpha^{(1)} \in V^{*} .
$$

Let $\left\{e^{i}\right\}_{i=1,2}$ be an orthonormal basis of $\left(V^{*}, h^{*}\right)$ and $\alpha=\mathcal{E}_{\Sigma}^{+}(\xi)$ for some $\xi \in \Sigma$. Then:

$$
2 \alpha=\mathscr{B}(\xi, \xi)+\mathscr{B}\left(\gamma_{i}(\xi), \xi\right) e^{i}
$$

Thus:

$$
4 h^{*}\left(\alpha^{(1)}, \alpha^{(1)}\right)=\mathscr{B}(\xi, \xi)^{2}
$$

and hence the norm of $\xi$ determines the norm of one-form $\alpha^{(1)} \in V^{*}$.

\subsubsection{Signature $(1,1)$}

Let $\left(V^{*}, h^{*}\right)$ be a two-dimensional vector space $V^{*}$ equipped with a Lorentzian metric $h^{*}$. Its irreducible Clifford module $(\Sigma, \gamma)$ is two-dimensional with a symmetric admissible bilinear pairing $\mathscr{B}$ of split signature and positive adjoint type (see Theorem 3.1). To guarantee that $\alpha \in \wedge V^{*}$ belongs to $Z$, we should in principle consider the first equation in (34) of Theorem 3.20 for all $\beta \in \wedge V^{*}$. However, $V^{*}$ is two-dimensional and Example 2.26 shows that it suffice to take $\beta=1$. Thus $\alpha$ belongs to the set $Z_{+,+}\left(V^{*}, h^{*}\right)$ iff:

$$
\alpha \diamond \alpha=2 \alpha^{(0)} \alpha, \quad \tau(\alpha)=\alpha .
$$

Writing $\alpha=\alpha^{0} \oplus \alpha^{(1)} \oplus \alpha^{(2)}$, the second condition gives $\alpha^{(2)}=0$, while the first condition becomes:

$$
\left(\alpha^{(0)}\right)^{2}=h^{*}\left(\alpha^{(1)}, \alpha^{(1)}\right) .
$$

In particular, $\alpha^{(1)}$ is space-like or null. Hence $\alpha$ is a signed square of a spinor iff:

$$
\alpha= \pm h^{*}\left(\alpha^{(1)}, \alpha^{(1)}\right)^{\frac{1}{2}}+\alpha^{(1)}
$$

for a one-form $\alpha^{(1)} \in V^{*}$. As in the Euclidean case, we have:

$$
2 \alpha=\mathscr{B}(\xi, \xi)+\mathscr{B}\left(\gamma_{i}(\xi), \xi\right) e^{i}
$$

whence:

$$
4 h^{*}\left(\alpha^{(1)}, \alpha^{(1)}\right)=\mathscr{B}(\xi, \xi)^{2} .
$$

Thus $\alpha^{(1)}$ is null iff $\mathscr{B}(\xi, \xi)=0$. In this signature the volume form squares to 1 and we have chiral spinors. Fix $\mu \in\{-1,1\}$. By Corollary 3.28, $\alpha$ lies in the set $Z_{+,+}^{(\mu)}\left(V^{*}, h^{*}\right)$ iff it has the form (42) and satisfies the supplementary condition:

$$
*(\pi \circ \tau)(\alpha)=\mu \alpha .
$$

This amounts to the following system, where $v_{h}$ is the volume form of $\left(V^{*}, h^{*}\right)$ :

$$
\pm h^{*}\left(\alpha^{(1)}, \alpha^{(1)}\right)^{\frac{1}{2}} v_{h}-* \alpha^{(1)}= \pm \mu h^{*}\left(\alpha^{(1)}, \alpha^{(1)}\right)^{\frac{1}{2}}+\mu \alpha^{(1)} .
$$

Thus $h^{*}\left(\alpha^{(1)}, \alpha^{(1)}\right)=0$ and $* \alpha^{(1)}=-\mu \alpha^{(1)}$. Hence a signed polyform square of a chiral spinor of chirality $\mu$ is a null one-form which is anti-self-dual when $\mu=+1$ and self-dual when $\mu=-1$. Notice that the nullity condition on $\alpha^{(1)}$ is equivalent with (anti-)selfduality. 


\subsubsection{Signature $(3,1)$}

This case is relevant for supergravity applications and will arise in Sects. 5 and 6. Let $\left(V^{*}, h^{*}\right)$ be a Minkowski space of "mostly plus" signature $(3,1)$. Its irreducible Clifford module $(\Sigma, \gamma)$ is four-dimensional and both admissible pairings $\mathscr{B}_{ \pm}$are skew-symmetric. We work with the admissible pairing $\mathscr{B}=\mathscr{B}$ - of negative adjoint type.

Definition 3.30 A parabolic pair of one-forms is ordered pair $(u, l) \in V^{*} \times V^{*}$ such that $u \neq 0$ and:

$$
h^{*}(u, u)=0, \quad h^{*}(l, l)=1, \quad h^{*}(u, l)=0,
$$

i.e. $u$ is nonzero and null, $l$ is spacelike of unit norm and $u$ is orthogonal to $l$. Two parabolic pairs of one forms $(u, l)$ and $\left(u^{\prime}, l^{\prime}\right)$ are called:

- weakly equivalent, if there exist $b \in \mathbb{R}^{\times}, c \in \mathbb{R}$ and $\eta \in\{-1,1\}$ such that:

$$
u^{\prime}=b u \text { and } l^{\prime}=\eta l+c u .
$$

- equivalent (and we write $\left.(u, l) \equiv\left(u^{\prime}, l^{\prime}\right)\right)$ if there exist $b \in \mathbb{R}^{\times}$and $c \in \mathbb{R}$ such that:

$$
u^{\prime}=b u \text { and } l^{\prime}=l+c u .
$$

- strongly equivalent (and we write $\left.(u, l) \sim\left(u^{\prime}, l^{\prime}\right)\right)$ if there exist $\zeta \in\{-1,1\}$ and $c \in \mathbb{R}$ such that:

$$
u^{\prime}=\zeta u \text { and } l^{\prime}=l+c u .
$$

Let $\mathcal{P}\left(V^{*}, h^{*}\right)$ denote the set of parabolic pairs of one-forms. The binary relations defined above are equivalence relations on this set; moreover, strong equivalence implies equivalence, which in turn implies weak equivalence.

Recall that a 2-plane $\Pi$ in $V^{*}$ is called parabolic (with respect to $h^{*}$ ) if the restriction $h_{\Pi}^{*}$ of $h^{*}$ to $\Pi$ has one-dimensional kernel. This happens iff $\Pi$ is tangent to the light cone of the Minkowski space $\left(V^{*}, h^{*}\right)$ along a null line. This line coincides with $K_{h}(\Pi) \stackrel{\text { def. }}{=} \operatorname{ker}\left(h_{\Pi}^{*}\right)$ and is called the null line of $\Pi$. If $\Pi \subset V^{*}$ is a parabolic 2-plane, then any element of $\Pi$ which does not belong to $\mathrm{K}_{h}(\Pi)$ is spacelike. The two connected components of the complement $\Pi \backslash \mathrm{K}_{h}(\Pi)$ are the spacelike half-planes of $\Pi$. An orientation of the null line $\mathrm{K}_{h}(\Pi)$ is called a time orientation of $\Pi$, while an orientation of the quotient line $\Pi / \mathrm{K}_{h}(\Pi)$ is called a co-orientation of $\Pi$. Notice that a co-orientation of $\Pi$ amounts to a choice $\mathcal{H}$ of one of the spacelike half-spaces of $\Pi$. A co-oriented parabolic 2-plane in $V^{*}$ is a pair $(\Pi, \mathcal{H})$, where $\Pi$ is a parabolic two-plane in $V^{*}$ and $\mathcal{H}$ is a co-orientation of $\Pi$. The set of spacelike unit norm elements of $\Pi$ has two connected components, each of which is an affine line parallel to $\mathrm{K}_{h}(\Pi)$. These two affine lines are related by the inversion of $\Pi$ with respect to the origin. Notice that a co-orientation $\mathcal{H}$ of $\Pi$ amounts to a choice $L$ of one of these two affine lines. Namely, we associate to $L$ that spacelike half-plane $\mathcal{H}_{L}$ of $\Pi$ which contains $L$. Given $u \in \mathrm{K}_{h}(\Pi) \backslash\{0\}$, a unit norm spacelike element $l \in \Pi$ is determined up to transformations of the form $l \rightarrow \zeta l+c u$, where $\zeta \in\{-1,1\}$ and $c \in \mathbb{R}$.

Remark 3.31 Parabolic 2-planes correspond to degenerate complete flags in $\left(V^{*}, h^{*}\right)$ (see Appendix A). Notice that a parabolic 2-plane $\Pi$ determines a short exact sequence of vector spaces:

$$
0 \rightarrow K \rightarrow \Pi \rightarrow N \rightarrow 0
$$


with $K=\mathrm{K}_{h}(\Pi)$ and $N=\Pi / K$ and induces a scalar product on the quotient line $N$. Conversely, giving a "parabolic" metric on a 2-plane $\Pi$ amounts to giving a short exact sequence of this form together with a scalar product on $N$. A time orientation of $\Pi$ is orientation of $K$ while a co-orientation is an orientation of $N$. Since the determinant line of $\Pi$ is given by $\operatorname{det}(\Pi)=\wedge^{2} \Pi=K \otimes L$, a time orientation and a co-orientation taken together determine an orientation of $\Pi$.

A basis of a parabolic 2-plane $\Pi \subset V^{*}$ is called parabolic if its two elements form a parabolic pair. By Sylvester's theorem, any parabolic plane $\Pi$ admits parabolic bases.

Proposition 3.32 The map $(u, l) \rightarrow \operatorname{Span}_{\mathbb{R}}(u, l)$ induces a bijection between the set of weak equivalence classes of parabolic pairs of one-forms and the set of all parabolic 2-planes in $\left(V^{*}, h^{*}\right)$.

Proof If $(u, v)$ is a parabolic pair, then $\operatorname{Span}_{\mathbb{R}}(u, v)$ is a parabolic 2-plane, which depends only on the weak equivalence class of $(u, v)$. Conversely, it is easy to see that any two parabolic bases of a parabolic 2-plane $\Pi$ in $V^{*}$ are weakly-equivalent as parabolic pairs.

Proposition 3.32 implies:

Corollary 3.33 The map $(u, l) \rightarrow\left(\operatorname{Span}_{\mathbb{R}}(u, l), \mathcal{H}_{l}\right)$ induces a bijection between the set $\mathcal{P}\left(V^{*}, h^{*}\right) / \equiv$ of equivalence classes of parabolic pairs of one-forms and the set of all cooriented parabolic 2-planes in $\left(V^{*}, h^{*}\right)$, where $\mathcal{H}_{l}$ is the unique spacelike half-plane of the parabolic 2-plane $\operatorname{Span}_{\mathbb{R}}(u, l)$ which contains the vector $l$.

Theorem 3.34 A polyform $\alpha \in \wedge V^{*}$ is a signed square of a nonzero spinor (i.e. it belongs to the set $\left.Z_{-,-}\left(V^{*}, h^{*}\right)\right)$ iff it has the form:

$$
\alpha=u+u \wedge l
$$

for a parabolic pair of one-forms $(u, l) \in \mathcal{P}\left(V^{*}, h^{*}\right)$. In this case, $u$ is uniquely determined by $\alpha$ while $l$ is determined by $\alpha$ up to transformations of the form:

$$
l \rightarrow l+c u,
$$

where $c \in \mathbb{R}$ is arbitrary. Moreover, $(u, l)$ is determined by the sign equivalence class of $\alpha$ up to strong equivalence of parabolic pairs. This gives a natural bijection between the sets $Z_{-,-}\left(V^{*}, h^{*}\right) / \mathbb{Z}_{2}$ and $\mathcal{P}\left(V^{*}, h^{*}\right) / \sim$.

Proof Let:

$$
\alpha=\sum_{k=0}^{4} \alpha^{(k)} \in \wedge V^{*} \text { where } \alpha^{(k)} \in \wedge^{k} V^{*} \forall k=0, \ldots, 4 .
$$

By Theorem 3.20, $\alpha$ lies in $Z_{-,-}\left(V^{*}, h^{*}\right)$ iff the following relations hold for $\beta=1$ and for a polyform $\beta$ such that $(\beta \diamond \alpha)^{(0)} \neq 0$ :

$$
\alpha \diamond \beta \diamond \alpha=4(\beta \diamond \alpha)^{(0)} \alpha, \quad(\pi \circ \tau)(\alpha)=-\alpha .
$$

The condition $(\pi \circ \tau)(\alpha)=-\alpha$ gives $\alpha^{(0)}=\alpha^{(3)}=\alpha^{(4)}=0$. Thus $\alpha=u+\omega$, where $u \stackrel{\text { def. }}{=} \alpha^{(1)} \in \wedge^{1} V^{*}$ and $\omega \stackrel{\text { def. }}{=} \alpha^{(2)} \in \wedge^{2} V^{*}$. For $\beta=1$, the first condition in (49) gives $(u+\omega) \diamond(u+\omega)=0$, which reduces to the following relations upon expanding the geometric product:

$$
h^{*}(u, u)=\langle\omega, \omega\rangle_{h}, \omega \wedge u=0 .
$$


Here $\langle,\rangle_{h}$ is the metric induced by $h$ on $\wedge V^{*}$. The second condition in (50) amounts to $\omega=u \wedge l$ for some $l \in V^{*}$ determined up to the transformations (48). Using this in (50) gives the condition:

$$
\left(h^{*}(l, l)-1\right) h^{*}(u, u)=h^{*}(u, l)^{2},
$$

which is invariant under the transformations (48). For $\beta=u$, the first equation in (49) amounts to $h^{*}(u, u)=0$, whence $h^{*}(u, l)=0$ by (51). It remains to show that $h^{*}(l, l)=1$. Since $u$ is non-zero and null, there exists a non-zero null one-form $v \in V^{*}$ such that $h^{*}(v, u)=1$. Then $(v \diamond v)^{(0)}=(v \diamond(u+u \wedge l))^{(0)}=h^{*}(v, u)=1$. For $\beta=v$, the first condition in (49) reduces to:

$$
(u+u \wedge l) \diamond v \diamond(u+u \wedge l)=4(u+u \wedge l) .
$$

Direct computation shows that this equation amounts to $h^{*}(l, l)=1$ and we conclude.

Remark 3.35 Given $v \in V^{*}$ with $h^{*}(v, v)=-1$, denote by $P_{v}: V^{*} \rightarrow \mathbb{R} v$ the orthogonal projection onto the line $\mathbb{R} v=\operatorname{Span}_{\mathbb{R}}(v)$. A canonical choice of $l$ is obtained by imposing the condition:

$$
P_{v}(l)=0 .
$$

Given $l \in V^{*}$ of unit norm and orthogonal to $u$, there exists a unique $c \in \mathbb{R}$ such that $P_{v}(l+$ $c u)=0$. This "choice of gauge" could be useful for spinors on time-oriented Lorentzian four-manifolds.

Corollary 3.33 and Theorem 3.34 imply the following result.

Corollary 3.36 The projective spinor squaring map $\mathbb{P} \mathcal{E}_{\Sigma}$ induces a bijection between $\mathbb{P}(\Sigma)$ and the set of all co-oriented parabolic 2-planes $(\Pi, \mathcal{H})$ in $\left(V^{*}, h^{*}\right)$. Moreover, there exist natural bijections between the following three sets:

- The set $\dot{\Sigma} / \mathbb{Z}_{2}$ of sign equivalence classes of nonzero spinors.

- The $\operatorname{set} \mathcal{P}\left(V^{*}, h^{*}\right) / \sim$ of strong equivalence classes of parabolic pairs of one-forms.

- The set of triples $(\Pi, \mathcal{H}, \hat{u})$, where $(\Pi, \mathcal{H})$ is a co-oriented parabolic 2-plane in $\left(V^{*}, h^{*}\right)$ and $\hat{u}$ is the sign equivalence class of a non-zero element $u \in K_{h}(\Pi)$.

Proof A line $\mathbb{R} \xi \in \mathbb{P}(\Sigma)$ corresponds to the line $\mathbb{R} \alpha \in \mathbb{P}\left(Z_{-,-}\left(V^{*}, h^{*}\right)\right) \subset \mathbb{P}\left(\wedge V^{*}\right)$. By Theorem 3.34, $\alpha$ determines a null one-form $u=\alpha^{(1)}$ and a line $L$ of spacelike vectors of unit norm which is parallel to the line $\operatorname{Span}_{\mathbb{R}}(u)=\mathbb{R} u$. Pick any $l \in L$ and set $\Pi \stackrel{\text { def. }}{=}$ $\operatorname{Span}(u, l)=\mathbb{R} u \oplus L$. Then $\Pi$ is a parabolic 2-plane in $V^{*}$ depending only $\mathbb{R} u$ and $L$ and we have $\mathrm{K}_{h}(\Pi)=\mathbb{R} u$. Rescaling $\alpha$ by a non-zero real number corresponds to rescaling $u=\alpha^{(1)}$ by the same. Hence $\mathbb{R} \alpha$ determines the line $\mathbb{R} u=K_{h}(\Pi)$ and relation (47) shows that $\mathbb{R} \alpha$ determines and is determined by the co-oriented parabolic 2-plane $\left(\Pi, \mathcal{H}_{L}\right)$. This proves the first statement.

Now recall that the sign-equivalence class of a non-zero spinor $\xi$ determines and is determined by the sign equivalence class of its square polyform $\alpha$ through the map $\hat{\mathcal{E}}_{\boldsymbol{\Sigma}}$. By (47), the sign change $\alpha \rightarrow-\alpha$ corresponds to $u \rightarrow-u$ and $l \rightarrow l$. Thus the sign equivalence class of $u$ is uniquely determined by that of $\alpha$ and hence by that of $\xi$. This establishes the bijection between the three sets in the second statement.

Remark 3.37 A parabolic pair $(u, l)$ and a polyform square $\alpha$ are recovered from the triplet $(\Pi, \mathcal{H}, \hat{u})$ by taking $u$ to be any representative of the sign equivalence class $\hat{u}$ and $l$ to be any vector lying on the unit norm affine line $L$ contained in $\mathcal{H}$ and setting $\alpha=u+u \wedge l$. 
Let us count the degrees of freedom encoded in $\alpha=u+u \wedge l$. Apriori, the null one-form $u$ has three degrees of freedom while the space-like one-form $l$ has four, which are reduced to two by the requirement that $l$ has unit norm and is orthogonal to $u$. Since $l$ is defined only up to $l \mapsto l+c u(c \in \mathbb{R})$, its degrees of freedom further reduce from two to one. This gives a total of four degrees of freedom, matching those of a real spinor in four-dimensional Lorentzian signature.

\subsubsection{Signature $(2,2)$}

Let $\left(V^{*}, h^{*}\right)$ be four-dimensional with metric $h^{*}$ of split signature. Its Clifford module $(\Sigma, \gamma)$ is four-dimensional and has a skew-symmetric admissible pairing $\mathscr{B}$ of positive adjoint type (see Theorem 3.1). This dimension and signature admits chiral spinors. Let:

$$
\alpha=\sum_{k=0}^{4} \alpha^{(k)} \in \wedge V^{*} \text { with } \alpha^{(k)} \in \wedge^{k} V^{*} \quad \forall k=1, \ldots 4 .
$$

Fixing an orthonormal basis $\left\{e^{a}\right\}_{a=1, \ldots, 4}$ of $\left(V^{*}, h^{*}\right)$ with $e^{1}, e^{2}$ timelike, define timelike and spacelike volume forms through $v_{-}=e^{1} \wedge e^{2}$ and $v_{+}=e^{3} \wedge e^{4}$. By Corollary 3.28, we have $\alpha \in Z_{-,+}^{(\mu)}\left(V^{*}, h^{*}\right)$ iff:

$$
\alpha \diamond \alpha=0, \quad \tau(\alpha)=-\alpha, \quad * \pi(\tau(\alpha))=\mu \alpha, \quad \alpha \diamond \beta \diamond \alpha=4(\beta \diamond \alpha)^{(0)} \alpha
$$

for a polyform $\beta \in \wedge V^{*}$ such that $(\beta \diamond \alpha)^{(0)} \neq 0$. Here we used skew-symmetry of $\mathscr{B}$, which implies $\alpha^{(0)}=0$. The condition $\tau(\alpha)=-\alpha$ amounts to:

$$
\alpha^{(0)}=\alpha^{(1)}=\alpha^{(4)}=0
$$

whereas the condition $* \pi(\tau(\alpha))=\mu \alpha$ is equivalent with:

$$
* \alpha^{(2)}=-\mu \alpha^{(2)}, \alpha^{(3)}=0 .
$$

Thus it suffices to consider $\alpha=\omega$, where $\omega$ is selfdual if $\mu=-1$ and anti-selfdual if $\mu=1$. In signature $(2,2)$, the Hodge star operator squares to the identity and yields a decomposition:

$$
\wedge^{2} V^{*}=\wedge_{+}^{2} V^{*} \oplus \wedge_{-}^{2} V^{*},
$$

into self-dual and anti-selfdual two-forms. This corresponds to the decomposition $\mathfrak{s o}(2,2)=$ $\mathfrak{s l}(2) \oplus \mathfrak{s l}(2)$ of the Lie algebra $\mathfrak{s o}(2,2)=\wedge^{2} V^{*}$. Expanding the geometric product shows that the first equation in (52) reduces to the following condition for a selfdual or anti-selfdual two-form $\alpha=\omega$ :

$$
\langle\omega, \omega\rangle_{h}=0 .
$$

For simplicity of exposition we set $\mu=-1$ in what follows, in which case $\omega$ is self-dual (analogous results hold for $\mu=1$ ). Consider the basis $\left\{u_{a}\right\}_{a=1,2,3}$ of $\wedge_{+}^{2} V^{*}$ given by:

$$
u_{1} \stackrel{\text { def. }}{=} e^{1} \wedge e^{2}+e^{3} \wedge e^{4}, \quad u_{2} \stackrel{\text { def. }}{=} e^{1} \wedge e^{3}+e^{2} \wedge e^{4}, \quad u_{3} \stackrel{\text { def. }}{=} e^{1} \wedge e^{4}-e^{2} \wedge e^{3},
$$

and expand:

$$
\omega=\sum k^{a} u_{a}
$$


We have:

$$
\begin{aligned}
& v_{-} \diamond u_{1}=u_{1} \diamond v_{-}=-1+v_{h}, \quad v_{-} \diamond u_{2}=-u_{2} \diamond v_{-}=-u_{3}, \\
& v_{-} \diamond u_{3}=-u_{3} \diamond v_{-}=u_{2},
\end{aligned}
$$

which gives:

$$
\left(\nu_{-} \diamond \omega\right)^{(0)}=-k^{1} .
$$

Furthermore, we compute:

$$
\begin{aligned}
& u_{1} \diamond u_{3}=-u_{3} \diamond u_{1}=2 u_{2}, \quad u_{1} \diamond u_{2}=-u_{2} \diamond u_{1}=-2 u_{3}, \\
& u_{2} \diamond u_{3}=-u_{3} \diamond u_{2}=2 u_{1}, \\
& u_{1} \diamond u_{1}=-u_{2} \diamond u_{2}=-u_{3} \diamond u_{2}=-2+2 v_{h} .
\end{aligned}
$$

These products realize the Lie algebra $\mathfrak{s l}(2, \mathbb{R})$ upon defining a Lie bracket by the commutator:

$$
\begin{aligned}
& {\left[u_{1}, u_{2}\right]=u_{1} \diamond u_{2}-u_{2} \diamond u_{1}=-4 u_{3},\left[u_{1}, u_{3}\right]=u_{1} \diamond u_{3}-u_{3} \diamond u_{1}=4 u_{2},} \\
& {\left[u_{2}, u_{3}\right]=u_{2} \diamond u_{3}-u_{3} \diamond u_{2}=4 u_{1} .}
\end{aligned}
$$

Since $\wedge_{+}^{2} V^{*}=\mathfrak{s l}(2, \mathbb{R})$, the Killing form $\mathfrak{B}$ of $\mathfrak{s l}(2, \mathbb{R})$ gives a symmetric non-degenerate pairing of signature $(1,2)$ on $\wedge_{+}^{2} V^{*}$, which can be rescaled to coincide with that induced induced by $h$. Then:

$$
\mathfrak{B}(\omega, \omega)=\langle\omega, \omega\rangle_{h}^{2}=2\left[\left(k^{1}\right)^{2}-\left(k^{2}\right)^{2}-\left(k^{3}\right)^{2}\right] \quad \forall \omega \in \wedge_{+}^{2} V^{*} .
$$

Proposition 3.38 A polyform $\alpha \in \wedge V^{*}$ is a signed square of a real chiral spinor $\xi \in \Sigma^{(-)}$ of negative chirality iff $\alpha$ is a self-dual two-form of zero norm.

Proof It suffices to consider the case $\alpha \neq 0$. By the discussion above, a non-zero polyform $\alpha \neq 0$ belongs to the set $Z_{-,+}^{(-)}\left(V^{*}, h^{*}\right)$ only if $\alpha=\omega$ is self-dual and of zero norm (which is equivalent to the first three equations in (52)). Once these conditions are satisfied, the only equation that remains to be solved is the fourth equation in (52). To solve it, we take $\beta=v_{-}$. Since $\left(\nu_{-} \diamond \omega\right)^{(0)}=-4 k^{1}$ (as remarked above), we conclude that $\left(\nu_{-} \diamond \omega\right)^{(0)} \neq 0$ iff $\omega \neq 0$, whence taking $\beta=v_{-}$is a valid choice. A computation shows that this equation is automatically satisfied and thus we conclude.

Remark 3.39 Section 3.6.2 together with Proposition 3.38 show that the square of a chiral spinor in signatures $(1,1)$ and $(2,2)$ is given by an (anti-)self-dual form of zero norm in middle degree. The reader can verify, through a computation similar to the one presented in this subsection, that the same statement holds in signature $(3,3)$. It is tempting to conjecture that the square of a chiral spinor in general split signature $(p, p)$ corresponds to an (anti)self-dual $p$-form of zero norm, the latter condition being automatically implied when $p$ is odd. Verifying this conjecture would be useful in the study of manifolds of split signature which admit parallel chiral spinors [25].

\section{Constrained generalized Killing spinors of real type}

To study constrained generalized Killing spinors of real type, we will extend the theory of Sect. 3 to bundles of real irreducible Clifford modules equipped with an arbitrary connection. Throughout this section, let $(M, g)$ denote a connected pseudo-Riemannian manifold of 
signature $(p, q)$ and even dimension $d=p+q \geq 2$, where $p-q \equiv_{8} 0,2$. Since $M$ is connected, the pseudo-Euclidean vector bundle $(T M, g)$ is modeled on a fixed quadratic vector space denoted by $(V, h)$. For any point $m \in M$, we thus have an isomorphism of quadratic spaces $\left(T_{m} M, g_{m}\right) \simeq(V, h)$. Accordingly, the cotangent bundle $T^{*} M$ (endowed with the dual metric $\left.g^{*}\right)$ is modeled on the dual quadratic space $\left(V^{*}, h^{*}\right)$. We denote by $\mathrm{Cl}(M, g)$ the bundle of real Clifford algebras of the cotangent bundle $\left(T^{*} M, g^{*}\right)$, which is modeled on the real Clifford algebra $\mathrm{Cl}\left(V^{*}, h^{*}\right)$. Let $\pi$ and $\tau$ be the canonical automorphism and anti-automorphism of the Clifford bundle, given by fiberwise extension of the corresponding objects defined in Sect. 3 and set $\hat{\pi}=\pi \circ \tau$. We denote by $(\Lambda(M), \diamond)$ the exterior bundle $\Lambda(M)=\oplus_{j=0}^{d} \wedge^{j} T^{*} M$, equipped with the pointwise extension $\diamond$ of the geometric product of Sect. 3 (which depends on the metric $g$ ). This bundle of unital associative algebras is called the Kähler-Atiyah bundle of $(M, g)$ (see [66,70]). The map $\Psi$ of Sect. 3 extends to a unital isomorphism of bundles of algebras:

$$
\Psi:(\Lambda(M), \diamond) \stackrel{\sim}{\rightarrow} \mathrm{Cl}(M, g),
$$

which allows us to view the Kähler-Atiyah bundle as a model for the Clifford bundle. We again denote by $\pi, \tau$ and $\hat{\pi}=\pi \circ \tau$ the (anti-)automorphisms of the Kähler-Atiyah bundle obtained by transporting the corresponding objects from the Clifford bundle through $\Psi$. The Kähler-Atiyah trace of Sect. 3 extends to a morphism of vector bundles:

$$
\mathcal{S}: \Lambda(M) \rightarrow \mathbb{R}_{M}
$$

whose induced map on smooth sections satisfies:

$$
\mathcal{S}\left(1_{M}\right)=N=2^{\frac{d}{2}} 1_{M} \text { and } \mathcal{S}\left(\omega_{1} \diamond \omega_{2}\right)=\mathcal{S}\left(\omega_{2} \diamond \omega_{1}\right) \quad \forall \omega_{1}, \omega_{2} \in \Omega^{*}(M),
$$

where $1_{M} \in \Gamma\left(\mathbb{R}_{M}\right)=\Omega^{0}(M)$ is the unit function defined on $M$. By Proposition 3.14, we have:

$$
\mathcal{S}(\omega)=2^{\frac{d}{2}} \omega^{(0)} \quad \forall \omega \in \Omega(M) .
$$

In particular, $\mathcal{S}$ does not depend on the metric $g$. The following encodes a well-know property of the Clifford bundle, which also follows from the definition of $\diamond$ (cf. [66,70]).

Proposition 4.1 The canonical extension to $\Lambda(M)$ of the Levi-Civita connection $\nabla^{g}$ of $(M, g)$ to $\Lambda(M)$ (which we again denote by $\nabla^{g}$ ) acts by derivations of the geometric product:

$$
\nabla^{g}(\alpha \diamond \beta)=\left(\nabla^{g} \alpha\right) \diamond \beta+\alpha \diamond\left(\nabla^{g} \beta\right) \quad \forall \alpha, \beta \in \Omega(M) .
$$

\subsection{Bundles of real simple Clifford modules}

Definition 4.2 A bundle of (real) Clifford modules on $(M, g)$ is a pair $(S, \Gamma)$, where $S$ is a real vector bundle on $M$ and $\Gamma: \operatorname{Cl}(M, g) \rightarrow \operatorname{End}(S)$ is a unital morphism of bundles of algebras (which we call the structure map).

Since $M$ is connected, any bundle of Clifford modules $(S, \Gamma)$ on $(M, g)$ is modeled on a Clifford representation $\gamma: \mathrm{Cl}\left(V^{*}, h^{*}\right) \rightarrow \operatorname{End}(\Sigma)$ (called its model representation), where $\Sigma$ is a vector space isomorphic to the fiber of $S$. For every point $m \in M$, the unital morphism of associative algebras $\Gamma_{m}: \operatorname{Cl}\left(T_{m}^{*} M, g_{m}^{*}\right) \rightarrow\left(\operatorname{End}\left(S_{m}\right)\right.$, o $)$ identifies with the representation morphism $\gamma$ upon composing appropriately with the unital algebra isomorphisms $\mathrm{Cl}\left(T_{m}^{*}, g_{m}^{*}\right) \simeq \mathrm{Cl}\left(V^{*}, h^{*}\right)$ and $\operatorname{End}\left(S_{m}\right) \simeq \operatorname{End}(\Sigma)$ (the latter of which is induced by the linear isomorphism $S_{m} \simeq \Sigma$ ). 
Definition 4.3 We say that $(S, \Gamma)$ is a bundle of simple real Clifford modules (or a real spinor bundle) if its model representation $\gamma$ is irreducible. In this case, a global section $\epsilon \in \Gamma(S)$ is called $^{5}$ a spinor on $(M, g)$.

In the signatures $p-q \equiv_{8} 0,2$ considered in this paper, a simple bundle of Clifford modules satisfies rk $S=\operatorname{dim} V=2^{\frac{d}{2}}$, where $d$ is the dimension of $M$. Reference [69] proves that $(M, g)$ admits a bundle of simple real Clifford modules iff it admits a real Lipschitz structure of type $\gamma$. In signatures $p-q \equiv_{8} 0,2$, the latter corresponds to an adjointequivariant (a.k.a. "untwisted") $\operatorname{Pin}\left(V^{*}, h^{*}\right)$-structure $\mathfrak{Q}$ on $(M, g)$ and $(S, \Gamma)$ is isomorphic (as a unital bundle of algebras) with the bundle of real Clifford modules associated to $\mathfrak{Q}$ through the natural representation of $\operatorname{Pin}\left(V^{*}, h^{*}\right)$ in $\Sigma$. The obstructions to existence of such structures were given in [69]; when $p-q \equiv_{8} 0,2$, they are a slight modification of those given in [62] for ordinary (twisted adjoint-equivariant) $\operatorname{Pin}\left(V^{*}, h^{*}\right)$-structures.

Proposition 4.4 Let $(S, \Gamma)$ be a bundle of real Clifford modules on $(M, g), L$ a real line bundle on $M$ and set $S_{L} \stackrel{\text { def. }}{=} S \otimes L$. Then there exists a natural unital morphism of bundles of algebras $\Gamma_{L}: C l(M, g) \rightarrow \operatorname{End}(S \otimes L)$. Hence the modification $\left(S_{L}, \Gamma_{L}\right)$ of $(S, \Gamma)$ by $L$ is a bundle of Clifford modules, which is a real spinor bundle iff $(S, L)$ is. In particular, the real Picard group Pic $(M)$ acts naturally on the set of isomorphism classes of bundles of real Clifford modules defined over $(M, g)$.

Proof There exists a unique trivialization $\psi_{L}: \operatorname{End}(L) \simeq \mathbb{R}_{M}$ of the line bundle $\operatorname{End}(L)$ which is a unital isomorphism of bundles of $\mathbb{R}$-algebras-namely that trivialization which sends the identity endomorphism of $L$ into the unit section of $\mathbb{R}_{M}$ (which is the constant function equal to 1 defined on $M$ ). This induces a unital isomorphism of bundles of algebras $\varphi_{L}: \operatorname{End}(S \otimes L) \stackrel{\sim}{\rightarrow} \operatorname{End}(S)$ given by composing the natural isomorphism of bundles of $\mathbb{R}$-algebras $\operatorname{End}(S \otimes L) \stackrel{\sim}{\rightarrow} \operatorname{End}(S) \otimes \operatorname{End}(L)$ with $\operatorname{Id}_{E n d(S)} \otimes \psi_{L}$. The conclusion follows by setting $\Gamma_{L} \stackrel{\text { def. }}{=} \varphi_{L}^{-1} \circ \Gamma$.

The map $\Psi_{\gamma}$ of Sect. 3 extends to a unital isomorphism of bundles of algebras:

$$
\Psi_{\Gamma} \stackrel{\text { def. }}{=} \Gamma \circ \Psi:(\Lambda(M), \diamond) \stackrel{\sim}{\rightarrow}(\operatorname{End}(S), \circ),
$$

which allows us to identify bundles $(S, \Gamma)$ of modules over $\mathrm{Cl}\left(T^{*} M, g^{*}\right)$ with bundles of modules $\left(S, \Psi_{\Gamma}\right)$ over the Kähler-Atiyah algebra. We denote by a dot the external multiplication $^{6}$ of $\left(S, \Psi_{\Gamma}\right)$, whose action on global sections is:

$$
\alpha \cdot \epsilon \stackrel{\text { def. }}{=} \Psi_{\Gamma}(\alpha)(\epsilon) \quad \forall \alpha \in \Omega(M) \stackrel{\text { def. }}{=} \Gamma(\Lambda(M)) \forall \epsilon \in \Gamma(S) .
$$

Let $\operatorname{tr}: \operatorname{End}(S) \rightarrow \mathbb{R}_{M}$ be the fiberwise trace morphism, whose map induced on sections we denote by the same symbol. The results of Sect. 3 imply:

Proposition 4.5 Let $(S, \Gamma)$ be a real spinor bundle. Then:

$$
\mathcal{S}(\omega)=\operatorname{tr}\left(\Psi_{\Gamma}(\omega)\right) \quad \forall \omega \in \Omega(M) .
$$

\footnotetext{
5 Since $S$ need not be associated to a spin structure on $(M, g)$, this generalizes the traditional notion of spinor. In signatures $p-q \equiv_{8} 0,2, S$ is associated to an untwisted Pin structure (see [69]) so its sections could also be called "pinors".

6 Through the isomorphisms explained above, this corresponds to Clifford multiplication on the vector bundle $S$, whose existence amounts to existence of the corresponding real Lispchitz structure on $(M, g)$ by the results of [69].
} 
Definition 4.6 Let $(S, \Gamma)$ be a real spinor bundle on $(M, g)$ and $U$ be any vector bundle on $M$. The symbol of a section $W \in \Gamma(E n d(S) \otimes U)$ is the section $\hat{W} \in \Gamma(\Lambda(M) \otimes U)$ defined through:

$$
\hat{W} \stackrel{\text { def. }}{=}\left(\Psi_{\Gamma} \otimes \operatorname{Id}_{U}\right)^{-1}(T) \in \Gamma\left(\wedge T^{*} M \otimes U\right),
$$

where $\operatorname{Id}_{U}$ is the identity endomorphism of $U$.

Remark 4.7 In particular, the symbol of an endomorphism $\mathcal{Q} \in \Gamma(\operatorname{End}(S))$ is a polyform $\hat{\mathcal{Q}} \in \Omega(M)$, while the symbol of an $\operatorname{End}(S)$-valued one-form $\mathcal{A} \in \Gamma\left(T^{*} M \otimes \operatorname{End}(S)\right)$ is an element $\hat{\mathcal{A}} \in \Gamma\left(M, T^{*} M \otimes \wedge T^{*} M\right)=\Omega^{1}(M, \Lambda(M))=\Omega^{*}\left(M, T^{*} M\right)$, which can be viewed as a $T^{*} M$-valued polyform or as a $\Lambda(M)$-valued 1-form.

\subsection{Paired spinor bundles}

Definition 4.8 Let $(S, \Gamma)$ be a real spinor bundle on $(M, g)$. A fiberwise-bilinear pairing $\mathscr{B}$ on $S$ is called admissible if $\mathscr{B}_{m}: S_{m} \times S_{m} \rightarrow \mathbb{R}$ is an admissible pairing on the simple Clifford module $\left(S_{m}, \Gamma_{m}\right)$ for all $m \in M$. A (real) paired spinor bundle on $(M, g)$ is a triplet $\mathbf{S}=(S, \Gamma, \mathscr{B})$, where $(S, \Gamma)$ is a real spinor bundle on $(M, g)$ and $\mathscr{B}$ is an admissible pairing on $S$.

Since $M$ is connected, the symmetry and adjoint type $\sigma, s \in\{-1,1\}$ of the admissible pairings $\mathscr{B}_{m}$ (which are non-degenerate by definition) are constant on $M$; they are called the symmetry type and adjoint type of $\mathscr{B}$ and of $(S, \Gamma, \mathscr{B})$. An admissible pairing on $(S, \Gamma)$ can be viewed as a morphism of vector bundles $\mathscr{B}: S \otimes S \rightarrow \mathbb{R}_{M}$, where $\mathbb{R}_{M}$ is the trivial real line bundle on $M$. Since $M$ is paracompact, the defining algebraic properties of an admissible pairing can be formulated equivalently as follows using global sections (see [70]), when viewing $(S, \Gamma)$ as a bundle $\left(S, \Psi_{\Gamma}\right)$ of modules over the Kähler-Atiyah algebra of $(M, g)$ :

1. $\mathscr{B}\left(\xi_{1}, \xi_{2}\right)=\sigma \mathscr{B}\left(\xi_{2}, \xi_{2}\right) \quad \forall \xi_{1}, \xi_{2} \in \Gamma(S)$

2. $\mathscr{B}\left(\Psi_{\Gamma}(\omega) \xi_{1}, \xi_{2}\right)=\mathscr{B}\left(\xi_{1}, \Psi_{\Gamma}\left(\left(\pi^{\frac{1-s}{2}} \circ \tau\right)(\omega)\right)\left(\xi_{2}\right)\right) \forall \omega \in \Omega(M) \quad \forall \xi_{1}, \xi_{2} \in \Gamma(S)$.

Definition 4.9 We say that $(M, g)$ is strongly spin if it admits a $\operatorname{Spin}_{0}\left(V^{*}, h^{*}\right)$-structurewhich we call a strong spin structure. In this case, a real spinor bundle $(S, \Gamma)$ on $(M, g)$ is called strong if it associated to a strong spin structure.

When $(M, g)$ is strongly spin, then it is strongly orientable in the sense that its orthonormal coframe bundle admits a reduction to an $\mathrm{SO}_{0}\left(V^{*}, h^{*}\right)$-bundle.

Remark 4.10 When $p q=0$, the special orthogonal and spin groups are connected while the pin group has two connected components. In this case, orientability and strong orientability are equivalent, as are the properties of being spin and strongly spin. When $p q \neq 0$, the groups $\operatorname{SO}\left(V^{*}, h^{*}\right)$ and $\operatorname{Spin}\left(V^{*}, h^{*}\right)$ have two connected components, while $\operatorname{Pin}\left(V^{*}, h^{*}\right)$ has four and we have $\operatorname{Pin}\left(V^{*}, h^{*}\right) / \operatorname{Spin}_{0}\left(V^{*}, h^{*}\right) \simeq \mathbb{Z}_{2} \times \mathbb{Z}_{2}$. In this case, $(M, g)$ is strongly orientable iff it is orientable and in addition the principal $\mathbb{Z}_{2}$-bundle associated to its bundle of oriented coframes through the group morphism $\mathrm{SO}\left(V^{*}, h^{*}\right) \rightarrow \mathrm{SO}\left(V^{*}, h^{*}\right) / \mathrm{SO}_{0}\left(V^{*}, h^{*}\right)$ is trivial, while an untwisted $\operatorname{Pin}\left(V^{*}, h^{*}\right)$-structure $\mathfrak{Q}$ reduces to a $\operatorname{Spin}_{0}\left(V^{*}, h^{*}\right)$-structure iff the principal $\mathbb{Z}_{2} \times \mathbb{Z}_{2}$-bundle associated to $\mathfrak{Q}$ through the group morphism $\operatorname{Pin}\left(V^{*}, h^{*}\right) \rightarrow$ $\operatorname{Pin}\left(V^{*}, h^{*}\right) / \operatorname{Spin}_{0}\left(V^{*}, h^{*}\right)$ is trivial. When $(M, g)$ is strongly spin, the short exact sequence:

$$
1 \rightarrow \mathbb{Z}_{2} \hookrightarrow \operatorname{Spin}_{0}\left(V^{*}, h^{*}\right) \rightarrow \operatorname{SO}_{0}\left(V^{*}, h^{*}\right) \rightarrow 1
$$


induces a sequence in Cech cohomology which implies that $\operatorname{Spin}_{0}\left(V^{*}, h^{*}\right)$-structures form a torsor over $H^{1}\left(M, \mathbb{Z}_{2}\right)$. A particularly simple case arises when $H^{1}\left(M, \mathbb{Z}_{2}\right)=0$ (for example, when $M$ is simply-connected). In this situation, $M$ is strongly orientable and any untwisted $\operatorname{Pin}\left(V^{*}, h^{*}\right)$-structure on $(M, g)$ reduces to a $\operatorname{Spin}_{0}\left(V^{*}, h^{*}\right)$-structure since $H^{1}\left(M, \mathbb{Z}_{2} \times\right.$ $\left.\mathbb{Z}_{2}\right)=H^{1}\left(M, \mathbb{Z}_{2} \oplus \mathbb{Z}_{2}\right)=0$. Similarly, any $\operatorname{Spin}\left(V^{*}, h^{*}\right)$-structure on $(M, g)$ reduces to a $\operatorname{Spin}_{0}\left(V^{*}, h^{*}\right)$-structure. Up to isomorphism, in this special case there exists at most one $\operatorname{Spin}\left(V^{*}, h^{*}\right)$-structure, one $\operatorname{Spin}_{0}\left(V^{*}, h^{*}\right)$-structure and one real spinor bundle on $(M, g)$, which is automatically strong.

The following gives sufficient conditions for existence of admissible pairings on real spinor bundles:

Proposition 4.11 Suppose that $(M, g)$ is strongly spin and Let $(S, \Gamma)$ be a strong real spinor bundle on $(M, g)$. Then every admissible pairing on $(\Sigma, \gamma)$ extends to an admissible pairing $\mathscr{B}$ on $(S, \Gamma)$. Moreover, the Levi-Civita connection $\nabla^{g}$ of $(M, g)$ lifts to a unique connection on $S$ (denoted $\nabla^{S}$ and called the spinorial connection of $S$ ), which acts by module derivations:

$$
\nabla_{X}^{S}(\alpha \cdot \epsilon)=\left(\nabla_{X}^{g} \alpha\right) \cdot \epsilon+\alpha \cdot\left(\nabla_{X}^{S} \epsilon\right) \quad \forall \alpha \in \Omega(M) \forall \epsilon \in \Gamma(S) \forall X \in \mathfrak{X}(M)
$$

and is compatible with $\mathscr{B}$ :

$$
X\left[\mathscr{B}\left(\epsilon_{1}, \epsilon_{2}\right)\right]=\mathscr{B}\left(\nabla_{X}^{S} \epsilon_{1}, \epsilon_{2}\right)+\mathscr{B}\left(\epsilon_{1}, \nabla_{X}^{S} \epsilon_{2}\right) \quad \forall \epsilon_{1}, \epsilon_{2} \in \Gamma(S) \forall X \in \mathfrak{X}(M) .
$$

Proof The first statement follows from the associated bundle construction since admissible pairings are $\operatorname{Spin}_{0}\left(V^{*}, h^{*}\right)$-invariant by Proposition 3.7. The second and third statements are standard (see [30, Chapter 3]). The last statement follows since the holonomy of $\nabla^{S}$ is contained in $\operatorname{Spin}_{0}\left(V^{*}, h^{*}\right)$, whose action on $\Sigma$ preserves $\mathscr{B}$.

With the assumptions of the proposition, the spinorial connection induces a linear connection (denoted $D^{S}$ ) on the bundle of endomorphisms $E n d(S)=S^{*} \otimes S$. By definition, we have:

$$
\left(D_{X}^{S} A\right)(\epsilon)=\nabla_{X}^{S}[A(\epsilon)]-A\left(\nabla_{X}^{S} \epsilon\right) \quad \forall A \in \Gamma(\operatorname{End}(S)) \quad \forall \epsilon \in \Gamma(S) \forall X \in \mathfrak{X}(M) .
$$

Proposition 4.12 Suppose that $(M, g)$ is strongly spin and let $(\Sigma, \Gamma)$ be a strong real spinor bundle over $(M, g)$. Then $D^{S}: \Gamma(E n d(S)) \rightarrow \Gamma\left(T^{*} M \otimes E n d(S)\right)$ acts by derivations:

$$
D_{X}^{S}\left(A_{1} \circ A_{2}\right)=D_{X}^{S}\left(A_{1}\right) \circ A_{2}+A_{1} \circ D_{X}^{S}\left(A_{2}\right) \quad \forall A_{1}, A_{2} \in \Gamma(\operatorname{End}(S)) \forall X \in \mathfrak{X}(M) .
$$

Moreover, $\Psi_{\Gamma}$ induces a unital isomorphism of algebras $(\Omega(M), \diamond) \simeq(\Gamma(\operatorname{End}(S)), \circ)$ which is compatible with $\nabla^{g}$ and $D^{S}$ :

$$
D_{X}^{S}\left(\Psi_{\Gamma}(\alpha)\right)=\Psi_{\Gamma}\left(\nabla_{X}^{g} \alpha\right) \quad \forall \alpha \in \Omega(M) \forall X \in \mathfrak{X}(M) .
$$

Proof That $D^{S}$ acts by algebra derivations of $\Gamma(\operatorname{End}(S))$ is standard. Proposition 4.11 gives:

$$
\left(D_{X}^{S} A\right)(\epsilon)=\nabla_{X}^{S} A(\epsilon)-A\left(\nabla_{X}^{S} \epsilon\right)=\nabla_{X}^{S}\left(\Psi_{\Gamma}(\alpha)(\epsilon)\right)-\Psi_{\Gamma}(\alpha)\left(\nabla_{X}^{S} \epsilon\right)=\Psi_{\Gamma}\left(\nabla_{X}^{g} \alpha\right)(\epsilon)
$$

for all $A \in \Gamma(\operatorname{End}(S)), \epsilon \in \Gamma(S)$ and $X \in \mathfrak{X}(M)$, where $\alpha \stackrel{\text { def. }}{=} \Psi_{\Gamma}^{-1}(A) \in \Omega(M)$.

Definition 4.13 Suppose that $(M, g)$ is strongly spin and let $(S, \Gamma)$ be a strong real spinor bundle over $(M, g)$. Given a connection $\mathcal{D}: \Gamma(S) \rightarrow \Omega^{1}(M, S)$ on $S$, its dequantization is the connection $\hat{\mathcal{D}}: \Gamma(\Lambda(M)) \rightarrow \Omega^{1}(M, \Lambda(M))$ defined on $\Lambda(M)$ through:

$$
\hat{\mathcal{D}}_{X} \stackrel{\text { def. }}{=} \Psi_{\Gamma}^{-1} \circ \mathcal{D}_{X} \circ \Psi_{\Gamma} \quad \forall X \in \mathfrak{X}(M) .
$$


Remark 4.14 Writing $\mathcal{D}=\nabla^{S}-\mathcal{A}$ with $\mathcal{A} \in \Omega^{1}(\operatorname{End}(S))$, we have:

$$
\hat{\mathcal{D}}=\nabla^{g}-\hat{\mathcal{A}},
$$

where $\hat{\mathcal{A}} \in \Omega^{1}(M, \Lambda(M))$ is the symbol of $\mathcal{A}$, which we shall also call the symbol of $\mathcal{D}$.

\subsection{Constrained generalized Killing spinors}

Definition 4.15 Let $(S, \Gamma)$ be a real spinor bundle on $(M, g)$ and $\mathcal{D}$ be an arbitrary connection on $S$. A section $\epsilon \in \Gamma(S)$ is called generalized Killing spinor with respect to $\mathcal{D}$ if:

$$
\mathcal{D} \epsilon=0 .
$$

A linear constraint datum for $(S, \Gamma)$ is a pair $(\mathcal{W}, \mathcal{Q})$, where $\mathcal{W}$ is a real vector bundle over $M$ and $\mathcal{Q} \in \Gamma(\operatorname{End}(S) \otimes \mathcal{W}) \simeq \Gamma(\operatorname{Hom}(S, S \otimes \mathcal{W}))$. Given such a datum, the condition:

$$
\mathcal{Q}(\epsilon)=0
$$

is called the linear constraint on $\epsilon$ defined by $\mathcal{Q}$. We say that $\epsilon$ is a (real) constrained generalized Killing spinor if it satisfies the system formed by (53) and (54).

Remark 4.16 Supersymmetric solutions of supergravity theories can often be characterized as manifolds admitting certain systems of generalized constrained Killing spinors, see for instance $[63,66]$. This extends the notion of generalized Killing spinor considered $[9,33,34$, 78].

Suppose that $(M, g)$ is strongly spin and $(S, \Gamma)$ is a strong real spinor bundle. Then we can write $\mathcal{D}=\nabla^{S}-\mathcal{A}$ with $\mathcal{A} \in \Omega^{1}(\operatorname{End}(S))$, where $\nabla^{S}$ is the spinorial connection on $S$. In this case, the equations satisfied by a constrained generalized Killing spinor can be written as:

$$
\nabla^{S} \epsilon=\mathcal{A} \epsilon, \quad \mathcal{Q}(\epsilon)=0
$$

and their solutions are called constrained generalized Killing spinors relative to $(\mathcal{A}, \mathcal{W}, \mathcal{Q})$. When $\mathcal{A}$ is given, we sometimes denote $\mathcal{D}$ by $\mathcal{D}_{\mathcal{A}}$. Using connectedness of $M$ and the parallel transport of $\mathcal{D}$, Eq. (53) implies that the space of constrained generalized Killing spinors relative to $(\mathcal{A}, \mathcal{Q}, \mathcal{W})$ is finite-dimensional and that a constrained generalized Killing spinor which is not zero at some point of $M$ is automatically nowhere-vanishing on $M$; in this case, we say that $\epsilon$ is nontrivial.

\subsection{Spinor squaring maps}

Let $\mathbf{S}=(S, \Gamma, \mathscr{B})$ be a paired spinor bundle on $(M, g)$. The admissible pairing $\mathscr{B}$ of $(S, \Gamma)$ allows us to construct extensions to $M$ of the squaring maps $\mathcal{E}_{ \pm}: \Sigma \rightarrow \operatorname{End}(\Sigma)$ of Sect. 2 and of the spinor squaring maps $\mathcal{E}_{\Sigma}^{ \pm}: \Sigma \rightarrow \wedge V^{*}$ of Sect. 3. We denote these by:

$$
\mathcal{E}_{ \pm}: S \rightarrow \operatorname{End}(S) \text { and } \mathcal{E}_{\mathbf{S}}^{ \pm}: S \rightarrow \Lambda(M) .
$$

Although $\mathcal{E}_{\mathbf{S}}^{ \pm}$preserve fibers, they are not morphisms of vector bundles since they are fiberwise quadratic. By the results of Sect. 3, these maps are two to one away from the zero section of $S$ (where they branch) and their images - which we denote by $Z^{ \pm}(M)$-are subsets of the total space of $\Lambda(M)$ which fiber over $M$ with cone fibers $Z_{m}^{ \pm}(M)(m \in M)$. We have $Z^{-}(M)=-Z^{+}(M)$ and $Z^{+}(M) \cap Z^{-}(M)=0_{\Lambda(M)}$. The fiberwise sign action of $\mathbb{Z}_{2}$ on $S$ 
permutes the sheets of these covers (fixing the zero section), hence $\mathcal{E}_{\mathbf{S}}^{ \pm}$give bijections from $S / \mathbb{Z}_{2}$ to $Z^{ \pm}(M)$ as well as a single bijection:

$$
\hat{\mathcal{E}}_{\mathbf{S}}: S / \mathbb{Z}_{2} \stackrel{\sim}{\rightarrow} Z(M) / \mathbb{Z}_{2}
$$

where $Z(M) \stackrel{\text { def. }}{=} Z^{+}(M) \cup Z^{-}(M)$ and $\mathbb{Z}_{2}$ acts by sign multiplication. The sets $\dot{Z}^{ \pm}(M) \stackrel{\text { def. }}{=}$ $Z^{ \pm}(M) \backslash 0_{\Lambda(M)}$ are connected submanifolds of the total space of $\Lambda(M)$ and the restrictions:

$$
\dot{\mathcal{E}}_{\mathbf{S}}^{ \pm}: \dot{S} \rightarrow \dot{Z}^{ \pm}(M)
$$

of $\mathcal{E}_{\mathbf{S}}^{ \pm}$away from the zero section are surjective morphisms of fiber bundles which are two to one.

Definition 4.17 The signed spinor squaring maps of the paired spinor bundle $\mathbf{S}=(S, \Gamma, \mathscr{B})$ are the maps $\mathcal{E}_{\mathrm{S}}^{ \pm}: \Gamma(S) \rightarrow \Omega(M)$ induced by $\mathcal{E}_{\mathbf{S}}^{ \pm}$on sections (which we denote by the same symbols).

By the results of Sect. $3, \mathcal{E}_{\mathbf{S}}^{ \pm}$are quadratic maps of $\mathcal{C}^{\infty}(M)$-modules and satisfy:

$$
\operatorname{supp}\left(\mathcal{E}_{\mathbf{S}}^{ \pm}(\epsilon)\right)=\operatorname{supp}(\epsilon) \quad \forall \epsilon \in \Gamma(S) .
$$

Let $\mathfrak{Z}^{ \pm}(M) \stackrel{\text { def. }}{=} \mathcal{E}_{\mathbf{S}}^{ \pm}(\Gamma(S)) \subset \Omega(M)$ denote their images and set $\mathfrak{Z}(M) \stackrel{\text { def. }}{=} \mathfrak{Z}^{+}(M) \cup$ $\mathfrak{Z}^{-}(M)$. Then $\mathfrak{Z}^{-}(M)=-\mathfrak{Z}^{+}(M)$ and $\mathfrak{Z}^{+}(M) \cap \mathfrak{Z}^{-}(M)=\{0\}$ and we have strict inclusions $\mathfrak{Z}^{ \pm}(M) \subset \Gamma\left(Z^{ \pm}(M)\right)$ and $\mathfrak{Z}(M) \subset \Gamma(Z(M))$ (see Eq. (5) for notation). Moreover, $\mathcal{E}_{\mathbf{S}}^{ \pm}$ induce the same bijection:

$$
\hat{\mathcal{E}}_{\mathbf{S}}: \Gamma(S) / \mathbb{Z}_{2} \stackrel{\sim}{\rightarrow} \mathfrak{Z}(M) / \mathbb{Z}_{2}
$$

Finally, let $\dot{\Gamma}(S)=\Gamma(\dot{S})$ be the set of nowhere-vanishing sections of $S$ and $\dot{\mathfrak{Z}}^{ \pm}(M) \stackrel{\text { def. }}{=}$ $\dot{\Gamma}\left(Z^{ \pm}(M)\right)=\Gamma(\dot{\mathfrak{Z}}(M)) \subset \mathfrak{Z}^{ \pm}(M)$ be the set of those polyforms in $\mathfrak{Z}^{ \pm}(M)$ which are nowhere-vanishing and define $\dot{\mathfrak{Z}}(M) \stackrel{\text { def. }}{=} \dot{\mathfrak{Z}}^{+}(M) \cup \dot{\mathfrak{Z}}^{-}(M)$. Notice that $\dot{\mathfrak{Z}}^{+}(M) \cap \dot{\mathfrak{Z}}^{-}(M)=\emptyset$. The signed spinor squaring maps restrict to two-to one surjections which coincide with the maps induced by (55) on sections:

$$
\dot{\mathcal{E}}_{\mathbf{S}}^{ \pm}: \dot{\Gamma}(S) \rightarrow \dot{\mathfrak{Z}}^{ \pm}(M) .
$$

Proposition 4.18 Suppose that $(M, g)$ is strongly spin let $S=(S, \Gamma, \mathscr{B})$ be a strong paired spinor bundle associated to a $\operatorname{Spin}_{0}\left(V^{*}, h^{*}\right)$-structure $\mathfrak{Q}$ on $(M, g)$. Then every nowherevanishing polyform $\alpha \in \mathfrak{Z}(M)$ determines a cohomology class $c_{\mathfrak{Q}}(\alpha) \in H^{1}\left(M, \mathbb{Z}_{2}\right)$ encoding the obstruction to existence of a globally-defined spinor $\epsilon \in \Gamma(S)$ (which is necessarily nowhere-vanishing) such that $\alpha \in\left\{\mathcal{E}_{S}^{+}(\epsilon), \mathcal{E}_{S}^{-}(\epsilon)\right\}$. More precisely, such $\epsilon$ exists iff $c_{\mathfrak{Q}}(\alpha)=0$. In particular, we have:

$$
\dot{\mathfrak{Z}}(M)=\left\{\alpha \in \mathfrak{Z}(M) \mid c_{\mathfrak{Q}}(\alpha)=0\right\} \text { and } \dot{\mathfrak{Z}}^{ \pm}(M)=\left\{\alpha \in \mathfrak{Z}^{ \pm}(M) \mid c_{\mathfrak{Q}}(\alpha)=0\right\} .
$$

Proof We have $\alpha \in \mathfrak{Z}^{\kappa}(M)$ for some $\kappa \in\{-1,1\}$. Let $L_{\alpha}$ be the real line sub-bundle of $\Lambda(M)$ determined by $\alpha$. Since the projective spinor squaring map $\mathbb{P} \mathcal{E}_{\mathbf{S}}: \mathbb{P}(S) \rightarrow \mathbb{P}(\wedge(M))$ is bijective, $L_{\alpha}$ determines a real line sub-bundle $L_{\mathfrak{Q}}(\alpha) \stackrel{\text { def. }}{=}\left(\mathbb{P} \mathcal{E}_{\mathbf{S}}\right)^{-1}\left(L_{\alpha}\right)$ of $S$. A section $\epsilon$ of $S$ such that $\mathcal{E}_{\mathbf{S}}^{\kappa}(\epsilon)=\alpha$ is a section of $L_{\mathfrak{Q}}(\alpha)$. Since such $\epsilon$ must be nowhere-vanishing (because $\alpha$ is), it exists iff $L_{\mathfrak{Q}}(\alpha)$ is trivial, which happens iff its first Stiefel-Whitney class 
vanishes. The conclusion follows by setting $c_{\mathfrak{Q}}(\alpha) \stackrel{\text { def. }}{=} w_{1}\left(L_{\mathfrak{Q}}(\alpha)\right) \in H^{1}\left(M, \mathbb{Z}_{2}\right)$. Notice that $c_{\mathfrak{Q}}(\alpha)$ depends only on $\alpha$ and $\mathfrak{Q}$, since the Clifford bundle $(S, \Gamma)$ is associated to $\mathfrak{Q}$ while all admissible pairings of $(S, \Gamma)$ are related to each other by automorphisms of $S$ (see Remark 3.4 in Sect. 3).

Definition 4.19 The cohomology class $c_{\mathfrak{Q}}(\alpha) \in H^{1}\left(M, \mathbb{Z}_{2}\right)$ of the previous proposition is called the spinor class of the nowhere-vanishing polyform $\alpha \in \mathfrak{Z}(M)$.

Remark 4.20 $c_{\mathfrak{Q}}(\alpha)$ is not a characteristic class of $S$, since it depends on $\alpha$.

Lemma 4.21 Let $S=(S, \Gamma, \mathscr{B})$ be a paired real spinor bundle on $(M, g),\left(S_{L}, \Gamma_{L}\right)$ be the modification of $(S, \Gamma)$ by a real line bundle $L$ on $M$ and $q: L^{\otimes 2} \simeq \mathbb{R}_{M}$ be an isomorphism of line bundles. Let $\mathscr{B}_{L}$ be the bilinearnon-degenerate pairing on $S_{L}$ whose duality isomorphism $*_{L}: S_{L} \rightarrow S_{L}^{*}$ satisfies:

$$
*_{L} \otimes I d_{S_{L}}=\varphi_{L}^{-1} \circ\left(* \otimes I d_{S}\right) \circ \psi_{q},
$$

where $*: S \rightarrow S^{*}$ is the duality isomorphism of $\mathscr{B}, \varphi_{L}: \operatorname{End}\left(S_{L}\right) \rightarrow \operatorname{End}(S)$ is the natural isomorphism of bundles of unital algebras and $\psi_{q} \stackrel{\text { def. }}{=} I d_{S \otimes S} \otimes q: S_{L} \otimes S_{L} \rightarrow S \otimes S$ is the isomorphism of vector bundles induced by $q$. Then $\mathscr{B}_{L}$ is an admissible pairing on $\left(S_{L}, \Gamma_{L}\right)$ which has the same symmetry and adjoint type as $\mathscr{B}$. Hence the triplet $S_{L} \stackrel{\text { def. }}{=}\left(S_{L}, \Gamma_{L}, \mathscr{B}_{L}\right)$ is a paired spinor bundle on $(M, g)$ which we call the modification of $S$ by $L$.

Proof Recall from Proposition 4.4 that $\Gamma_{L}=\varphi_{L}^{-1} \circ \Gamma$. A simple computation gives:

$$
\mathscr{B}_{L}\left(\xi_{1} \otimes l_{1}, \xi_{2} \otimes l_{2}\right)=q\left(l_{1} \otimes l_{2}\right) \mathscr{B}\left(\xi_{1}, \xi_{2}\right) \quad \forall \xi_{1}, \xi_{2} \in \Gamma(S) \forall l_{1}, l_{2} \in \Gamma(L),
$$

which immediately implies the conclusion.

The following proposition shows that $c_{\mathfrak{Q}}(\alpha)$ can be made to vanish by changing $\mathfrak{Q}$.

Proposition 4.22 Suppose that $(M, g)$ is strongly spin and a let $\mathfrak{Q}$ be a $\operatorname{Spin}_{0}\left(V^{*}, h^{*}\right)$ structure on $(M, g)$. For every nowhere-vanishing polyform $\alpha \in \mathfrak{Z}(M)$, there exists a unique $\operatorname{Spin}_{0}\left(V^{*}, h^{*}\right)$-structure $\mathfrak{Q}^{\prime}$ such that $c_{\mathfrak{Q}^{\prime}}(\alpha)=0$.

Proof Suppose for definiteness that $\alpha \in \mathfrak{Z}^{+}(M)$. Let $(S, \Gamma)$ be the strong real spinor bundle associated to $\mathfrak{Q}$ and set $L \stackrel{\text { def. }}{=} L_{\mathfrak{Q}}^{+}(\alpha) \subset S$. By Remark 4.10, isomorphism classes of $\operatorname{Spin}_{0}\left(V^{*}, h^{*}\right)$-structures on $(M, g)$ form a torsor over $H^{1}\left(M, \mathbb{Z}_{2}\right)$. Let $\mathfrak{Q}^{\prime}=c_{\mathfrak{Q}}(\alpha) \cdot \mathfrak{Q}$ be the spin structure obtained from $\mathfrak{Q}$ by acting in this torsor with $c_{\mathfrak{Q}}(\alpha)$. Then the strong real spinor bundle associated to $\mathfrak{Q}^{\prime}$ coincides with $\left(S_{L}, \Gamma_{L}\right)$. Pick an isomorphism $q: L^{\otimes 2} \simeq \mathbb{R}_{M}$ and equip $S_{L}$ with the admissible pairing $\mathscr{B}_{L}$ induced from $\mathscr{B}$ by $q$ as in Lemma 4.21. Since $\Psi_{\Gamma_{L}}=\varphi_{L}^{-1} \circ \Psi_{\Gamma}$, relation (56) implies that the polarizations $\mathcal{E}_{\mathbf{S}_{L}}^{+}=\Psi_{\Gamma_{L}}^{-1} \circ\left(*_{L} \otimes \operatorname{Id}_{S_{L}}\right)$ and $\mathcal{E}_{\mathbf{S}}^{+}=\Psi_{\Gamma}^{-1} \circ\left(* \otimes \mathrm{Id}_{S}\right)$ of the positive spinor squaring maps of $\mathbf{S}_{L}$ and $\mathbf{S}$ are related through:

$$
\mathcal{E}_{\mathbf{S}_{L}}^{+}=\mathcal{E}_{\mathbf{S}}^{+} \circ \psi_{q} .
$$

Since $\psi_{q}\left(L^{\otimes 2} \otimes L^{\otimes 2}\right)=L^{\otimes 2}$ (where $L^{\otimes 2}$ is viewed as a sub-bundle of $S_{L}=S \otimes L$ ), this gives $\mathcal{E}_{\mathbf{S}_{L}}^{+}\left(L^{\otimes 2} \otimes L^{\otimes 2}\right)=\mathcal{E}_{\mathbf{S}}^{+}(L \otimes L)$, which implies $\mathcal{E}_{\mathbf{S}_{L}}^{+}\left(L^{\otimes 2}\right)=\mathcal{E}_{\mathbf{S}}^{+}(L)=L_{\alpha}$ Hence the line sub-bundle of $S_{L}$ determined by $\alpha$ is the trivializable real line bundle $L^{\otimes 2} \simeq \mathbb{R}_{M}$. Thus $c_{\mathfrak{Q}^{\prime}}(\alpha)=0$. 


\subsection{Description of constrained generalized Killing spinors as polyforms}

Let $\mathbf{S}=(S, \Gamma, \mathscr{B})$ be a paired spinor bundle (with $\mathscr{B}$ of adjoint type $s)$ and $(\mathcal{W}, \mathcal{Q})$ be a constraint datum for $(S, \Gamma)$. Let $\hat{\mathcal{Q}} \stackrel{\text { def. }}{=}\left(\Psi_{\Gamma} \otimes \operatorname{Id}_{\mathcal{W}}\right) \in \Omega^{*}(M, \mathcal{W})$ be the symbol of $\mathcal{Q}$ (see Definition 4.6). Proposition 3.26 implies:

Lemma 4.23 A spinor $\epsilon \in \Gamma(S)$ satisfies:

$$
\mathcal{Q}(\epsilon)=0
$$

iff one (and hence both) of the following mutually-equivalent relations holds:

$$
\hat{\mathcal{Q}} \diamond \alpha=0, \quad \alpha \diamond\left(\pi^{\frac{1-s}{2}} \circ \tau\right)(\hat{Q})=0,
$$

where $\alpha \stackrel{\text { def. }}{=} \mathcal{E}_{S}^{+}(\epsilon) \in \Omega(M)$ is the positive polyform square of $\epsilon$.

Now assume that $(M, g)$ is strongly spin and that $(S, \Gamma, \mathscr{B})$ is the paired spinor bundle associated to a $\operatorname{Spin}_{0}\left(V^{*}, h^{*}\right)$-structure. Set $\mathcal{A} \stackrel{\text { def. }}{=} \nabla^{S}-\mathcal{D} \in \Omega^{1}(M, \operatorname{End}(S))$ and let $\hat{\mathcal{A}} \stackrel{\text { def. }}{=}\left(\Psi_{\Gamma} \otimes \operatorname{Id}_{T^{*} M}\right)^{-1}(\mathcal{A}) \in \Omega^{1}(M, \Lambda(M))$ be the symbol of $\mathcal{A}$, viewed as a $\Lambda(M)$-valued one-form. In this case, we have:

Lemma 4.24 A nowhere-vanishing spinor $\epsilon \in \Gamma(S)$ satisfies $\mathcal{D} \epsilon=0$ iff:

$$
\nabla^{g} \alpha=\hat{\mathcal{A}} \diamond \alpha+\alpha \diamond\left(\pi^{\frac{1-s}{2}} \circ \tau\right)(\hat{\mathcal{A}}),
$$

where $\alpha \stackrel{\text { def. }}{=} \mathcal{E}_{S}^{+}(\epsilon)$ is the positive polyform square of $\epsilon$.

Proof Assume that $\epsilon$ satisfies $\nabla^{S} \epsilon=\mathcal{A}(\epsilon)$. We have $\alpha \in \Gamma(\operatorname{End}(S))$ and:

$$
\begin{aligned}
D^{S}\left(\mathcal{E}_{\mathbf{S}}^{+}(\epsilon)\right)(\chi) & =\nabla^{S}\left(\mathcal{E}_{\mathbf{S}}^{+}(\epsilon)\right)(\chi)-\mathcal{E}_{\mathbf{S}}^{+}(\epsilon)\left(\nabla^{S} \chi\right) \\
& =\nabla^{S}(\mathscr{B}(\chi, \epsilon) \epsilon)-\mathscr{B}\left(\nabla^{S} \chi, \epsilon\right) \epsilon \\
& =\mathscr{B}\left(\chi, \nabla^{S} \epsilon\right) \epsilon+\mathscr{B}(\chi, \epsilon) \nabla^{S} \epsilon=\mathscr{B}(\chi, \mathcal{A} \epsilon) \epsilon+\mathscr{B}(\chi, \epsilon) \mathcal{A} \epsilon \\
& =\mathcal{E}_{\mathbf{S}}^{+}(\epsilon)\left(\mathcal{A}^{t} \chi\right)+\mathcal{A}\left(\mathcal{E}_{\mathbf{S}}^{+}(\epsilon)\right)(\chi)
\end{aligned}
$$

for all $\chi \in \Gamma(S)$, where $\mathcal{A}^{t}$ is obtained by fiberwise application of the $\mathscr{B}$-transpose of Lemma 3.15. The equation above implies:

$$
D^{S}\left(\mathcal{E}_{\mathbf{S}}^{+}(\epsilon)\right)=\mathcal{A} \circ \mathcal{E}_{\mathbf{S}}^{+}(\epsilon)+\mathcal{E}_{\mathbf{S}}^{+}(\epsilon) \circ \mathcal{A}^{t} .
$$

Applying $\Psi_{\Gamma}^{-1}$ and using Lemma 3.15 and Proposition 4.1 gives (57).

Conversely, assume that $\alpha$ satisfies (57). Applying $\Psi_{\Gamma}$ gives Eq. (58), which reads:

$$
\mathscr{B}\left(\chi, \mathcal{D}_{X} \epsilon\right) \epsilon+\mathscr{B}(\chi, \epsilon) \mathcal{D}_{X} \epsilon=0 \quad \forall \chi \in \Gamma(S) \forall X \in \mathfrak{X}(M) .
$$

Hence $\mathcal{D}_{X} \epsilon=\beta(X) \epsilon$ for some $\beta \in \Omega^{1}(M)$. Using this in (59) gives:

$$
\mathscr{B}(\chi, \epsilon) \beta \otimes \epsilon=0 \quad \forall \chi \in \Gamma(S) .
$$

This implies $\beta=0$, since $\mathscr{B}$ is non-degenerate and $\epsilon$ is nowhere-vanishing. Hence $\mathcal{D} \epsilon=0$.

Remark 4.25 If $\mathcal{A}$ is skew-symmetric with respect to $\mathscr{B}$, then (57) simplifies to:

$$
\nabla^{g} \alpha=\hat{\mathcal{A}} \diamond \alpha-\alpha \diamond \hat{\mathcal{A}} \text {. }
$$

In applications to supergravity, $\mathcal{A}$ need not be skew-symmetric relative to $\mathscr{B}$. 
Theorem 4.26 Suppose that $(M, g)$ is strongly spin and let $S=(S, \Gamma, \mathscr{B})$ be a paired spinor bundle associated to the $\operatorname{Spin}_{0}\left(V^{*}, h^{*}\right)$-structure $\mathfrak{Q}$ and whose admissible form $\mathscr{B}$ has adjoint type s. Let $\mathcal{A} \in \Omega^{1}(M, \operatorname{End}(S))$ and $(\mathcal{W}, Q)$ be a linear constraint datum for $(S, \Gamma)$. Then the following statements are equivalent:

(a) There exists a nontrivial generalized constrained Killing spinor $\epsilon \in \Gamma(S)$ with respect to $(\mathcal{A}, \mathcal{W}, \mathcal{Q})$.

(b) There exists a nowhere-vanishing polyform $\alpha \in \Omega(M)$ with vanishing cohomology class $c_{\mathfrak{Q}}(\alpha)$ which satisfies the following algebraic and differential equations for every polyform $\beta \in \Omega(M)$ :

$$
\begin{aligned}
& \alpha \diamond \beta \diamond \alpha=\mathcal{S}(\alpha \diamond \beta) \alpha, \quad\left(\pi^{\frac{1-s}{2}} \circ \tau\right)(\alpha)=\sigma_{s} \alpha, \\
& \nabla^{g} \alpha=\hat{\mathcal{A}} \diamond \alpha+\alpha \diamond\left(\pi^{\frac{1-s}{2}} \circ \tau\right)(\hat{\mathcal{A}}), \quad \hat{\mathcal{Q}} \diamond \alpha=0
\end{aligned}
$$

or, equivalently, satisfies the equations:

$$
\begin{gathered}
\alpha \diamond \alpha=\mathcal{S}(\alpha) \alpha, \quad\left(\pi^{\frac{1-s}{2}} \circ \tau\right)(\alpha)=\sigma_{s} \alpha, \quad \alpha \diamond \beta \diamond \alpha=\mathcal{S}(\alpha \diamond \beta) \alpha, \\
\nabla^{g} \alpha=\hat{\mathcal{A}} \diamond \alpha+\alpha \diamond\left(\pi^{\frac{1-s}{2}} \circ \tau\right)(\hat{\mathcal{A}}), \quad \hat{\mathcal{Q}} \diamond \alpha=0,
\end{gathered}
$$

for some fixed polyform $\beta \in \Omega(M)$ such that $\mathcal{S}(\alpha \diamond \beta) \neq 0$.

If $\epsilon \in \Gamma(S)$ is chiral of chirality $\mu \in\{-1,1\}$, then we have to add the condition:

$$
*(\pi \circ \tau)(\alpha)=\mu \alpha .
$$

The polyform $\alpha$ as above is determined by $\epsilon$ through the relation:

$$
\alpha=\mathcal{E}_{S}^{\kappa}(\epsilon)
$$

for some $\kappa \in\{-1,1\}$. Moreover, $\alpha$ satisfying the conditions above determines a nowherevanishing real spinor $\epsilon$ satisfying this relation, which is unique up to sign.

Remark 4.27 Suppose that $\alpha \in \Omega(M)$ is nowhere-vanishing and satisfies (61) and (62) but we have $c_{\mathfrak{Q}}(\alpha) \neq 0$. Then Proposition 4.22 implies that there exists a unique $\operatorname{Spin}_{0}\left(V^{*}, h^{*}\right)$ structure $\mathfrak{Q}^{\prime}$ such that $c_{\mathfrak{Q}^{\prime}}(\alpha)=0$. Thus $\alpha$ is the square of a global section of a paired spinor bundle $\left(S^{\prime}, \Gamma^{\prime}, \mathscr{B}^{\prime}\right)$ associated to $\mathfrak{Q}^{\prime}$. Hence a nowhere-vanishing polyform $\alpha$ satisfying (61) and (62) corresponds to the square of a generalized Killing spinor with respect to a uniquelydetermined $\operatorname{Spin}_{0}\left(V^{*}, h^{*}\right)$-structure.

Proof The algebraic conditions in the Theorem follow from the pointwise extension of Theorem 3.20 and Corollary 3.28. The differential condition follows from Lemma 4.24, which implies that $\mathcal{D}_{\mathcal{A}} \epsilon=0$ holds iff (62) does upon noticing that $\epsilon \in \Gamma(S)$ vanishes at a point $m \in M$ iff its positive polyform square $\alpha$ satisfies $\left.\alpha\right|_{m}=0$. The condition $c_{\mathfrak{Q}}(\alpha)=0$ follows from Proposition 4.18.

In Sects. 5 and 6, we apply this theorem to real Killing spinors on Lorentzian fourmanifolds and to supersymmetric configurations of heterotic supergravity on principal bundles over such manifolds. 


\subsection{Real spinors on Lorentzian four-manifolds}

Let $(M, g)$ be a spin Lorentzian four-manifold of "mostly plus" signature such that $H^{1}\left(M, \mathbb{Z}_{2}\right)=0$. By Remark 4.10 , this condition insures that $(M, g)$ is strongly spin, with a $\operatorname{Spin}\left(V^{*}, h^{*}\right)$-structure, $\operatorname{Spin}_{0}\left(V^{*}, h^{*}\right)$-structure and real spinor bundle $(S, \Gamma)$ which are unique up to isomorphism. Let $\mathcal{S}=(S, \Gamma, \mathscr{B})$, where the admissible pairing $\mathscr{B}$ is skewsymmetric and of negative adjoint type. The spinor class $c_{\mathfrak{Q}}(\alpha)$ (see Definition 4.19) of any nowhere-vanishing polyform $\alpha \in \mathfrak{Z}(M)$ vanishes since $H^{1}\left(M, \mathbb{Z}_{2}\right)=0$. Hence any such $\alpha$ is a signed square of a nowhere-vanishing spinor. We will characterize real spinors on $(M, g)$ through certain pairs of one-forms.

Definition 4.28 A pair of nowhere-vanishing one-forms $(u, l) \in \Omega^{1}(M) \times \Omega^{1}(M)$ is called parabolic if:

$$
g^{*}(u, u)=0, \quad g^{*}(l, l)=1, \quad g^{*}(u, l)=0,
$$

i.e. $u$ and $l$ are mutually-orthogonal, with $u$ timelike and $l$ spacelike of unit norm. Two parabolic pairs of one-forms $(u, l)$ and $\left(u^{\prime}, l^{\prime}\right)$ are called strongly equivalent if there exists a sign factor $\zeta \in\{-1,1\}$ and a real constant $c \in \mathbb{R}$ such that:

$$
u^{\prime}=\zeta u \text { and } l^{\prime}=l+c u .
$$

Let $\mathcal{P}(M, g)$ denote the set of parabolic pairs of one-forms defined on $(M, g)$.

Definition 4.29 A rank two vector sub-bundle $\Pi$ of $T^{*} M$ is called a distribution of parabolic 2-planes in $T^{*} M$ if, for all $m \in M$, the fiber $\Pi_{m}$ is a parabolic 2-plane in the Minkowski space $\left(T_{m}^{*} M, g_{m}^{*}\right)$.

Definition 4.30 Let $\Pi$ is a distribution of parabolic 2-planes in $T^{*} M$. The real line sub-bundle $\mathrm{K}_{h}(\Pi) \stackrel{\text { def. }}{=} \operatorname{ker}\left(g_{\Pi}^{*}\right)$ (where $g_{\Pi}^{*}$ is the restriction of $g^{*}$ to $\Pi$ ) is called the null line sub-bundle of $\Pi$ and $\Pi$ is called co-orientable if the quotient line bundle $N_{h}(\Pi) \stackrel{\text { def. }}{=} \Pi / \mathrm{K}_{h}(\Pi)$ is trivializable. In this case, a co-orientation of $\Pi$ is an orientation of $\Pi / K_{h}(\Pi)$.

A co-orientation of $\Pi$ amounts to the choice of a sub-bundle of half-planes $\mathcal{H} \subset \Pi$ such that $\mathcal{H}_{m}$ is one of the two spacelike half-planes of $\Pi_{m}$ for each $m \in M$. In this case, the pair $(\Pi, \mathcal{H})$ is called a co-oriented distribution of parabolic 2-planes in $T^{*} M$.

Definition 4.31 Let $\Pi$ be a distribution of parabolic 2-planes in $T^{*} M$. A local frame $(u, l)$ of $\Pi$ defined on a non-empty open subset $U \subset V$ is called a local parabolic frame if $(u, l)$ is a parabolic pair of one-forms for the Lorenzian manifold $\left(U,\left.g\right|_{U}\right)$. Such a frame is called global if $U=M$.

Local parabolic frames of $\Pi$ defined above $U$ are determined up to transformations of the form:

$$
u^{\prime}=b u \text { and } l^{\prime}=\zeta l+c u,
$$

where $\zeta \in\{-1,1\}$ and $b, c$ are nowhere-vanishing smooth functions defined on $U$. Notice that $\Pi$ admits a global parabolic frame iff it is trivializable. Since $H^{1}\left(M, \mathbb{Z}_{2}\right)=0$, any smooth section of the projective bundle $\mathbb{P}(S)$ lifts to a nowhere-vanishing section of $S$ and hence we have:

$$
\Gamma(\mathbb{P}(S))=\Gamma(\dot{S}) / \mathcal{C}^{\infty}(M)^{\times}
$$


where the multiplicative group $\mathcal{C}^{\infty}(M)^{\times}$of nowhere-vanishing real-valued functions defined on $M$ acts on $\Gamma(S)$ through multiplication of sections by the corresponding function. The results of Sect. 3.6.3 imply:

Theorem 4.32 There exists a natural bijection between the set $\Gamma(\mathbb{P}(S))=\Gamma(\dot{S}) / \mathcal{C}^{\infty}(M)^{\times}$ and the set of trivializable and co-oriented distributions $(\Pi, \mathcal{H})$ of parabolic 2-planes in $T^{*} M$. Moreover, there exist natural bijections between the following two sets:

(a) The set $\Gamma(\dot{S}) / \mathbb{Z}_{2}$ of sign-equivalence classes of nowhere-vanishing real spinors $\epsilon \in$ $\Gamma(S)$.

(b) The set of strong equivalence classes of parabolic pairs of one-forms $(u, l) \in \mathcal{P}(M, g)$.

Remark 4.33 Let $(u, l)$ be a parabolic pair of one-forms corresponding to a nowherevanishing spinor $\xi \in \Gamma(S)$. Then $\alpha \stackrel{\text { def. }}{=} u+u \wedge l$ is a signed polyform square of $\xi$ by Sect. 3.6.3.

\subsection{Real spinors on globally hyperbolic Lorentzian four-manifolds}

Let $(M, g)$ be an oriented and spin Lorentzian four-manifold of "mostly plus" signature such that $H^{1}\left(M, \mathbb{Z}_{2}\right)=0$. As before, let $\mathbf{S}=(S, \Gamma, \mathscr{B})$ be a paired real spinor bundle on $(M, g)$, where $\mathscr{B}$ is skew-symmetric and of negative adjoint type.

Proposition 4.34 Let $v \in \Omega^{1}(M)$ be a timelike one-form such that $g^{*}(v, v)=-1$. Let $P_{v}: T^{*} M \rightarrow L_{v}$ be the orthogonal projection onto the real line sub-bundle $L_{v}$ of $T^{*} M$ determined by $v$. For any parabolic pair $(u, l)$ on $(M, g)$, there exists a unique smooth function $f \in C^{\infty}(M)$ such that:

$$
P_{v}(l+f u)=0 .
$$

Moreover, there exist exactly two parabolic pairs of one-forms $\left(u^{\prime}, l^{\prime}\right)$ which are stronglyequivalent with $(u, l)$ and satisfy $P_{v}\left(l^{\prime}\right)=0$, namely:

$$
u^{\prime}=u, l^{\prime}=l+f u \text { and } u^{\prime}=-u, l^{\prime}=l+f u
$$

and every pair $\left(u^{\prime \prime}, l^{\prime \prime}\right)$ which is equivalent with $(u, l)$ and satisfies $P_{v}\left(l^{\prime \prime}\right)=0$ has the form:

$$
u^{\prime \prime}=b u, \quad l^{\prime \prime}=l+f u
$$

where $b \in \mathcal{C}^{\infty}(M)^{\times}$is a nowhere-vanishing smooth function.

Proof We have:

$$
P_{v}(\alpha)=-g^{*}(\alpha, v) v, \quad \forall \alpha \in \Omega^{1}(M) .
$$

Notice that $g^{*}(u, v) \neq 0$ since $u \neq 0$ is lightlike and $v$ is timelike. Condition (65) amounts to:

$$
g^{*}(l, v)+f g^{*}(u, v)=0,
$$

which is solved by $f=-\frac{g^{*}(l, v)}{g^{*}(u, v)}$. The remaining statements follow immediately from the definition of equivalence and strong equivalence of parabolic pairs (see Definition 3.30).

Let $\mathcal{P}_{v}(M, g)$ denote the set of parabolic pairs of one-forms $(u, l)$ on $(M, g)$ which satisfy $P_{v}(l)=0$. The group $\mathbb{Z}_{2}$ acts on this set by changing the sign of $u$ while leaving $l$ unchanged. Proposition 4.34 and Theorem 4.32 imply: 
Corollary 4.35 With the assumptions of the previous proposition, there exists a bijection between the sets $\Gamma(\dot{S}) / \mathbb{Z}_{2}$ and $\mathcal{P}_{v}(M, g) / \mathbb{Z}_{2}$.

Assume next that $(M, g)$ is globally hyperbolic. By a theorem of Bernal and Sánchez [14], it follows that $(M, g)$ is isometric to $\mathbb{R} \times N$ equipped with the warped product metric $g=-F \mathrm{~d} t \otimes \mathrm{d} t+k(t)$, where $N$ is an oriented three-manifold, $F \in C^{\infty}(\mathbb{R} \times N)$ is a strictly positive function and $k(t)$ is a Riemannian metric on $N$ for every $t \in \mathbb{R}$. Let $V_{2}(N, k(t))$ be the bundle of ordered orthonormal pairs of one-forms on $N$. Since $N$ is oriented, any element of $V_{2}(N, k(t))$ determines an element of the principal bundle $P_{\mathrm{SO}(3)}(N, k(t))$ of oriented frames of $(N, k(t))$, showing that $V_{2}(N, k(t))$ is a principal $\mathrm{SO}(3)$-bundle. Let $\mathcal{V}_{2}$ be the fiber bundle defined on $M=\mathbb{R} \times N$ whose fiber at $(t, n) \in \mathbb{R} \times M$ is given by:

$$
\mathcal{V}_{2}(t, n) \stackrel{\text { def. }}{=} V_{2}\left(T_{n}^{*} N, k(t)_{n}\right),
$$

where $V_{2}(N, k(t))_{n}=V_{2}\left(T_{n}^{*} N, k(t)_{n}\right)$ is the manifold of $k(t)_{n}$-orthonormal systems of two elements of $T_{n}^{*} N$. Consider the fiberwise involution $i_{1}$ of $\mathcal{V}_{2}$ defined through:

$$
i_{1}\left(e_{1}, e_{2}\right) \stackrel{\text { def. }}{=}\left(-e_{1}, e_{2}\right) \quad \forall\left(e_{1}, e_{2}\right) \in \mathcal{V}_{2} \text {. }
$$

and let $\mathbb{Z}_{2}$ act on the set $\mathcal{C}^{\infty}(R \times N)^{\times} \times \Gamma\left(\mathcal{V}_{2}\right)$ through the involution:

$$
(\mathfrak{f}, \mathfrak{s}) \rightarrow\left(-\mathfrak{f}, i_{1}(\mathfrak{s})\right) \quad \forall \mathfrak{f} \in \mathcal{C}^{\infty}(R \times N)^{\times} \quad \forall \mathfrak{s} \in \Gamma\left(\mathcal{V}_{2}\right) .
$$

Proposition 4.36 Consider a globally hyperbolic Lorentzian four-manifold:

$$
(M, g)=(\mathbb{R} \times N,-F d t \otimes d t \oplus k(t))
$$

such that $N$ is oriented and spin and $H^{1}\left(N, \mathbb{Z}_{2}\right)=0$. Then there exists a bijection between the set $\Gamma(\dot{S}) / \mathbb{Z}_{2}$ of sign-equivalence classes of nowhere-vanishing real spinors defined on $M$ and the set $\left[\mathcal{C}^{\infty}(\mathbb{R} \times N)^{\times} \times \Gamma\left(\mathcal{V}_{2}\right)\right] / \mathbb{Z}_{2}$. Moreover, there exists a bijection between the sets $\Gamma(\mathbb{P}(S))$ and $\Gamma\left(\mathcal{V}_{2}\right) / \mathbb{Z}_{2}$, where $\mathbb{Z}_{2}$ acts on $\Gamma\left(\mathcal{V}_{2}\right)$ through the involution $i_{1}$.

Proof Let $v \stackrel{\text { def. }}{=} F^{\frac{1}{2}} \mathrm{~d} t$ and consider a parabolic pair of one-forms $(u, l)$ on $M$ such that $P_{v}(l)=0$. Since $l$ has unit norm and is orthogonal to $v$, it can be viewed as a family of one-forms (parameterized by $t \in \mathbb{R}$ ) defined on $N$. We decompose $u$ orthogonally as:

$$
u=P_{v}(u)+u^{\perp}=-g^{*}(u, v) v \oplus u^{\perp},
$$

where $u^{\perp} \stackrel{\text { def. }}{=} u-P_{v}(u)=u+g^{*}(u, v) v$ and $g^{*}(u, v), g^{*}\left(u^{\perp}, u^{\perp}\right)$ are nowhere-vanishing. The spacelike 1 -form $u^{\perp}$ satisfies:

$$
g^{*}\left(u^{\perp}, u^{\perp}\right)=g^{*}(u, v)^{2} .
$$

Thus $u$ can be written as:

$$
u=\mathfrak{f} v \oplus|\mathfrak{f}| e_{u},
$$

where:

$$
e_{u} \stackrel{\text { def. }}{=} \frac{u^{\perp}}{g^{*}\left(u^{\perp}, u^{\perp}\right)^{\frac{1}{2}}}=\frac{u^{\perp}}{\left|g^{*}(u, v)\right|} \text { and } \mathfrak{f} \stackrel{\text { def. }}{=}-g^{*}(u, v) \in \mathcal{C}^{\infty}(\mathbb{R} \times N) .
$$

The pair $\left(e_{u}, l\right)$ determines an orthonormal pair of one-forms $\left(e_{u}(t), l(t)\right)$ on $(N, k(t))$ for all $t \in \mathbb{R}$, which gives a section $\mathfrak{s}$ of the fiber bundle $\mathcal{V}_{2}$. It is clear that the parabolic pair $(u, l)$ determines and is determined by the pair $(\mathfrak{f}, \mathfrak{s})$. The conclusion now follows from Corollary 4.35 by noticing that the transformation $u \rightarrow b u\left(\right.$ with $b \in \mathcal{C}^{\infty}(M, \mathbb{R})$ ) corresponds to $f \rightarrow b$ f and $e_{u} \rightarrow \operatorname{sign}(b) e_{u}$. 
The three-manifold $N$ is parallelizable since it is connected, oriented and spin. This holds for both compact and open $M$ by considering the Whitehead tower of $\mathrm{BO}(3)$ (the classifying space of $\mathrm{O}(3))$ and using the fact that $\pi_{3}(\mathrm{BSpin}(3))=0$. Hence there exists a unique family $\{e(t)\}_{t \in \mathbb{R}}$ of one-forms on $N$ such that $\left(e_{u}(t), l(t), e(t)\right)$ is an oriented orthonormal global frame of $\left(T^{*} N, k_{t}\right)$ for all $t \in \mathbb{R}$. This produces a parallelization of $(M, g)$ given by $\left(v, e_{u}(t), l(t), e(t)\right)_{t \in \mathbb{R}}$. Let:

$$
R \stackrel{\text { def. }}{=} \operatorname{diag}(-1,0,0) \in \mathrm{SO}(3) .
$$

and let $\mathbb{Z}_{2}$ act on $\mathcal{C}^{\infty}(\mathbb{R} \times N)^{\times} \times \mathcal{C}^{\infty}(N, \mathrm{SO}(3))$ through the involution:

$$
(\mathfrak{f}, \psi) \rightarrow\left(-\mathfrak{f}, \operatorname{Ad}_{R} \circ \psi\right) \quad \forall \mathfrak{f} \in \mathcal{C}^{\infty}(\mathbb{R} \times N)^{\times} \forall \psi \in \mathcal{C}^{\infty}(N, \operatorname{SO}(3)),
$$

where:

$$
\left(\operatorname{Ad}_{R} \circ \psi\right)(t, n)=R \circ \psi(t, n) \circ R^{-1} \quad \forall(t, n) \in \mathbb{R} \times N .
$$

The previous proposition implies:

Corollary 4.37 Let $(M, g)$ be as in Proposition 4.36 and fix a global oriented orthonormal frame of $T^{*} N$. Then there exists a bijection between the set $\Gamma(\dot{S}) / \mathbb{Z}_{2}$ of sign equivalence classes of nowhere-vanishing real spinors defined on $M$ and the set $\left[\mathcal{C}^{\infty}(\mathbb{R} \times\right.$ $\left.N)^{\times} \times \mathcal{C}^{\infty}(N, S O(3))\right] / \mathbb{Z}_{2}$. Moreover, there exists a bijection between the sets $\Gamma(\mathbb{P}(S))$ and $\mathcal{C}^{\infty}(N, S O(3)) / \mathbb{Z}_{2}$, where $\mathbb{Z}_{2}$ acts on $\mathcal{C}^{\infty}(N$, $S O(3))$ through the involution:

$$
\psi \rightarrow A d_{R} \circ \psi \quad \forall \psi \in \mathcal{C}^{\infty}(N, S O(3)) .
$$

We hope that this characterization can be useful in the study of globally hyperbolic Lorentzian four-manifolds admitting spinors satisfying various partial differential equations.

\section{Real Killing spinors on Lorentzian four-manifolds}

Definition 5.1 Let $(M, g)$ be a pseudo-Riemannian manifold which is oriented and strongly spin and $(S, \Gamma)$ be a strong real spinor bundle on $(M, g)$. Let $\lambda \in \mathbb{R}$ be a real number. A real Killing spinor of Killing constant $\frac{\lambda}{2}$ is a global section $\epsilon \in \Gamma(S)$ which satisfies:

$$
\nabla_{X}^{S} \epsilon=\frac{\lambda}{2} X^{b} \cdot \epsilon \quad \forall X \in \mathfrak{X}(M) .
$$

It is called a parallel spinor if $\lambda=0$.

Real Killing spinors are (unconstrained) generalized Killing spinors relative to the connection $\mathcal{D}=\nabla^{S}-\mathcal{A}$ defined on $S$, where $\mathcal{A}_{X}=\frac{\lambda}{2} \Psi_{\Gamma}\left(X^{b}\right) \in \Gamma(E n d(S))$ for all $X \in \mathfrak{X}(M)$. The $\operatorname{End}(S)$-valued one-form $\mathcal{A}$ has symbol $\hat{\mathcal{A}} \in \Omega^{1}\left(M, T^{*} M\right)$ given by $\hat{\mathcal{A}}_{X}=\frac{\lambda}{2} X^{\mathrm{b}}$. In this section, we study real Killing spinors when $(M, g)$ is a spin Lorentzian four-manifold of "mostly plus" signature $(3,1)$ such that $H^{1}\left(M, \mathbb{Z}_{2}\right)=0$.

Remark 5.2 When $p-q \equiv_{8} 0,2$, a real Killing spinor can be viewed as a complex Killing spinor which is preserved by a $\operatorname{Spin}_{0}(p, q)$-invariant real structure on the complex spinor bundle and which has real (in signature $(p, q)$ ) or purely imaginary (in signature $(q, p)$ ) Killing constant. When comparing signatures, note that [15] has a sign in the Clifford relation opposite to our convention (19). In the conventions of loc. cit., the real Killing spinors considered below correspond to special cases of imaginary Killing spinors, which were 
studied in [16,73]. Reference [73] proves that a Lorentzian four-manifold admitting a nontrivial imaginary Killing spinor (which is a real Killing spinor in our convention) with null Dirac current is locally conformal to a Brinkmann space-time ${ }^{7}$. In this section, we give a global characterization of Lorentzian four-manifolds admitting real Killing spinors (see Theorem 5.3).

\subsection{Describing real Killing spinors through differential forms}

For the remainder of this section, let $(M, g)$ be a spin Lorentzian four-manifold of "mostly plus" signature which satisfies $H^{1}\left(M, \mathbb{Z}_{2}\right)=0$. Let $(S, \Gamma)$ be a spinor bundle on $(M, g)$. Since $H^{1}\left(M, \mathbb{Z}_{2}\right)$ vanishes, the spinor bundle is automatically strongly spin and unique up to isomorphism. We endow it with an admissible pairing $\mathscr{B}$ which is is skew-symmetric and of negative adjoint type.

Theorem $5.3(M, g)$ admits a nontrivial real Killing spinor with Killing constant $\frac{\lambda}{2}$ iff it admits a parabolic pair of one-forms $(u, l)$ which satisfies:

$$
\nabla^{g} u=\lambda u \wedge l, \quad \nabla^{g} l=\kappa \otimes u+\lambda(l \otimes l-g)
$$

for some $\kappa \in \Omega^{1}(M)$. In this case, $u^{\sharp} \in \mathfrak{X}(M)$ is a Killing vector field with geodesic integral curves.

Remark 5.4 Our conventions for the wedge product of one-forms are as follows, where $\mathrm{S}_{k}$ denotes the permutation group on $k$ letters:

$$
\theta_{1} \wedge \cdots \wedge \theta_{k} \stackrel{\text { def. }}{=} \sum_{\sigma \in \mathrm{S}_{k}} \epsilon(\sigma) \theta_{\sigma(1)} \otimes \cdots \otimes \theta_{\sigma(k)} .
$$

where $\theta_{1}, \ldots, \theta_{k} \in \Omega^{1}(M)$.

Proof Recall from Sect. 4.6 that a spinor $\xi$ associated to $(u, l)$ has a signed polyform square given by $\alpha=u+u \wedge l$. Theorem 4.26 shows that $\xi$ is a real Killing spinor iff:

$$
\nabla_{X}^{g}(u+u \wedge l)=\hat{\mathcal{A}}_{X} \diamond(u+u \wedge l)+(u+u \wedge l) \diamond(\pi \circ \tau)\left(\hat{\mathcal{A}}_{X}\right) \quad \forall X \in \mathfrak{X}(M),
$$

where $\hat{\mathcal{A}}_{X}=\frac{\lambda}{2} X^{\mathrm{b}}$. Expanding the geometric product and isolating degrees, this equation gives:

$$
\nabla_{X}^{g} u=\lambda(u(X) l-l(X) u), \quad \nabla_{X}^{g}(u \wedge l)=\lambda X^{b} \wedge u,
$$

which in turn amounts to (66) for some $\kappa \in \Omega^{1}(M)$. The vector field $u^{\sharp}$ is Killing since $\nabla^{g} u$ is an antisymmetric covariant 2-tensor by the first equation in (66). Since $u$ is null and orthogonal to $l$, the same equation gives $\nabla_{u^{\sharp}}^{g} u=0$. Hence $\nabla_{u^{\sharp}}^{g} u^{\sharp}=0$, i.e. $u^{\sharp}$ is a geodesic vector field.

Remark 5.5 The first equation in (66) gives:

$$
\nabla_{X}^{g} u^{\sharp}=\lambda\left(u(X) l^{\sharp}-l(X) u^{\sharp}\right) \quad \forall X \in \mathfrak{X}(M) .
$$

Hence the null vector field $u^{\sharp} \in \mathfrak{X}(M)$ is not recurrent, i.e. $\nabla^{g}$ does not preserve the rank one distribution spanned by $u^{\sharp}$. Lorentzian manifolds admitting recurrent vector fields are called almost decent and were studied extensively (see [17,36,37] and references therein).

7 Recall that a Brinkmann space-time is a four-dimensional Lorentzian manifold equipped with a nonvanishing parallel null vector field. 
Taking $\lambda=0$ in Theorem 5.3 gives:

Corollary $5.6(M, g)$ admits a nontrivial parallel real spinor iff it admits a parabolic pair of one-forms $(u, l)$ which satisfies the following conditions for some one-form $\kappa \in \Omega^{1}(M)$ :

$$
\nabla^{g} u=0, \quad \nabla^{g} l=\kappa \otimes u .
$$

Although Lorentzian manifolds admitting parallel spinors were studied extensively in the literature (see $[18,72]$ and references therein), Corollary 5.6 seems to be new. Recall that $u$ coincides up to sign with the Dirac current of any of the spinors $\xi,-\xi$ determined by the parabolic pair $(u, v)$ (see Remark 3.23). Reference [73] shows that a Lorentzian fourmanifold admitting an (imaginary, in the conventions of loc. cit) Killing spinor with null Dirac current is locally conformally Brinkmann. The following proposition recovers this result in our approach.

Proposition 5.7 Suppose that $(M, g)$ admits a nontrivial real Killing spinor with nonzero Killing constant $\frac{\lambda}{2} \neq 0$ and let $(u, l)$ be a corresponding parabolic pair of one-forms. Then $u$ is locally conformally parallel iff $l$ is locally equivalent to a closed one-form $l^{\prime}$ by transformations of the form (70). In this case, $(M, g)$ is locally conformal to a Brinkmann space-time.

Proof The one-form $u$ is locally conformally parallel iff for sufficiently small non-empty open subsets $U \subset M$ there exists $f \in \mathcal{C}^{\infty}(U)$ such that the metric $\hat{g}=e^{f} g$ satisfies $\nabla^{\hat{g}} u=0$ on $U$. This amounts to:

$$
\begin{aligned}
0 & =\nabla_{X}^{\hat{g}} u=\nabla_{X}^{g} u+\mathrm{d} f(X) u+\mathrm{d} f\left(u^{\sharp}\right) X^{b}-u(X) \mathrm{d} f \\
& =\lambda(u(X) l-l(X) u)+\mathrm{d} f(X) u+\mathrm{d} f\left(u^{\sharp}\right) X^{b}-u(X) \mathrm{d} f \quad \forall X \in \mathfrak{X}(U),
\end{aligned}
$$

where in the last equality we used the first equation in (66). Taking $X=u^{\sharp}$ and using the fact that $u$ is nowhere-vanishing, null and orthogonal to $l$ gives $(\mathrm{d} f)\left(u^{\sharp}\right)=0$, whence (68) reduces to:

$$
u \otimes(\mathrm{d} f-\lambda l)=(\mathrm{d} f-\lambda l) \otimes u,
$$

which amounts to the condition $\mathrm{d} f=\lambda(l+c u)$ for some $c \in \mathcal{C}^{\infty}(U)$. This has local solutions $f$ iff $l+c u$ is closed for some locally-defined function $c$. In this case, the nowhere-vanishing null one form $u$ is $\nabla^{\hat{g}}$-parallel and hence $(M, g)$ is locally conformally Brinkmann.

\subsection{The Pfaffian system and its consequences}

Antisymmetrizing the two equations in (66) gives the Pfaffian system:

$$
\mathrm{d} u=2 \lambda u \wedge l, \quad \mathrm{~d} l=\kappa \wedge u,
$$

which implies:

Lemma 5.8 Let $(u, l)$ be a parabolic pair of one-forms which satisfies Eq. (66) for some $\kappa \in \Omega^{1}(M)$ and $\lambda \in \mathbb{R}$ and let $C_{u} \subset T M$ be the rank one distribution spanned by $u^{\sharp}$. Then $l$ is closed if and only iff $\kappa \in \Gamma\left(C_{u}\right)$. Moreover, $u$ is closed iff $\lambda=0$.

Remark 5.9 Let:

$$
l^{\prime}=l+c u,
$$


where $c \in \mathcal{C}^{\infty}(M)$. Then the parabolic pair $(u, l)$ satisfies (66) for the one-form $\kappa \in \Omega^{1}(M)$ iff the parabolic pair $\left(u, l^{\prime}\right)$ satisfies them with $\kappa$ replaced by:

$$
\kappa^{\prime}=\kappa+\mathrm{d} c-\lambda\left(c^{2} u+2 c l\right) .
$$

Similarly $(-u, l)$ satisfies them with $\kappa$ replaced by $-\kappa$. In particular, a nontrivial real Killing spinor on $(M, g)$ determines $\kappa$ up to transformations of the form:

$$
\kappa \rightarrow \zeta \kappa+\mathrm{d} c-\lambda\left(c^{2} u+2 c l\right), \text { with } \zeta \in\{-1,1\} \text { and } c \in \mathcal{C}^{\infty}(M) .
$$

Moreover, $(u, l)$ satisfies (69) for $\kappa$ iff $\left(u, l^{\prime}\right)$ satisfies them with $\kappa$ replaced by $\kappa^{\prime}$. Similarly, $(-u, l)$ satisfies (69) with $\kappa$ replaced by $-\kappa$.

By the Frobenius theorem, the first equation in (69) implies that the distribution $\operatorname{ker}(u)=$ $C_{u}^{\perp} \subset T M$ integrates to a codimension one foliation of $M$ which is transversally-orientable since $u$ is nowhere-vanishing. Since $C_{u}$ is contained in $C_{u}^{\perp}$, this foliation is degenerate in the sense that the restriction of $g$ to $C_{u}^{\perp}$ is a degenerate vector bundle metric. In particular, the three-dimensional vector space $C_{u, m}^{\perp}$ is tangent to the causal cone $\mathcal{L}_{m} \subset T_{m} M$ along the null line $C_{u, m}$ at any point $m \in M$ (see Appendix A) and the complement $C_{u}^{\perp} \backslash C_{u}$ consists of spacelike vectors. Since $l$ is orthogonal to $u$, we have $l^{\sharp} \in \Gamma\left(C_{u}^{\perp}\right)$. The vector fields $u^{\sharp}$ and $l^{\sharp}$ span a topologically trivial distribution $\Pi^{\sharp}$ of parabolic 2-planes contained in $C_{u}$.

Let $S\left(C_{u}^{\perp}\right)$ be any complement of $C_{u}$ in $C_{u}^{\perp}$ :

$$
C_{u}^{\perp}=C_{u} \oplus S\left(C_{u}^{\perp}\right) .
$$

Such a complement is known as a screen bundle of $C_{u}^{\perp}$ (see [24] and references therein); in our situation, it can be chosen such that $l^{\sharp} \in \Gamma\left(S\left(C_{u}^{\perp}\right)\right)$, in which case we can further decompose $S\left(C_{u}^{\perp}\right)=C_{l} \oplus L$, where $C_{l}$ is the rank one distribution spanned by $l^{\sharp}$ and $L$ is any rank one distribution complementary to $\Pi^{\sharp}$ in $C_{u}^{\perp}$. For any choice of the screen bundle, the restriction of $g$ to $S\left(C_{u}^{\perp}\right)$ is non-degenerate and positive-definite and hence admits an orthogonal complement in $T M$ which has the form $C_{u} \oplus C_{v}$, where $C_{v} \subset T M$ is the rank one distribution spanned by the unique null vector field $v^{\sharp} \in \mathfrak{X}(M)$ which is orthogonal to $S\left(C_{u}^{\perp}\right)$ and satisfies $g\left(u^{\sharp}, v^{\sharp}\right)=1$. This gives:

$$
T M=\left(C_{v} \oplus C_{u}\right) \oplus S\left(C_{u}^{\perp}\right),
$$

which allows us to write the metric as:

$$
g=u \otimes v+v \otimes u+q,
$$

where $q=\left.g\right|_{S\left(C_{u}^{\perp}\right)}$ and $v$ is the one-form dual to $v^{\sharp}$.

Lemma 5.10 Suppose that $(M, g)$ admits a nontrivial real Killing spinor $\epsilon \in \Gamma(S)$ with Killing constant $\frac{\lambda}{2} \neq 0$ and let $(u, l)$ be a corresponding parabolic pair of one-forms. Around every point in $M$, there exist local Walker-like coordinates $\left(x^{v}, x^{u}, x^{1}, x^{2}\right)$ with $u^{\sharp}=\partial_{x^{u}}$ in which the metric takes the form:

$$
d s_{g}^{2}=\mathcal{F}\left(d x^{v}\right)^{2}+2 \mathcal{K} d x^{v} d x^{u}+\omega_{i} d x^{v} d x^{i}+q_{i j} d x^{i} d x^{j},
$$

where $\mathcal{F}, \mathcal{K}, \omega_{i}$ and $q_{i j}$ are locally-defined functions which do not depend on $x^{u}$ and such that $\mathcal{K}$ is nowhere-vanishing. In these coordinates, the one-forms $u$ and $l$ can be written as:

$$
u=\mathcal{K} d x^{v}, \quad l=-\frac{1}{2 \lambda} d \log (\mathcal{K})+\mathfrak{s} d x^{v}=-\frac{1}{2 \lambda} d \log (\mathcal{K})+\frac{\mathfrak{s}}{\mathcal{K}} u,
$$

for some locally-defined function $\mathfrak{s}$. 
Proof Since $C_{u}^{\perp}$ is integrable and of corank one and $C_{u} \subset C_{u}^{\perp}$ has rank one, there exist local coordinates $\left(x^{v}, x^{u}, x^{1}, x^{2}\right)$ on $M$ such that $\partial_{x^{u}}=u^{\sharp}$, the vector fields $\partial_{x^{u}}, \partial_{x^{1}}, \partial_{x^{2}} \operatorname{span} C_{u}^{\perp}$ and $\partial_{x_{v}}$ is null. In such local coordinates, we have:

$$
u=g_{u v} \mathrm{~d} x^{v}, \quad \text { with } g_{u v}=u\left(\partial_{x^{v}}\right)=g\left(\partial_{x^{u}}, \partial_{x^{v}}\right) .
$$

Notice that $g_{u v}$ is nowhere-vanishing since $u$ is. The vector fields $\partial_{x^{1}}$ and $\partial_{x^{2}}$ span an integrable local screen $S\left(C_{u}^{\perp}\right)$ for $C_{u}^{\perp}$. Let $v$ be the unique null one form which vanishes along $S\left(C_{u}^{\perp}\right)$ and satisfies $v\left(\partial_{x^{u}}\right)=1$. Then the vector field $v^{\sharp}$ satisfies the assumptions which allow us to write the metric in the form (73). Writing $v=v_{v} \mathrm{~d} x^{v}+v_{u} \mathrm{~d} x^{u}+v_{i} \mathrm{~d} x^{i}$, the condition $v\left(\partial_{x^{u}}\right)=1$ implies $v_{u}=1$ and (73) gives:

$$
\begin{aligned}
g= & 2 g_{u v} v_{v} \mathrm{~d} x^{v} \otimes \mathrm{d} x^{v}+g_{u v}\left(\mathrm{~d} x^{v} \otimes \mathrm{d} x^{u}+\mathrm{d} x^{u} \otimes \mathrm{d} x^{v}\right) \\
& +g_{u v} v_{i}\left(\mathrm{~d} x^{v} \otimes \mathrm{d} x^{i}+\mathrm{d} x^{i} \otimes \mathrm{d} x^{v}\right)+q_{i j} \mathrm{~d} x^{i} \otimes \mathrm{d} x^{j} .
\end{aligned}
$$

Relabeling coefficients gives (74) with $\mathcal{F}=2 g_{u v} v_{v}, \mathcal{K}=g_{u v}$ and $\omega_{i}=2 g_{u v} v_{i}$. The coefficients of $g$ do not depend on $x^{u}$ since $u^{\sharp}=\partial_{x^{u}}$ is a Killing vector field. In these coordinates we have $u=\mathcal{K} \mathrm{d} x^{v}$ (hence $\mathcal{K}$ is nowhere-vanishing) and the first equation of the Pfaffian system (69) becomes:

$$
(\mathrm{d} \mathcal{K}+2 \lambda \mathcal{K} l) \wedge \mathrm{d} x^{v}=0,
$$

showing that:

$$
l=-\frac{1}{2 \lambda} \mathrm{d} \log (\mathcal{K})+\mathfrak{s} \mathrm{d} x^{v}
$$

for some locally-defined function $\mathfrak{s}$.

Lemma 5.10 gives existence of Walker-like coordinates on Lorentzian four-manifolds admitting real Killing spinors. These generalize the classical Walker coordinates [87] of Lorentzian manifolds which admit a parallel null line [17,37,87]. The main difference is that our $u$ is not recurrent. On the other hand, our $u$ is Killing - a condition which may not hold on generic Walker manifolds.

Example 5.11 The simply-connected four-dimensional anti-de Sitter space $\mathrm{AdS}_{4}$ admits Walker-like coordinates $\left(x^{v}, x^{u}, x, y\right)$ in which the Anti-de Sitter metric $g$ reads:

$$
\mathrm{d} s_{\mathrm{AdS}_{4}}^{2}=\frac{1}{c y^{2}}\left[\mathrm{~d} x^{v} \mathrm{~d} x^{u}+(\mathrm{d} x)^{2}+(\mathrm{d} y)^{2}\right],
$$

where $c$ is a positive constant equal to minus the curvature. It is well-known $[6,46,74]$ that $\mathrm{AdS}_{4}$ admits a four-dimensional space of real Killing spinors.

\subsection{The locally stationary and locally integrable case}

Definition 5.12 Suppose that $(M, g)$ admits a nontrivial real Killing spinor $\epsilon \in \Gamma(S)$ with nonzero Killing constant. We say that $(M, g, \epsilon)$ is:

- locally stationary if, around every point, the Walker-like coordinates induced by $\epsilon$ are such that $\partial_{x} v$ is Killing.

- locally integrable if, around every point, the Walker-like coordinates coordinates induced by $\epsilon$ are such that $\omega_{1}=\omega_{2}=0$. 
- locally static if, around every point, the Walker-like coordinates induced by $\epsilon$ are such that $\partial_{x^{v}}$ is Killing, $\omega_{1}=\omega_{2}=0$ and $\mathcal{F}=0$.

Remark 5.13 Notice that the locally defined rank two distribution $\Delta$ spanned by $\partial_{x^{v}}$ and $\partial_{x^{u}}$ is nondegenerate (and hence admits a non-vanishing timelike section) since $\partial_{x^{u}}$ is null and $\mathcal{K}=g\left(\partial_{x^{u}}, \partial_{x^{v}}\right)$ is locally nowhere-vanishing. If $(M, g, \epsilon)$ is locally stationary in the sense above then some linear combination of $\partial_{x^{v}}$ and $\partial_{x^{u}}$ is a timelike Killing vector field, whence $(M, g)$ is locally stationary in the standard sense. If $(M, g, \epsilon)$ is locally integrable, then the orthogonal complement of $\Delta$ is an integrable spacelike distribution of rank two. If $(M, g, \epsilon)$ is locally static then $\partial_{x^{v}}$ is null and $(M, g)$ admits a local hypersurface orthogonal to a time-like Killing vector field $X$ (namely $X=\partial_{x^{v}}+\partial_{x^{u}}$ or $X=\partial_{x^{v}}-\partial_{x^{u}}$ ), hence $(M, g)$ is locally static in the standard sense.

Theorem 5.14 The following statements are equivalent:

(a) There exists a nontrivial real Killing spinor $\epsilon \in \Gamma(S)$ with Killing constant $\frac{\lambda}{2} \neq 0$ on $(M, g)$ such that $(M, g, \epsilon)$ is locally stationary and locally integrable.

(b) $(M, g)$ is locally isometric to a Lorentzian four-manifold of the form:

$$
\left(\hat{M}, d s_{\hat{g}}^{2}\right)=\left(\mathbb{R}^{2} \times X, \mathcal{F}\left(d x^{v}\right)^{2}+2 \mathcal{K} d x^{v} d x^{u}+q\right),
$$

where $\left(x^{v}, x^{u}\right)$ are Cartesian coordinates on $\mathbb{R}^{2}, X$ is a non-compact, oriented and simply-connected surface endowed with the Riemannian metric $q$ and $\mathcal{F}, \mathcal{K} \in C^{\infty}(X)$ are functions on $X$ (with $\mathcal{K}$ nowhere-vanishing) which satisfy:

$$
\begin{aligned}
& \nabla^{q} d \mathcal{K}-\frac{d \mathcal{K} \otimes d \mathcal{K}}{2 \mathcal{K}}=2 \lambda^{2} \mathcal{K} q, \quad \Delta_{q} \mathcal{K}=6 \lambda^{2} \mathcal{K}, \quad \partial_{x^{u}} \mathfrak{s}=\kappa\left(\partial_{x^{u}}\right) \mathcal{K}, \\
& \frac{q^{*}(d \mathcal{K}, d \mathcal{F})}{4 \lambda \mathcal{K}}-\partial_{x^{v}} \mathfrak{s}=\lambda\left(\mathcal{F}-\mathfrak{s}^{2}\right)-\kappa\left(\partial_{x^{v}}\right) \mathcal{K}, \quad \partial_{x^{i}} \mathfrak{s}=\kappa\left(\partial_{x^{i}}\right) \mathcal{K}
\end{aligned}
$$

for some function $\mathfrak{s} \in C^{\infty}(M)$ and some one-form $\kappa \in \Omega^{1}(M)$, where $x^{1}, x^{2}$ are local coordinates on $X$.

In this case, the formulas:

$$
u=\mathcal{K} d x^{v}, \quad l=-\frac{1}{2 \lambda} d \log (\mathcal{K})+\mathfrak{s} d x^{v} .
$$

give a parabolic pair of one-forms $(u, l)$ corresponding to the real Killing spinor $\epsilon$, which satisfy Eq. (66) with respect to the one-form $\kappa$. Moreover, $(M, g)$ is Einstein with Einstein constant $\Lambda$ iff $\Lambda=-3 \lambda^{2}$ and the following equations are satisfied, where Ric ${ }^{q}$ is the Ricci tensor of $q$ :

$$
\Delta_{q} \mathcal{F}-\frac{q^{*}(d \mathcal{K}, d \mathcal{F})}{\mathcal{K}}=2 \lambda^{2} \mathcal{F}, \quad \operatorname{Ric}^{q}=-\lambda^{2} q,
$$

in which situation $(X, q)$ is a hyperbolic Riemann surface.

Remark 5.15 Here, the Laplacian $\Delta_{q}$ is defined through:

$$
\Delta_{q}(f) \stackrel{\text { def. }}{=} \operatorname{tr}\left(\nabla^{q} \operatorname{grad}_{q} f\right) \quad \forall f \in C^{\infty}(X) .
$$

The second equation in (75):

$$
\Delta_{q} \mathcal{K}=6 \lambda^{2} \mathcal{K}
$$


is a "wrong sign" eigenvalue problem for the Laplacian on $X$. Since $\Delta_{q}$ is negative semidefinite, no nontrivial solutions to (78) exists unless $X$ is non-compact. The function $\mathfrak{s}$ can be chosen at will as long as Eq. (75) hold, since different choices produce the same signed polyform square $\alpha=u+u \wedge l$ of $\epsilon$, namely:

$$
\alpha=\mathcal{K} \mathrm{d} x^{v}+\frac{1}{2 \lambda} \mathrm{d} \mathcal{K} \wedge \mathrm{d} x^{v}
$$

We can exploit this freedom to choose:

$$
\mathfrak{s}^{2}=\mathcal{F}-\frac{q^{*}(\mathrm{~d} \mathcal{K}, \mathrm{d} \mathcal{F})}{4 \lambda^{2} \mathcal{K}},
$$

which is independent of $x^{v}$ and $x^{u}$. For this choice of $\mathfrak{s}$, Eq. (75) reduce to:

$$
\kappa=\frac{\mathrm{d} \mathfrak{s}}{\mathcal{K}}, \quad \nabla^{q} \mathrm{~d} \mathcal{K}-\frac{\mathrm{d} \mathcal{K} \otimes \mathrm{d} \mathcal{K}}{2 \mathcal{K}}=2 \lambda^{2} \mathcal{K} q, \quad \Delta_{q} \mathcal{K}=6 \lambda^{2} \mathcal{K}
$$

and hence $\kappa$ is a one-form defined on $X$ which is completely determined by $\lambda, \mathcal{F}, \mathcal{K}$ and $q$. In particular, the condition that $(M, g)$ admits a nontrivial real Killing spinor with Killing constant $\frac{\lambda}{2}$ such that $(M, g, \epsilon)$ is locally stationary and locally integrable reduces to the last two equations in (81), which involve only $\lambda, \mathcal{K}$ and $q$ but do not involve $\mathcal{F}$. This generalizes a statement made in [46, page 391]. On the other hand, the Einstein condition (77) involves both $\mathcal{F}$ and $\mathcal{K}$. Strictly speaking, the "gauge choice" (80) requires:

$$
\mathcal{F}-\frac{q^{*}(\mathrm{~d} \mathcal{K}, \mathrm{d} \mathcal{F})}{4 \lambda^{2} \mathcal{K}} \geq 0
$$

if $l$ is to be well-defined, since the formula for $l$ involves $\mathfrak{s}$. However, the real Killing spinor associated to $(u, l)$ is well-defined and satisfies the Killing spinor equations even when $\mathfrak{s}^{2}$ is formally negative somewhere on $M$, because $\epsilon$ is determined by the polyform (79), which is independent of $\mathfrak{s}$.

Proof By Lemma 5.10 and Theorem 5.3, we must solve Eq. (66) for $u$ and $l$ of the form (76) , which automatically satisfy the first equation of the Pfaffian system (69). The first equation in (66) is equivalent to the condition that the vector field $u^{\sharp}=\partial_{x^{u}}$ is Killing, together with the condition the first equation in (69) holds. Thus it suffices to consider the second equation in (66). Evaluating this equation on $\partial_{x^{v}}$ and $\partial_{x^{u}}$ gives the system:

$$
\begin{aligned}
& \nabla_{\partial_{x} v}^{g} l=\kappa\left(\partial_{x^{v}}\right) u+\lambda l\left(\partial_{x^{v}}\right) l-\lambda g\left(\partial_{x^{v}}\right) \\
& \nabla_{\partial_{x} u}^{g} l=\kappa\left(\partial_{x^{u}}\right) u+\lambda l\left(\partial_{x^{u}}\right) l-\lambda g\left(\partial_{x^{u}}\right),
\end{aligned}
$$

which reduce to the following equations for $u$ and $l$ as in (76):

$$
\begin{aligned}
& \frac{q^{*}(\mathrm{~d} \mathcal{K}, \mathrm{d} \mathcal{F})}{4 \lambda \mathcal{K}}-\partial_{x^{v}} \mathfrak{s}=\lambda \mathcal{F}-\lambda \mathfrak{s}^{2}-\kappa\left(\partial_{x^{v}}\right) \mathcal{K}, \\
& \frac{q^{*}(\mathrm{~d} \mathcal{K}, \mathrm{d} \mathcal{K})}{4 \lambda \mathcal{K}}=\lambda \mathcal{K}, \quad \partial_{x^{u}} \mathfrak{s}=\kappa\left(\partial_{x^{u}}\right) \mathcal{K} .
\end{aligned}
$$

On the other hand, restricting the second equation in (66) to $X$ and using (76) gives:

$$
\nabla^{q} \mathrm{~d} \mathcal{K}-\frac{\mathrm{d} \mathcal{K} \otimes \mathrm{d} \mathcal{K}}{2 \mathcal{K}}=2 \lambda^{2} \mathcal{K} q, \quad \partial_{x^{i}} \mathfrak{s}=\kappa\left(\partial_{x^{i}}\right) \mathcal{K} .
$$

Furthermore, taking the trace of (83) and combining it with the third equation in (82) we obtain $\Delta_{q} \mathcal{K}=6 \lambda^{2} \mathcal{K}$. Together with relations (82) and (83), this establishes the system (75). 
Consider now the Einstein equation $\operatorname{Ric}^{g}=\Lambda g$ on $(M, g)$. The only non-trivial components are:

$$
\operatorname{Ric}^{g}\left(\partial_{x^{v}}, \partial_{x^{v}}\right)=\Lambda \mathcal{F}, \quad \operatorname{Ric}^{g}\left(\partial_{x^{u}}, \partial_{x^{v}}\right)=\Lambda \mathcal{K},\left.\quad \operatorname{Ric}^{g}\right|_{T X}=\Lambda q .
$$

Direct computation gives:

$$
\begin{aligned}
& \operatorname{Ric}^{g}\left(\partial_{x^{v}}, \partial_{x^{v}}\right)=-\frac{1}{2} \Delta_{q} \mathcal{F}+\frac{1}{2 \mathcal{K}} q^{*}(\mathrm{~d} \mathcal{K}, \mathrm{d} \mathcal{F})-\frac{\mathcal{F}}{2 \mathcal{K}^{2}} q^{*}(\mathrm{~d} \mathcal{K}, \mathrm{d} \mathcal{K}), \\
& \operatorname{Ric}^{g}\left(\partial_{x^{v}}, \partial_{x^{u}}\right)=-\frac{1}{2} \Delta_{q} \mathcal{K},\left.\quad \operatorname{Ric}^{g}\right|_{T X}=\operatorname{Ric}^{q}-\frac{\nabla^{q} \mathrm{~d} \mathcal{K}}{\mathrm{d} \mathcal{K}}+\frac{\mathrm{d} \mathcal{K} \otimes \mathrm{d} \mathcal{K}}{2 \mathcal{K}^{2}} .
\end{aligned}
$$

Combining these relations with (75), we conclude.

Remark 5.16 Lorentzian four-manifolds admitting nontrivial real Killing spinors are supersymmetric configurations of four-dimensional $\mathcal{N}=1$ minimal AdS supergravity [28,81]. Supersymmetric solutions of that theory are Lorentzian four-manifolds admitting nontrivial real Killing spinors which satisfy the Einstein equation with negative cosmological constant. Hence Theorem 5.14 characterizes all locally integrable and locally stationary supersymmetric solutions of this theory. To our best knowledge, the classification of four-dimensional Lorentzian manifolds admitting real Killing spinors is currently open. Theorem 5.3 and [37] could be used to attack this problem in full generality.

Theorem 5.14 suggests a strategy to construct Lorentzian four-manifolds admitting real Killing spinors. Fix a simply-connected (generally incomplete) hyperbolic Riemann surface $(X, q)$ and consider the eigenspace of the Laplacian on $(X, q)$ with eigenvalue $6 \lambda^{2}$. In this space, look for a function $\mathcal{K}$ which satisfies the first equation in (75). If such exists, it gives a real Killing spinor for any $\mathcal{F} \in C^{\infty}(X)$. Below, we give special classes of solutions when $(X, q)$ is the Poincaré half-plane.

\subsection{Special solutions from the Poincaré half-plane}

Take:

$$
\left(M, \mathrm{~d} s_{g}^{2}\right)=\left(\mathbb{R}^{2} \times \mathbb{H}, \mathcal{F}\left(\mathrm{d} x^{v}\right)^{2}+2 \mathcal{K} \mathrm{d} x^{v} \mathrm{~d} x^{u}+c \frac{(\mathrm{d} x)^{2}+(\mathrm{d} y)^{2}}{y^{2}}\right),
$$

where $x, y$ are global coordinates on the Poincaré half-plane $\mathbb{H}=\left\{(x, y) \in \mathbb{R}^{2} \mid y>0\right\}$ and $\mathcal{F}, \mathcal{K}$ are real-valued functions defined on $\mathbb{H}$ (with $\mathcal{K}$ nowhere-vanishing), while $c>0$ is a constant. Let $q$ denote the metric $c \frac{\mathrm{d} x \otimes \mathrm{d} x+\mathrm{d} y \otimes \mathrm{d} y}{y^{2}}$ on $\mathbb{H}$. Theorem 5.14 shows that such $(M, g)$ admits a real Killing spinor with Killing constant $\frac{\lambda}{2} \neq 0$ iff:

$$
\nabla^{q} \mathrm{~d} \mathcal{K}-\frac{\mathrm{d} \mathcal{K} \otimes \mathrm{d} \mathcal{K}}{2 \mathcal{K}}=2 \lambda^{2} \mathcal{K} q, \quad \Delta_{q} \mathcal{K}=6 \lambda^{2} \mathcal{K},
$$

in which case $(M, g)$ is Einstein iff:

$$
\Delta_{q} \mathcal{F}-\frac{q^{*}(\mathrm{~d} \mathcal{K}, \mathrm{d} \mathcal{F})}{\mathcal{K}}=2 \lambda^{2} \mathcal{F}
$$

Direct computation shows that Eq. (85) are equivalent with:

$$
\begin{aligned}
& \partial_{x}^{2} \mathcal{K}-\frac{\partial_{y} \mathcal{K}}{y}=\frac{\left(\partial_{x} \mathcal{K}\right)^{2}}{2 \mathcal{K}}+\frac{2 \lambda^{2} c}{y^{2}} \mathcal{K}, \quad \partial_{y}^{2} \mathcal{K}+\frac{\partial_{y} \mathcal{K}}{y}=\frac{\left(\partial_{y} \mathcal{K}\right)^{2}}{2 \mathcal{K}}+\frac{2 \lambda^{2} c}{y^{2}} \mathcal{K} \\
& \partial_{x y}^{2} \mathcal{K}+\frac{\partial_{x} \mathcal{K}}{y}=\frac{\partial_{x} \mathcal{K} \partial_{y} \mathcal{K}}{2 \mathcal{K}}, \quad y^{2}\left(\partial_{x}^{2} \mathcal{K}+\partial_{y}^{2} \mathcal{K}\right)=6 \lambda^{2} c \mathcal{K}
\end{aligned}
$$


This gives the following:

Corollary 5.17 The Lorentzian four-manifold (84) admits a nontrivial real Killing spinor with Killing constant $\frac{\lambda}{2} \neq 0$ iff:

$$
\begin{aligned}
& \partial_{y}^{2} \mathcal{K}+\frac{\partial_{y} \mathcal{K}}{y}=\frac{\left(\partial_{y} \mathcal{K}\right)^{2}}{2 \mathcal{K}}+\frac{2 \lambda^{2} c}{y^{2}} \mathcal{K}, \quad \partial_{x y}^{2} \mathcal{K}+\frac{\partial_{x} \mathcal{K}}{y}=\frac{\partial_{x} \mathcal{K} \partial_{y} \mathcal{K}}{2 \mathcal{K}} \\
& \left(\partial_{x} \mathcal{K}\right)^{2}+\left(\partial_{y} \mathcal{K}\right)^{2}=\frac{4 \lambda^{2} c}{y^{2}} \mathcal{K}^{2}, \quad y^{2}\left(\partial_{x}^{2} \mathcal{K}+\partial_{y}^{2} \mathcal{K}\right)=6 \lambda^{2} . c \mathcal{K}
\end{aligned}
$$

In this case, it is Einstein iff $\mathcal{F}$ satisfies (86).

Choosing $\mathcal{F}$ to not satisfy (86) produces large families of non-Einstein Lorentzian fourmanifolds admitting real Killing spinors.

Example 5.18 Taking $\mathcal{K}=\mathcal{F}=\frac{c}{y^{2}}$ gives a solution of (87) iff $c \lambda^{2}=1$. Hence the Lorentzian four-manifold:

$$
\left(M, \mathrm{~d} s_{g}^{2}\right)=\left(\mathbb{R}^{2} \times \mathbb{H}, \frac{1}{\lambda^{2} y^{2}}\left[\left(\mathrm{~d} x^{v}\right)^{2}+2 \mathrm{~d} x^{v} \mathrm{~d} x^{u}+(\mathrm{d} x)^{2}+(\mathrm{d} y)^{2}\right]\right)
$$

admits a real Killing spinor. This is the $\mathrm{AdS}_{4}$ space with metric written in horospheric coordinates [44], which is well-known to admit the maximal number (namely four) of real Killing spinors [11].

More examples can be constructed by solving in more generality the eigenvector problem for the Laplace operator of the Poincaré half plane and checking which solutions satisfy the first equation in (85). We illustrate this by constructing solutions obtained through separation of variables. Set:

$$
\mathcal{K}=k_{x} k_{y},
$$

where $k_{x} \in \mathcal{C}^{\infty}(\mathbb{H})$ depends only on $x$ and $k_{y} \in \mathcal{C}^{\infty}(\mathbb{H})$ depends only on $y$. The second equation in (87) gives:

$$
\dot{k}_{x}\left(\dot{k}_{y}+\frac{2 k_{y}}{y}\right)=0,
$$

where the dot denotes derivation with respect to the corresponding variable. When $\dot{k}_{x}=0$, Eq. (87) reduce to:

$$
\partial_{y} \mathcal{K}+\frac{2}{y} \mathcal{K}=0, \quad \lambda^{2} c=1,
$$

with general solution $\mathcal{K}=c_{0} y^{-2}$ (where $c_{0} \neq 0$ is a constant). If $\dot{k}_{x} \neq 0$, then (88) gives $\dot{k}_{y}+\frac{2 k_{y}}{y}=0$, so $k_{y}=c_{0} y^{-2}$ for a non-zero constant $c_{0}$. Using this in (87) gives $\dot{k}_{x}=0$, a contradiction. Hence every Lorentzian four-manifold of the form:

$$
\left(M, \mathrm{~d} s_{g}^{2}\right)=\left(\mathbb{R}^{2} \times \mathbb{H}, \mathcal{F}\left(\mathrm{d} x^{v}\right)^{2}+2 c_{0} \frac{\mathrm{d} x^{v} \mathrm{~d} x^{u}}{y^{2}}+\frac{(\mathrm{d} x)^{2}+(\mathrm{d} y)^{2}}{\lambda^{2} y^{2}}\right)
$$

with $\mathcal{F}$ a smooth function admits nontrivial real Killing spinors. This gives large families of non-Einstein Lorentzian four-manifolds carrying real Killing spinors by taking $\mathcal{F}$ to be 
generic. The Lorentzian manifold (89) is Einstein when Eq. (86) is satisfied, which for $\mathcal{K}=c_{0} y^{-2}$ reads:

$$
y^{2}\left(\partial_{x}^{2} \mathcal{F}+\partial_{y}^{2} \mathcal{F}\right)+2 y \partial_{y} \mathcal{F}=2 \mathcal{F} .
$$

To study (90), we try the separated Ansatz:

$$
\mathcal{F}=f_{x} f_{y},
$$

where $f_{x} \in \mathcal{C}^{\infty}(\mathbb{H})$ depends only on $x$ and $f_{y} \in \mathcal{C}^{\infty}(\mathbb{H})$ depends only on $y$. Equation (90) gives:

$$
\frac{\ddot{f}_{x}}{f_{x}}=c^{2}=\frac{\ddot{f}_{y}}{f_{y}}+\frac{2 \dot{f}_{y}}{y f_{y}}-\frac{2}{y^{2}}
$$

for some $c \in \mathbb{R}$. If $c=0$, this is solved by:

$$
f_{x}=a_{1}+a_{2} x, \quad f_{y}=a_{3} y+\frac{a_{4}}{y^{2}},
$$

where $\mathbf{a} \stackrel{\text { def. }}{=}\left(a_{1}, \ldots, a_{4}\right) \in \mathbb{R}^{4}$. This gives the following family of Einstein Lorentzian metrics on $\mathbb{R}^{2} \times \mathbb{H}$ admitting real Killing spinors, where we eliminated $c_{0}$ by rescaling $x^{u}$ :

$$
\mathrm{d} s^{g}=\left(a_{1}+a_{2} x\right)\left(a_{3} y+\frac{a_{4}}{y^{2}}\right)\left(\mathrm{d} x^{v}\right)^{2}+\frac{\mathrm{d} x^{v} \mathrm{~d} x^{u}}{y^{2}}+\frac{(\mathrm{d} x)^{2}+(\mathrm{d} y)^{2}}{\lambda^{2} y^{2}} .
$$

For $a_{1}=a_{2}=a_{3}=a_{4}=0$ we recover the $\mathrm{AdS}_{4}$ metric written in horospheric coordinates. Thus (92) gives a four-parameter deformation of $\mathrm{AdS}_{4}$. Every choice $\mathbf{a} \in \mathbb{R}^{4}$ produces an Einstein metric on $\mathbb{R}^{2} \times \mathbb{H}$ with Einstein constant $\Lambda=-3 \lambda^{2}$ admitting real Killing spinors.

If $c \neq 0$, the first equation in (91) gives:

$$
f_{x}=a_{1} e^{c x}+a_{2} e^{-c x}
$$

with $a_{1}, a_{2} \in \mathbb{R}$. On the other hand, the equation for $f_{y}$ can be written as:

$$
y^{2} \ddot{f}_{y}+2 y \dot{f}_{y}+\left(c^{2} y^{2}-2\right) f_{y}=0,
$$

being the radial part of the Helmholtz equation in spherical coordinates. Its general solution is a linear combination of the spherical Bessel functions BY and BJ:

$$
f_{y}=a_{3} \mathrm{BY}(c y)+a_{4} \mathrm{BJ}(c y),
$$

where $a_{3}, a_{4} \in \mathbb{R}$. We have:

$$
\mathrm{BJ}(c y)=\frac{\sin (c y)}{c^{2} y^{2}}-\frac{\cos (c y)}{c y}, \mathrm{BY}(c y)=-\frac{\cos (c y)}{c^{2} y^{2}}-\frac{\sin (c y)}{c y},
$$

whence:

$$
\mathrm{d} s_{g}^{2}=\left(a_{1} e^{c x}+a_{2} e^{-c x}\right)\left[a_{3} \mathrm{BY}(c y)+a_{4} \mathrm{BJ}(c y)\right]\left(\mathrm{d} x^{v}\right)^{2}+\frac{\mathrm{d} x^{v} \mathrm{~d} x^{u}}{y^{2}}+\frac{(\mathrm{d} x)^{2}+(\mathrm{d} y)^{2}}{\lambda^{2} y^{2}} .
$$

This gives a four-parameter family (parameterized by $\left.\left(a_{1}, a_{2}, a_{3}, a_{4}\right) \in \mathbb{R}^{4}\right)$ of Lorentzian Einstein metrics on $\mathbb{R}^{2} \times \mathbb{H}$ admitting real Killing spinors.

Remark 5.19 When $a_{1} a_{2} \neq 0$ and $a_{3} \neq 0$, the Lorentzian four-manifolds constructed above are not isometric to $\mathrm{AdS}_{4}$, since their Weyl tensor is non-zero and their Riemann tensor is not parallel. 


\section{Supersymmetric heterotic configurations}

In this section we consider generalized constrained Killing spinors in an abstract form of heterotic supergravity (inspired by [39]), which is parameterized by a triplet $(M, P, \mathfrak{c})$, where $M$ is a spin open four-manifold, $P$ is a principal bundle over $M$ with compact semi-simple Lie structure group $\mathrm{G}$ and $\mathfrak{c}$ is an $\mathrm{Ad}_{G}$-invariant, symmetric and non-degenerate inner product on the Lie algebra $\mathfrak{g}$ of $G$. Let $\mathfrak{g}_{P} \stackrel{\text { def. }}{=} P \times \operatorname{Ad}_{G} \mathfrak{g}$ be the adjoint bundle of $P$. The Killing spinor equations of heterotic supergravity couple a strongly spinnable Lorentzian metric $g$ on $M$ (taken to be of "mostly plus" signature), a closed one-form $\varphi \in \Omega^{1}(M)$, a three-form $H \in$ $\Omega^{3}(M)$ and a connection $A$ on $P$. This system of partial differential equations characterizes supersymmetric configurations of the theory defined by $(M, P, \mathfrak{c})$. For simplicity, we assume $H^{1}\left(M, \mathbb{Z}_{2}\right)=0$, although this assumption can be relaxed. We refer the reader to Appendix 6 for certain details.

\subsection{Supersymmetric heterotic configurations}

Let us fix a triple $(M, P, \mathfrak{c})$ as above, where $H^{1}\left(M, \mathbb{Z}_{2}\right)=0$. For every strongly-spinnable metric $g$ of signature $(3,1)$ on $M$, let $\mathbf{S}_{g}=(S, \Gamma, \mathscr{B})$ be a paired real spinor bundle on $(M, g)$, where the admissible pairing $\mathscr{B}$ is skew-symmetric and of negative adjoint type. With our assumptions, $\mathbf{S}_{g}$ is unique up to isomorphism of paired spinor bundles, combined with a rescaling of $\mathscr{B}$ by a non-zero constant. Let $F_{A} \in \Omega^{2}\left(\mathfrak{g}_{P}\right)$ denote the curvature form of a connection $A$ on $P$. As explained in Appendix 6, $g$ and $\mathfrak{c}$ induce a symmetric morphism of vector bundles $\mathfrak{c}(-\wedge-): \Lambda\left(M, \mathfrak{g}_{p}\right) \otimes \Lambda\left(M, \mathfrak{g}_{p}\right) \rightarrow \Lambda(M)$, where $\Lambda\left(M, \mathfrak{g}_{p}\right) \stackrel{\text { def. }}{=}$ $\Lambda(M) \otimes \mathfrak{g}_{P}$. For any 3-form $H \in \Omega^{3}(M)$, let $\hat{\nabla}^{H}$ be the natural lift to $S_{g}$ of the unique metric connection on $(M, g)$ with totally skew-symmetric torsion given by $-H$.

Definition 6.1 A heterotic configuration for $(M, P, \mathfrak{c})$ is an ordered quadruplet $(g, \varphi, H, A)$, where $g$ is a strongly-spinnable Lorentzian metric on $M, \varphi \in \Omega^{1}(M)$ is a closed one-form, $H \in \Omega^{3}(M)$ is a three-form and $A \in \mathcal{A}_{P}$ is a connection on $P$ such that the modified Bianchi identity holds:

$$
\mathrm{d} H=\mathfrak{c}\left(F_{A} \wedge F_{A}\right) .
$$

The configuration is called supersymmetric if there exists a nontrivial spinor $\epsilon \in \Gamma\left(S_{g}\right)$ such that:

$$
\hat{\nabla}^{H} \epsilon=0, \varphi \cdot \epsilon=H \cdot \varepsilon, \quad F_{A} \cdot \epsilon=0 .
$$

Remark 6.2 Equations (94) encode vanishing of the gravitino, dilatino and gaugino supersymmetry variations. Since we work in Lorentzian signature, supersymmetric configurations need not solve the equations of motion (which are given in Appendix 6). However, the study of supersymmetric configurations is a first step toward classifying supersymmetric solutions. The study of supersymmetric solutions this theory in the physical case of ten Lorentzian dimensions was pioneered in [48-50], where their local structure was characterized. The last equation in (94) is formally identical to the spinorial characterization of instantons in Riemannian signature and dimensions from four to eight. 


\subsection{Characterizing supersymmetric heterotic configurations through differential forms}

The metric connection $\nabla^{H}$ is given by (see Appendix 6 for notation):

$$
\nabla_{Y}^{H} X=\nabla_{Y}^{g} X-\frac{1}{2} H^{\sharp}(X, Y) \quad \forall X, Y \in \mathfrak{X}(M),
$$

where $\nabla^{g}$ is the Levi-Civita connection of $(M, g)$ and $H^{\sharp}$ is $H$ viewed as a $T M$-valued two-form. Hence the first equation in (94) can be written as:

$$
\nabla_{X}^{g} \epsilon-\frac{1}{4} H(X) \cdot \epsilon=0 \quad \forall X \in \mathfrak{X}(M),
$$

where $H(X) \stackrel{\text { def. }}{=} \iota_{X} H \in \Omega^{2}(M)$. This shows that $\epsilon$ is a generalized Killing spinor relative to the connection $\mathcal{D}=\nabla^{S}-\mathcal{A}$ on $S$, where:

$$
\hat{\mathcal{A}}_{X} \stackrel{\text { def. }}{=} \Psi_{\Gamma}^{-1}(\mathcal{A})=\frac{1}{4} H(X)=\frac{1}{4}(* \rho)(X)=\frac{1}{4} *\left(\rho \wedge X^{\mathrm{b}}\right) \quad \forall X \in \mathfrak{X}(M)
$$

and we defined $\rho \stackrel{\text { def. }}{=} * H \in \Omega^{1}(M)$. The second and third conditions in (94) are linear algebraic constraints on $\epsilon$. Hence the first three equations of (94) state that $\epsilon$ is a constrained generalized Killing spinor (see Definition 4.15). As in Sects. 3.6.3 and 4.6, consider a signed polyform square of $\epsilon$ :

$$
\alpha=u+u \wedge l,
$$

where $(u, l)$ is a parabolic pairs of one-forms.

Lemma $6.3(g, \varphi, H, A, \epsilon)$ satisfies the second equation in (94) (the dilatino equation) iff:

$$
\begin{aligned}
& \varphi \wedge u=-*(\rho \wedge u), \varphi \wedge u \wedge l=g^{*}(\rho, l) * u, \quad g^{*}(\varphi, l) u=*(l \wedge u \wedge \rho), \\
& g^{*}(u, \varphi)=0, \quad g^{*}(u, \rho)=0, \quad g^{*}(\rho, \varphi)=0,
\end{aligned}
$$

where $\rho \stackrel{\text { def. }}{=} * H$.

Proof By Proposition 3.26, the dilatino equation holds iff:

$$
\varphi \diamond \alpha=H \diamond \alpha
$$

where $\alpha=u+u \wedge l$ is a signed polyform square of $\epsilon$. We compute:

$$
\begin{aligned}
\varphi \diamond \alpha & =g^{*}(\varphi, u)+\varphi \wedge u+\varphi \wedge u \wedge l+g^{*}(\varphi, u) l-g^{*}(\varphi, l) u, \\
H \diamond \alpha & =v \diamond \rho \diamond \alpha=g^{*}(\rho, u) v-*(\rho \wedge u)-*(l \wedge u \wedge \rho)+g^{*}(\rho, u) * l+g^{*}(\rho, l) * u,
\end{aligned}
$$

where in the second equation we used (37) to rewrite the geometric product in terms of $\rho$. Separating degrees in (95) and using these relations gives the conclusion.

Lemma $6.4(g, \varphi, H, A, \epsilon)$ satisfies the third equation in (94) (the gaugino equation) iff:

$$
F_{A}=u \wedge \chi_{A},
$$

where $\chi_{A} \in \Gamma\left(u^{\perp} \otimes \mathfrak{g}_{P}\right)$ is a $\mathfrak{g}_{P}$-valued one-form orthogonal to $u$. 
Proof By Proposition 3.26, the gaugino equation holds iff:

$$
\alpha \diamond F_{A}=u \diamond F_{A}+u \diamond l \diamond F_{A}=0 .
$$

Expanding the geometric product gives:

$$
\alpha \diamond F_{A}=u \wedge F_{A}+\iota_{u} F_{A}+u \wedge l \wedge F_{A}-l \wedge \iota_{u} F_{A}+u \wedge \iota_{l} F_{A}+\iota_{u} \iota_{l} F_{A}=0 .
$$

Hence separating degrees in (97) gives the system:

$$
u \wedge F_{A}=0, \quad \iota_{u} F_{A}=0,
$$

which is solved by (96).

Lemma $6.5(g, \varphi, H, A, \epsilon)$ satisfies the first equation in (94) (the gravitino equation) iff:

$$
\nabla^{g} u=\frac{1}{2} *(\rho \wedge u), \quad \nabla^{g} l=\frac{1}{2} *(\rho \wedge l)+\kappa \otimes u .
$$

where $\kappa \in \Omega^{1}(M)$ and $\rho \stackrel{\text { def. }}{=} * H$. In this case, $u^{\sharp} \in \mathfrak{X}(M)$ is a Killing vector field.

Proof By Theorem 4.26, the gravitino equation holds iff:

$$
\nabla_{v}^{g}(u+u \wedge l)=\frac{1}{4}[H(v) \diamond(u+u \wedge l)-(u+u \wedge l) \diamond H(v)] \quad \forall v \in \mathfrak{X}(M),
$$

which reduces to the following upon expanding the geometric product and separating degrees:

$$
2 \nabla_{v}^{g} u+H(v, u)=0,2 \nabla_{v}^{g}(u \wedge l)+H(v, u) \wedge l+u \wedge H(v, l)=0 .
$$

This system is equivalent with:

$$
2 \nabla_{v}^{g} u+H(v, u)=0, \quad 2 \nabla_{v}^{g} l+H(v, l)-2 \kappa(v) u=0 \quad \forall v \in \mathfrak{X}(M)
$$

for some one-form $\kappa \in \Omega^{1}(M)$. In turn, this is equivalent with (98).

Theorem 6.6 A quadruplet $(g, \varphi, H, A)$ is a supersymmetric heterotic configuration for $(M, P, \mathfrak{c})$ iff there exists a parabolic pair of one-forms $(u, l)$ such that the following equations (where $\rho \stackrel{\text { def. }}{=} * H \in \Omega^{1}(M)$ ) are satisfied:

$$
\begin{aligned}
& \varphi \wedge u=*(\rho \wedge u), \varphi \wedge u \wedge l=-g^{*}(\rho, l) * u, \quad-g^{*}(\varphi, l) u=*(l \wedge u \wedge \rho), \\
& g^{*}(u, \varphi)=0, g^{*}(u, \rho)=0, \quad g^{*}(\rho, \varphi)=0, \quad F_{A}=u \wedge \chi_{A}, \\
& \nabla^{g} u=\frac{1}{2} u \wedge \varphi, \quad \nabla^{g} l=\frac{1}{2} *(\rho \wedge l)+\kappa \otimes u, \quad d^{*} \rho=0,
\end{aligned}
$$

for some one-form $\kappa \in \Omega^{1}(M)$ and some $\mathfrak{g}_{P}$-valued one-form $\chi_{A} \in \Omega^{1}\left(M, \mathfrak{g}_{P}\right)$ which is orthogonal to $u$. In this case, $u^{\sharp} \in \mathfrak{X}(M)$ is a Killing vector field and the distribution ker $u \subset T M$ integrates to a transversely-orientable codimension one foliation of $M$.

Proof By Lemmas 6.3, 6.4 and 6.5, it suffices to prove the last equation in the third line of (99). The modified Bianchi identity can be written as:

$$
\mathrm{d} * \rho=*^{2} \mathfrak{c}\left(F_{A} \wedge F_{A}\right),
$$

and gives:

$$
\mathrm{d}^{*} \rho=\mathfrak{c}\left(\iota_{u} \chi_{A}, \iota_{u} \chi_{A}\right)=\mathfrak{c}\left(\chi_{A}(u), \chi_{A}(u)\right)=0
$$


because $\chi_{A}$ is orthogonal to $u$. The first equation in the the third line of (99) amounts to:

$$
\mathcal{L}_{u^{\sharp}} g=0, \mathrm{~d} u=\frac{1}{8} u \wedge \varphi,
$$

showing that $u^{\sharp}$ is Killing and the distribution ker $u \subset T M$ is integrable, giving a transverselyorientable foliation $\mathcal{F}_{u} \subset M$ of codimension one.

\subsection{Some examples}

Example 6.7 Let:

$$
\left(M, \mathrm{~d} s_{g}^{2}\right)=\left(\mathbb{R}^{2} \times X, 2 \mathrm{~d} x^{v} \mathrm{~d} x^{u}+q\left(x^{v}\right)\right),
$$

where $q\left(x^{v}\right)$ is a flat Riemannian metric on $X$ for all $x^{v} \in \mathbb{R}$ and take $P$ to be the unit principal bundle over $M$. Consider the spinor $\epsilon$ corresponding to the pair $(u, l)$, where $u=\mathrm{d} x^{v}$ and $l=l\left(x^{v}\right)$ depends on $x^{v}$. Finally, take:

$$
\varphi=\Omega u, \quad \rho=0,
$$

where $\Omega \in C^{\infty}\left(\mathbb{R}^{2} \times X\right)$. A short computation shows that Eq. (99) reduce to:

$$
\nabla^{g} l=\kappa \otimes \mathrm{d} x^{v} .
$$

Applying this to $\partial_{x^{v}}, \partial_{x^{u}}$ and restricting to $T X$ gives:

$$
\begin{aligned}
& \left.\nabla_{x^{v}}^{g} l=\partial_{x^{v}} l-\frac{1}{2} l^{\sharp}\right\lrcorner \partial_{x^{v}} q\left(x^{v}\right)=0, \quad \kappa\left(\partial_{x^{v}}\right)=0, \quad \kappa\left(\partial_{x^{u}}\right)=0, \\
& \left.\left.\nabla^{g} l\right|_{T^{*} X}=\nabla^{q} l-\frac{1}{2} l^{\sharp}\right\lrcorner \partial_{x^{v}} q\left(x^{v}\right) \mathrm{d} x^{v}=\left(\kappa_{X}-*_{q\left(x^{v}\right)} l\right) \otimes \mathrm{d} x^{v},
\end{aligned}
$$

where $\left.\kappa_{X} \stackrel{\text { def. }}{=} \kappa\right|_{T X}$. This implies:

$$
\left.\nabla^{q} l=0, \quad \kappa_{X}=*_{q\left(x^{v}\right)} l-\frac{1}{2} l^{\sharp}\right\lrcorner \partial_{x^{v}} q\left(x^{v}\right),
$$

showing that $\left(X, q\left(x^{v}\right)\right)$ is flat for all $x^{v} \in \mathbb{R}$. The only remaining non-trivial condition is:

$$
\left.\partial_{x^{v}} l=\frac{1}{2} l^{\sharp}\right\lrcorner \partial_{x^{v}} q\left(x^{v}\right) .
$$

This is a linear first order ordinary differential equation for the function $x^{v} \rightarrow l\left(x^{v}\right)$. For every choice of parallel vector field on $\left(X, q\left(x_{0}^{v}\right)\right)$ with fixed $\left(x_{0}^{v}, x_{0}^{u}\right) \in \mathbb{R}^{2}$, its solution with the corresponding initial condition determines a one-parameter family of one forms $\left\{l\left(x^{v}\right)\right\}_{x^{v} \in \mathbb{R}}$ on $\left(X, q\left(x^{v}\right)\right)$. Assuming for instance that $X$ is simply connected and that $q\left(x^{v}\right)$ satisfies:

$$
\partial_{x^{v}} q\left(x^{v}\right)=2 F\left(x^{v}\right) q\left(x^{v}\right),
$$

for some function $F\left(x^{v}\right)$ depending only on $x^{v}$, then the explicit solution is:

$$
l\left(x^{v}\right)=e^{\int F\left(x^{v}\right)} l_{0}
$$

where $l_{0}$ is parallel vector field on $\left(X, q\left(x_{0}^{v}\right)\right)$ and we canonically identify the tangent vector spaces of $\left\{\left(x^{v}, x^{u}\right)\right\} \times X$ at different points $\left(x^{v}, x^{u}\right)$. 
Remark 6.8 Heterotic solutions with exact null dilaton were considered before (see [13]). As remarked earlier, a supersymmetric heterotic configuration need not be a solution of the equation of motion given in Appendix B. The classification of (geodesically complete) supersymmetric heterotic solutions on a Lorentzian four-manifold and the diffeomorphism type of four-manifolds admitting such solutions for fixed principal bundle topology is an open problem. Appendix B gives a brief formulation of abstract bosonic heterotic supergravity and its Killing spinor equations.

Acknowledgements The work of C. I. L. was supported by Grant IBS-R003-S1. The work of C. S. S. is supported by the Humboldt Research Fellowship ESP 1186058 HFST-P from the Alexander von Humboldt Foundation. The research of V.C. and C.S.S. was partially funded by the Deutsche Forschungsgemeinschaft (DFG, German Research Foundation) under Germany's Excellence Strategy_EXC 2121 Quantum Universe390833306

Funding Open Access funding enabled and organized by Projekt DEAL.

Open Access This article is licensed under a Creative Commons Attribution 4.0 International License, which permits use, sharing, adaptation, distribution and reproduction in any medium or format, as long as you give appropriate credit to the original author(s) and the source, provide a link to the Creative Commons licence, and indicate if changes were made. The images or other third party material in this article are included in the article's Creative Commons licence, unless indicated otherwise in a credit line to the material. If material is not included in the article's Creative Commons licence and your intended use is not permitted by statutory regulation or exceeds the permitted use, you will need to obtain permission directly from the copyright holder. To view a copy of this licence, visit http://creativecommons.org/licenses/by/4.0/.

\section{Appendix A: Parabolic 2-planes and degenerate complete flags in $\mathbb{R}^{3,1}$}

Let $(V, h)$ be a four-dimensional Minkowski space of "mostly plus" signature. A non-zero subspace $W \subset V^{*}$ is called degenerate or nondegenerate according to whether the restriction $h_{W}^{*}$ of $h^{*}$ to $W$ is a degenerate or non-degenerate quadratic form. A non-degenerate subspace $W$ is called:

- positive or negative definite, if the restriction $h_{W}^{*}$ to $W$ is positive or negative negative definite, respectively

- hyperbolic, if the restriction of $h^{*}$ to $W$ is not positive or negative definite.

Notice that $W$ is partially isotropic (i.e. contains nonzero null vectors) iff it is degenerate or hyperbolic. Let $\mathcal{L}$ denote the cone of causal (i.e. non-spacelike) vectors in $\left(V^{*}, h^{*}\right)$. A non-zero subspace $W \subset V^{*}$ is:

- hyperbolic iff $\operatorname{dim}(W \cap \mathcal{L})>1$, i.e. iff $W$ meets $\mathcal{L}$ along a sub-cone of the latter which has dimension at least two.

- degenerate iff $\operatorname{dim}(W \cap \mathcal{L})=1$, i.e. iff $W$ is tangent to $\mathcal{L}$ along a null line

- non-degenerate iff $W \cap \mathcal{L}=\{0\}$, in which case $W$ is spacelike (i.e. positive definite).

A degenerate subspace $W$ of $V^{*}$ contains no timelike vectors and the set of its null vectors coincides with the kernel $\mathrm{K}_{h}(W) \stackrel{\text { def. }}{=} \operatorname{ker}\left(h_{W}^{*}\right)$; accordingly, $W$ decomposes as:

$$
W=\mathrm{K}_{h}(W) \oplus U,
$$

where $\mathrm{K}_{h}(W)=\operatorname{ker}\left(h_{W}^{*}\right)$ coincides with the unique null line contained in $W$ and $U$ is a spacelike subspace of $V^{*}$ which is orthogonal to $\mathrm{K}_{h}(W)$. In particular, we have $W \subset$ $\mathrm{K}_{h}(W)^{\perp}$. For example, a 2-plane $\Pi \subset V^{*}$ can be spacelike, hyperbolic or degenerate, 
according to whether $h_{\Pi}^{*}$ is positive-definite, non-degenerate of signature $(1,1)$ or degenerate. In the latter case, $\Pi$ is called a parabolic 2-plane.

Definition A.2 A complete flag $0 \subset W_{(1)} \subset W_{(2)} \subset W_{(3)} \subset V^{*}$ is called degenerate if $W_{(i)}$ is a degenerate subspace of $\left(V^{*}, h^{*}\right)$ for each $i=1,2,3$.

Notice that a complete flag $0 \subset W_{(1)} \subset W_{(2)} \subset W_{(3)} \subset V^{*}$ is determined by the increasing sequence of vector spaces $W_{\bullet} \stackrel{\text { def. }}{=}\left(W_{(1)}, W_{(2)}, W_{(3)}\right)$. Such a flag is degenerate iff $W_{(2)}$ and $W_{(3)}$ are tangent to the light cone of $\left(V^{*}, h^{*}\right)$ along the line $W_{(1)}$ (whence $W_{(1)}$ is a null line).

Proposition A.3 There exists a natural bijection between the set of parabolic 2-planes and the set of degenerate complete flags in $\left(W^{*}, h^{*}\right)$. This associates to each parabolic 2-plane $\Pi \subset V^{*}$ the unique degenerate complete flag $0 \subset W_{(1)} \subset W_{(2)} \subset W_{(3)} \subset V^{*}$ with $W_{(2)}=\Pi$, which is given by:

$$
W_{(1)}=K_{h}(\Pi), \quad W_{(2)}=\Pi, \quad W_{(3)}=K_{h}(\Pi)^{\perp} .
$$

Proof Let $0 \subset W_{(1)} \subset W_{(2)} \subset W_{(3)} \subset V^{*}$ be a degenerate complete flag. Then the 2plane $\Pi \stackrel{\text { def. }}{=} W_{(2)}$ is degenerate, i.e. parabolic. Since the subspace $W_{(1)}$ of $W_{(2)}=\Pi$ is degenerate and one-dimensional, it is a null line and hence coincides with $\mathrm{K}_{h}(\Pi)$. Since $W_{(3)}$ is degenerate, the one-dimensional subspace $\mathrm{K}_{h}\left(W_{(3)}\right)$ is the unique null line contained in $W_{(3)}$ and hence coincides with $W_{(1)}$. We thus have $W_{(3)} \subset W_{(1)}^{\perp}$. This inclusion is an equality because $\operatorname{dim} W_{(1)}^{\perp}=\operatorname{dim} V^{*}-1=3=\operatorname{dim} W_{(3)}$. Hence any degenerate complete flag has the form (101) for a unique parabolic 2-plane $\Pi$, namely $\Pi=W_{(2)}$. It is clear that the correspondence thus defined is a bijection.

Definition A.4 A co-oriented degenerate complete flag in $\left(V^{*}, h^{*}\right)$ is a pair $\left(W_{\bullet}, L\right)$, where $W_{\bullet}=\left(W_{(1)}, W_{(2)}, W_{(3)}\right)$ is a degenerate complete flag in $\left(V^{*}, h^{*}\right)$ and $L$ is a co-orientation of the parabolic two-plane $W_{(2)}$.

Corollary 3.36 and Proposition A.3 imply the following reformulation of Theorem 3.34:

Theorem A.5 There exists a natural bijection between $\mathbb{P}(\Sigma)$ and the set of all co-oriented degenerate complete flags in $\left(V^{*}, h^{*}\right)$.

\section{Appendix B: Heterotic supergravity in four Lorentzian dimensions}

Let $G$ be a compact semisimple real Lie group whose Lie algebra we denote by $\mathfrak{g}$ and whose adjoint representation we denote by $\operatorname{Ad}_{G}: G \rightarrow \mathrm{GL}(\mathfrak{g})$. Let $\mathfrak{g}=\mathfrak{g}_{1} \oplus \cdots \oplus \mathfrak{g}_{k}$ be the decomposition of $\mathfrak{g}$ into simple Lie algebras. Then any $\mathrm{Ad}_{G}$-invariant non-degenerate symmetric pairing $\mathfrak{c}$ on $\mathfrak{g}$ can be written as:

$$
\mathfrak{c}=c_{1} B_{1} \oplus \cdots \oplus c_{k} B_{k}
$$

where $B_{j}$ is the Killing form of $\mathfrak{g}_{j}$ and $c_{j}$ are non-zero constants.

Definition B.1 A four-dimensional heterotic datum of type $G$ on $M$ is a triplet $(M, P, \mathfrak{c})$, where $M$ is an oriented open four-manifold, $P$ is a principal bundle over $M$ and $\mathfrak{c}$ is a non-degenerate symmetric and $\mathrm{Ad}_{G}$-invariant bilinear pairing on $\mathfrak{g}$. 
Let $(M, P, \mathfrak{c})$ be a four-dimensional heterotic datum. Let $\mathfrak{g}_{P} \stackrel{\text { def. }}{=} P \times \operatorname{Ad}_{G} \mathfrak{g}$ be the adjoint bundle of Lie algebras of $P$ and $\mathcal{A}_{P}$ be the affine space of connections on $P$. For any connection $A \in \mathcal{A}_{P}$, let $F_{A} \in \Omega^{2}\left(M, \mathfrak{g}_{P}\right)$ denote the curvature form of $A$. Let $\mathfrak{c}_{P}$ be the pairing induced by $\mathfrak{c}$ on the adjoint bundle $\mathfrak{g}_{P}$. Since $\mathfrak{c}$ is $\operatorname{Ad}_{G}$-invariant, the latter can be viewed as a morphism of vector bundles:

$$
\mathfrak{c}_{P}: \mathfrak{g}_{P} \otimes \mathfrak{g}_{P} \rightarrow \mathbb{R}_{M}
$$

where $\mathbb{R}_{M}$ is the trivial real line bundle on $M$. Let $\Lambda\left(M, \mathfrak{g}_{P}\right) \stackrel{\text { def. }}{=} \Lambda(M) \otimes \mathfrak{g}_{P}$ and consider the vector bundle morphism:

$$
\mathfrak{c}(-\wedge-) \stackrel{\text { def. }}{=} \wedge \otimes \mathfrak{c}: \Lambda\left(M, \mathfrak{g}_{P}\right) \otimes \Lambda\left(M, \mathfrak{g}_{P}\right) \rightarrow \Lambda(M),
$$

where $\wedge: \Lambda(M) \otimes \Lambda(M) \rightarrow \Lambda(M)$ is the wedge product. Let $\operatorname{Met}_{3,1}(M)$ be the set of metrics of signature $(3,1)$ defined on $M$. Let:

$\langle,\rangle_{g}: \Lambda(M) \times \Lambda(M) \rightarrow \mathbb{R}_{M}$ and $\langle,\rangle_{g, \mathfrak{c}}=\langle,\rangle_{g} \otimes \mathfrak{c}_{P}: \Lambda\left(M, \mathfrak{g}_{P}\right) \times\left(\Lambda\left(M, \mathfrak{g}_{P}\right) \rightarrow \mathbb{R}_{M}\right.$ be the metrics induced by $g \in \operatorname{Met}_{3,1}(M)$ and $\mathfrak{c}_{P}$ on the vector bundles $\Lambda(M)$ and $\Lambda\left(M, \mathfrak{g}_{P}\right)$. Consider the symmetric bilinear maps corresponding to the vector bundle morphisms:

$$
\begin{aligned}
& \circ: \Lambda(M) \otimes \Lambda(M) \rightarrow T^{*} M \otimes T^{*} M \text { and } \\
& \mathfrak{c}(-\circ-): \Lambda\left(M, \mathfrak{g}_{P}\right) \otimes \Lambda\left(M, \mathfrak{g}_{P}\right) \rightarrow T^{*} M \otimes T^{*} M
\end{aligned}
$$

whose action on global sections is given by:

$$
\begin{gathered}
(\omega \circ \eta)(X, Y) \stackrel{\text { def. }}{=}\left\langle\iota_{X} \omega, \iota_{Y} \eta\right\rangle_{g} \quad \forall \omega, \eta \in \Omega(M) \forall X, Y \in \mathfrak{X}(M) \\
\mathfrak{c}(\alpha \circ \beta)(X, Y) \stackrel{\text { def. }}{=}\left\langle\iota_{X} \alpha, \iota_{Y} \beta\right\rangle_{g, \mathfrak{c}} \quad \forall \alpha, \beta \in \Omega\left(M, \mathfrak{g}_{P}\right) \quad \forall X, Y \in \mathfrak{X}(M) .
\end{gathered}
$$

For a three-form $H \in \Omega^{3}(M)$ and the curvature $F_{A} \in \Omega^{2}\left(\mathfrak{g}_{P}\right)$ of a connection $A \in \mathcal{A}_{P}$, we have:

$$
\begin{aligned}
(H \circ H)(X, Y) & =\left\langle\iota_{X} H, \iota_{Y} H\right\rangle_{g} \quad \forall X, Y \in \mathfrak{X}(M), \\
\mathfrak{c}\left(F_{A} \circ F_{A}\right)(X, Y) & =\left\langle\iota_{X} F_{A}, \iota_{Y} F_{A}\right\rangle_{g, \mathfrak{c}} \quad \forall X, Y \in \mathfrak{X}(M) .
\end{aligned}
$$

Remark B.2 Pick a nonempty open subset $U \subset M$ which supports local coordinates $\left\{x^{i}\right\}_{i=1, \ldots, 4}$ of $M$ as well as a local $g$-orthonormal frame $\left\{e_{i}\right\}_{i=1, \ldots, 4}$ of $T M$ and a c-orthogonal local frame $\left\{T_{a}\right\}_{a=1, \ldots, \mathrm{rk} g}$ of $\mathfrak{g}_{P}$ such that $\mathfrak{c}\left(T_{a}, T_{b}\right)=\delta_{a b} \varepsilon_{a}$, where $\varepsilon_{a} \in\{-1,1\}$. Then the following relations hold on $U$ :

$$
\begin{aligned}
& \mathfrak{c}\left(F_{A} \wedge F_{A}\right)=\varepsilon_{a} F_{A}^{a} \wedge F_{A}^{a}, \quad \mathfrak{c}\left(F_{A} \circ F_{A}\right)_{i j}=\varepsilon_{a}\left(F_{A}^{a}\right)_{i m}\left(F_{A}^{a}\right)_{j k} g^{m k}, \\
& (H \circ H)_{i j}=H_{i l m} H_{j}^{l m},
\end{aligned}
$$

where $F_{A}=F_{A}^{a} T_{a}$ and we use Einstein summation over $i, j, l, m=1, \ldots, 4$ and over $a=1, \ldots \mathrm{rk} \mathfrak{g}$.

Let $\Omega_{\mathrm{cl}}^{1}(M)$ be the space of closed one-forms defined on $M$.

Definition B.3 A bosonic heterotic configuration of $(M, P, \mathfrak{c})$ is a quadruplet $(g, \varphi, H, A) \in$ $\operatorname{Met}_{3,1}(M) \times \Omega_{\mathrm{cl}}^{1}(M) \times \Omega^{3}(M) \times \mathcal{A}_{P}$ which satisfies the modified Bianchi identity:

$$
\mathrm{d} H=\mathfrak{c}\left(F_{A} \wedge F_{A}\right) .
$$


The one-form $\varphi \in \Omega_{\mathrm{cl}}^{1}(M)$ is called the dilatonic one-form of the bosonic heterotic configuration. On sufficiently small non-empty open subsets $U \subset M$, it can be written as $\varphi=\mathrm{d} \phi$, where the locally defined function $\phi \in \mathcal{C}^{\infty}(U)$ corresponds to the dilaton of the physics literature.

Definition B.4 A bosonic heterotic configuration $(g, \varphi, H, A)$ of $(M, P, \mathfrak{c})$ is called bosonic heterotic solution if it satisfies the equations of motion:

$$
\begin{aligned}
\operatorname{Ric}^{g}+\nabla^{g} \varphi-\frac{1}{4} H \circ H-\mathfrak{c}\left(F_{A} \circ F_{A}\right) & =0, \\
\mathrm{~d}^{*} H+\iota_{\varphi} H & =0, \\
\mathrm{~d}_{A} * F_{A}-\varphi \wedge * F_{A}+F_{A} \wedge * H & =0, \\
\mathrm{~d}^{*} \varphi+\langle\varphi, \varphi\rangle_{g}-\langle H, H\rangle_{g}-\left\langle F_{A}, F_{A}\right\rangle_{g, \mathfrak{c}} & =0 .
\end{aligned}
$$

Let $\operatorname{Conf}(M, P, \mathfrak{c})$ and $\operatorname{Sol}(M, P, \mathfrak{c}) \subset \operatorname{Conf}(M, P, \mathfrak{c})$ be the sets of bosonic configurations and solutions of the heterotic datum $(M, P, \mathfrak{c})$.

Remark B.5 If G is the trivial group, then $P$ is the unit principal bundle over $M$, which has total space $M$ and projection given by the identity $\operatorname{map~id}_{M}$. In this case, $\mathfrak{c}$ vanishes (as does any connection on $P$ ) and the set of bosonic configurations reduces to:

$$
\operatorname{Conf}_{0}(M)=\operatorname{Met}_{3,1}(M) \times \Omega_{\mathrm{cl}}^{1}(M) \times \Omega_{\mathrm{cl}}^{3}(M),
$$

since the modified Bianchi identity requires $\mathrm{d} H=0$. Moreover, the equations of motion reduce to:

$$
\begin{aligned}
& \operatorname{Ric}^{g}+\nabla^{g} \varphi-\frac{1}{4} H \circ H=0, \quad \mathrm{~d}^{*} H+\iota_{\varphi} H=0, \\
& \mathrm{~d}^{*} \varphi+\langle\varphi, \varphi\rangle_{g}-\langle H, H\rangle_{g}=0 .
\end{aligned}
$$

This particular case is known as NS-NS supergravity.

For any three-form $H$ on $M$, let $\nabla^{H}$ be the unique $g$-compatible connection on $T M$ with totally skew-symmetric torsion given by $T=H^{\sharp}$, where $H^{\sharp} \in \Gamma\left(T^{*} M \otimes T^{*} M \otimes T M\right)$ is defined through:

$$
H^{\sharp}(X, Y)=\left(\iota_{Y} \iota_{X} H\right)^{\sharp} \in \mathfrak{X}(M) \quad \forall X, Y \in \mathfrak{X}(M) .
$$

Here $\sharp$ denotes raising of indices with respect to $g$. This connection is given by:

$$
\nabla^{H}=\nabla^{g}-\frac{1}{2} H^{\sharp} .
$$

Assume that $M$ admits strongly spinnable Lorentzian metrics, whose space we denote by $\operatorname{Met}_{3,1}^{\mathrm{ss}}(M)$. We shall assume for simplicity that $H^{1}\left(M, \mathbb{Z}_{2}\right)=0$, although this can be relaxed. For any $g \in \operatorname{Met}_{3,1}^{\mathrm{ss}}(M)$, let $\left(S_{g}, \Gamma_{g}\right)$ be a spinor bundle on $(M, g)$. The assumption $H^{1}\left(M, \mathbb{Z}_{2}\right)=0$ implies that this spinor bundle is unique up to isomorphism.

Definition B.6 A bosonic heterotic configuration $(g, \varphi, H, A) \in \operatorname{Conf}(M, P, \mathfrak{c})$ is called supersymmetric if $g \in \operatorname{Met}_{3,1}^{\mathrm{ss}}(M)$ and there exists a nontrivial spinor $\epsilon \in \Gamma\left(S_{g}\right)$ which satisfies the Killing spinor equations:

$$
\hat{\nabla}^{H} \epsilon=0, \varphi \cdot \epsilon=H \cdot \epsilon=0, \quad F_{A} \cdot \epsilon=0 .
$$

A similar formulation can be given for heterotic supergravity on a ten-dimensional open manifold. 


\section{References}

1. Agricola, I., Friedrich, T.: The SRNI lectures on non-integrable geometries with torsion. Arch. Math. 42, 5-84 (2006)

2. Agricola, I., Friedrich, T.: 3-Sasakian manifolds in dimension seven, their spinors and -structures. J. Geom. Phys. 60(2), 326-332 (2010)

3. Agricola, I., Höll, J.: Cones of G manifolds and Killing spinors with skew torsion. Ann. Mat. Pura Appl. 194(3), 673-718 (2015)

4. Alekseevsky, D.V., Cortés, V.: Classification of N-(super)-extended Poincaré algebras and bilinear invariants of the spinor representation of $\operatorname{Spin}(p, q)$. Commun. Math. Phys. 183(3), 477-510 (1997)

5. Alekseevsky, D.V., Cortés, V., Devchand, C., Proyen, A.V.: Polyvector super-Poincaré algebras. Commun. Math. Phys. 253(2), 385-422 (2005)

6. Alonso-Alberca, N., Lozano-Tellechea, E., Ortin, T.: Geometric construction of Killing spinors and supersymmetry algebras in homogeneous space-times. Class. Quantum Gravity 19, 6009 (2002)

7. Babalic, E.M., Lazaroiu, C.I.: The landscape of G-structures in eight-manifold compactifications of Mtheory. JHEP 1511, 007 (2015)

8. Bär, C.: Real Killing spinors and holonomy. Commun. Math. Phys. 154, 509-521 (1993)

9. Bär, C., Gauduchon, P., Moroianu, A.: Generalized cylinders in semi-Riemannian and spin geometry. Math. Z. 249, 545-580 (2005)

10. Bellorin, J., Ortin, T.: All the supersymmetric configurations of $\mathrm{N}=4, \mathrm{~d}=4$ supergravity. Nucl. Phys. B 726, 171-209 (2005)

11. Bellorin, J., Ortin, T.: A note on simple applications of the Killing spinor identities. Phys. Lett. B 616, 118-124 (2005)

12. Berezin, F.A., Marinov, M.S.: Particle spin dynamics as the Grassmann variant of classical mechanics. Ann. Phys. 104, 336-362 (1977)

13. Bergshoeff, E.A., Kallosh, R., Ortin, T.: Supersymmetric string waves. Phys. Rev. D 47, 5444 (1993)

14. Bernal, A.N., Sanchez, M.: On Smooth Cauchy hypersurfaces and Geroch's splitting theorem. Commun. Math. Phys. 243, 461-470 (2003)

15. Blaine Lawson, H., Michelsohn, M.-L.: Spin geometry. Princeton Mathematical Series, vol. 38 (1990)

16. Bohle, C.: Killing spinors on Lorentzian manifolds. J. Geom. Phys. 45(3-4), 285-308 (2003)

17. Brozos-Vázquez, M., García-Río, E., Gilkey, P., Nikcević, S., Vázquez-Lorenzo, R.: The geometry of Walker manifolds. Synth. Lect. Math. Stat. 2, 1 (2009)

18. Bryant, R.L.: Pseudo-Riemannian metrics with parallel spinor fields and vanishing Ricci tensor. Sémin. Congr. 4(53), 53-94 (2000). Soc. Math. France, Paris

19. Caldarelli, M.M., Klemm, D.: All supersymmetric solutions of $\mathrm{N}=2, \mathrm{D}=4$ gauged supergravity. JHEP 0309, 019 (2003)

20. Chevalley, C.: The Algebraic Theory of Spinors. Columbia University Press, New York (1954)

21. Chevalley, C.: The construction and study of certain important algebras, Math. Soc. Japan 1955. In: Collected Works, vol. 2. Springer, Berlin (1997)

22. Coimbra, A., Strickland-Constable, C.: Supersymmetric backgrounds, the Killing superalgebra, and generalised special holonomy. JHEP 1611, 063 (2016)

23. Conti, D., Salamon, S.: Generalized Killing spinors in dimension 5. Trans. Am. Math. Soc. 359(11), 5319-5343 (2007)

24. Duggal, K.L., Sahin, B.: Differential Geometry of Lightlike Submanifolds, Frontiers in Mathematics. Birkhäuser (2018)

25. Dunajski, M.: Anti-self-dual four-manifolds with a parallel real spinor. Proc. Math. Phys. Eng. Sci. 458(2002), 1205-1222 (2021)

26. Figueroa-O'Farrill, J.M.: The Homogeneity conjecture for supergravity backgrounds. J. Phys. Conf. Ser. 175, 012002 (2009)

27. Figueroa-O'Farrill, J.M., Papadopoulos, G.: Maximally supersymmetric solutions of ten-dimensional and eleven-dimensional supergravities. JHEP 0303, 048 (2003)

28. Freedman, D.Z., Van Proeyen, A.: Supergravity, Cambridge Monographs on Mathematical Physics, Cambridge (2012)

29. Friedrich, T.: On the spinor representation of surfaces in Euclidean 3-space. J. Geom. Phys. 28(1-2), 143-157 (1998)

30. Friedrich, T.: Dirac operators in Riemannian geometry, Graduate Studies in Mathematics, vol. 25. AMS, Providence (2000)

31. Friedrich, T., Ivanov, S.: Parallel spinors and connections with skew symmetric torsion in string theory. Asian J. Math. 6, 303-336 (2002) 
32. Friedrich, T., Kath, I.: 7-Dimensional compact Riemannian manifolds with Killing spinors. Commun. Math. Phys. 133, 543-561 (1990)

33. Friedrich, T., Kim, E.C.: The Einstein-Dirac equation on Riemannian spin manifolds. J. Geom. Phys. 33(1-2), 128-172 (2000)

34. Friedrich, T., Kim, E.C.: Some remarks on the Hijazi inequality and generalizations of the Killing equation for spinors. J. Geom. Phys. 37(1-2), 1-14 (2001)

35. Friedrich, T., Trautman, A.: Spin spaces, Lipschitz groups and spinor bundles. Ann. Glob. Anal. Geom. 18(3-4), 221-240 (2000)

36. Galaev, A.S.: Examples of Einstein spacetimes with recurrent null vector fields. Class. Quantum Gravity 28, $175022(2011)$

37. Galaev, A.S., Leistner, T.: On the local structure of Lorentzian Einstein manifolds with parallel distribution of null lines. Class. Quantum Gravity 27, 225003 (2010)

38. Garcia-Fernandez, M.: Lectures on the Strominger system. Travaux Math. XXIV, 7-61 (2016)

39. Garcia-Fernandez, M., Rubio, R., Tipler, C.: Infinitesimal moduli for the Strominger system and Killing spinors in generalized geometry. Math. Ann. 369(1-2), 539-595 (2017)

40. Gauntlett, J.P., Gutowski, J.B.: All supersymmetric solutions of minimal gauged supergravity in fivedimensions. Phys. Rev. D 68, 105009 (2003) [Erratum, Phys. Rev. D 70, 089901 (2004)]

41. Gauntlett, J.P., Pakis, S.: The geometry of D = 11 Killing spinors. JHEP 0304, 039 (2003)

42. Gauntlett, J.P., Gutowski, J.B., Hull, C.M., Pakis, S., Reall, H.S.: All supersymmetric solutions of minimal supergravity in five- dimensions. Class. Quantum Gravity 20, 4587-4634 (2003)

43. Gauntlett, J.P., Gutowski, J.B., Pakis, S.: The geometry of D = 11 null Killing spinors. JHEP 0312, 049 (2003)

44. Gibbons, G.W.: Anti-de-Sitter spacetime and its uses. In: Cotsakis, S., Gibbons, G.W. (eds.) Mathematical and Quantum Aspects of Relativity and Cosmology, Lecture Notes in Physics, vol. 537. Springer (2000)

45. Gibbons, G.W., Hull, C.M.: A Bogomolny bound for general relativity and solitons in N=2 supergravity. Phys. Lett. B 109, 190-194 (1982)

46. Gibbons, G.W., Ruback, P.J.: Classical gravitons and their stability in higher dimensions. Phys. Lett. B 171, 390-395 (1986)

47. Graf, W.: Differential forms as spinors. Ann. I.H.P. Phys. Théor. 29(1), 85-109 (1978)

48. Gran, U., Lohrmann, P., Papadopoulos, G.: The spinorial geometry of supersymmetric heterotic string backgrounds. JHEP 0602,063 (2006)

49. Gran, U., Papadopoulos, G., Roest, D.: Supersymmetric heterotic string backgrounds. Phys. Lett. B 656, 119-126 (2007)

50. Gran, U., Papadopoulos, G., Roest, D., Sloane, P.: Geometry of all supersymmetric type I backgrounds. JHEP 0708, 074 (2007)

51. Gran, U., Gutowski, J., Papadopoulos, G.: Classification, geometry and applications of supersymmetric backgrounds. Phys. Rep. 794, 1 (2019)

52. Graña, M.: Flux compactifications in string theory: a comprehensive review. Phys. Rep. 423, 91-158 (2006)

53. Graña, M., Minasian, R., Petrini, M., Tomasiello, A.: Supersymmetric backgrounds from generalized Calabi-Yau manifolds. JHEP 0408, 046 (2004)

54. Graña, M., Shahbazi, C.S., Zambon, M.: Spin(7)-manifolds in compactifications to four dimensions. JHEP 1411, 046 (2014)

55. Grunewald, R.: Six-dimensional Riemannian manifolds with a real Killing spinor. Ann. Glob. Anal. Geom. 8, 43-59 (1990)

56. Harvey, F.R.: Spinors and Calibrations, Perspectives in Mathematics (1990)

57. Herrera, R., Herrera, H.: $\operatorname{Spin}^{q}$ manifolds admitting parallel and Killing spinors. J. Geom. Phys. 57(7), $1525-1539$ (2007)

58. Hitchin, N.: Harmonic spinors. Adv. Math. 14(1), 1-55 (1974)

59. Ikemakhen, A.: Parallel spinors on Lorentzian Spin ${ }^{c}$ manifolds. Differ. Geom. Appl. 25(3), 299-308 (2007)

60. Ivanov, S.: Connections with torsion, parallel spinors and geometry of Spin(7)-manifolds. Math. Res. Lett. 2-4, 171-186 (2004)

61. Kähler, E.: Der innere Differentialkalkül. Rend. Mat. 3-4(21), 425-523 (1960)

62. Karoubi, M.: Algebres de Clifford et K-theorie. Ann. Sci. Ec. Norm. Sup. 1(2), 161-270 (1968)

63. Lazaroiu, C.I., Babalic, E.M.: Geometric algebra techniques in flux compactifications II. JHEP 1306, 054 (2013)

64. Lazaroiu, C.I., Babalic, E.M.: Foliated eight-manifolds for M-theory compactification. JHEP 1501, 140 (2015)

65. Lazaroiu, C.I., Babalic, E.M.: Singular foliations for M-theory compactification. JHEP 03, 116 (2015) 
66. Lazaroiu, C.I., Babalic, E.M.: Geometric algebra techniques in flux compactifications. Adv. High Energy Phys. 2016, 7292534 (2016)

67. Lazaroiu, C.I., Shahbazi, C.: Complex Lipschitz structures and bundles of complex Clifford modules. Differ. Geom. Appl. 61, 147-169 (2018)

68. Lazaroiu, C.I., Shahbazi, C.: On the spin geometry of supergravity and string theory, Geometric Methods in Physics XXXVI, pp. 229-235, Trends in Mathematics. Birkhauser (2019)

69. Lazaroiu, C.I., Shahbazi, C.: Real pinor bundles and real Lipschitz structures. Asian J. Math. (to appear)

70. Lazaroiu, C.I., Babalic, E.M., Coman, I.A.: The geometric algebra of Fierz identities in various dimensions and signatures. JHEP 09, 156 (2013)

71. Lazaroiu, C.I., Babalic, E.M., Coman, I.A.: The geometric algebra of supersymmetric backgrounds. String-Math 2012—Proc. Symp. Pure Math., vol. 90. AMS, pp. 227-237 (2015)

72. Leistner, T.: Lorentzian manifolds with special holonomy and parallel spinors, Proc. 21st Winter School Geometry and Physics, Rend. Cir. Mat. Palermo, Ser. II Suppl. 69 (2002)

73. Leitner, F.: Imaginary Killing spinors in Lorentzian geometry. J. Math. Phys. 44, 4795 (2003)

74. Lu, H., Pope, C.N., Rahmfeld, J.: A construction of Killing spinors on $S^{n}$. J. Math. Phys. 40, 4518-4526 (1999)

75. Meinrenken, E.: Clifford algebras and Lie theory, Ergebnisse der Mathematik und ihrer Grenzgebiete. 3. Folge, Springer (2013)

76. Moroianu, A.: Parallel and Killing spinors on $\operatorname{spin}^{c}$ manifolds. Commun. Math. Phys. 187, 417-427 (1997)

77. Moroianu, A.: Parallel spinors and holonomy groups. J. Math. Phys. 41(4), 2395-2402 (2002)

78. Moroianu, A., Semmelmann, U.: Generalized Killing spinors on Einstein manifolds. Int. J. Math. 25(4), 1450033 (2014)

79. Moroianu, A., Semmelmann, U.: Generalized Killing spinors and Lagrangian graphs. Differ. Geom. Appl. 37, 141-151 (2014)

80. Moroianu, A., Semmelmann, U.: Generalized Killing spinors on spheres. Ann. Glob. Anal. Geom. 46, 129-143 (2014)

81. Ortín, T.: Gravity and Strings, 2nd edn, Cambridge Monographs on Mathematical Physics, Cambridge (2015)

82. Papadopoulos, G.: Heterotic supersymmetric backgrounds with compact holonomy revisited. Class. Quantum Gravity 27, 125008 (2010)

83. Riesz, M.: Clifford algebras and spinors. In: Bolinder, E.F., Lounesto, P. (eds.) Fundamental Theories of Physics 54. Springer, Berlin (1993)

84. Tod, K.P.: All metrics admitting supercovariantly constant spinors. Phys. Lett. B 121, 241 (1983)

85. Tod, K.P.: More on supercovariantly constant spinors. Class. Quantum Gravity 12, 1801-1820 (1995)

86. Voronov, F.F.: Quantization on supermanifolds and an analytic proof of the Atiyah-Singer index theorem. J. Sov. Math. 64(4), 993-1069 (1993)

87. Walker, A.G.: On parallel fields of partially null vector spaces. Q. J. Math. 20(1), 135-145 (1949)

88. Wang, M.Y.: Parallel spinors and parallel forms. Ann. Glob. Anal. Geom. 7(1), 59-68 (1989)

Publisher's Note Springer Nature remains neutral with regard to jurisdictional claims in published maps and institutional affiliations. 